\title{
CATEGORICAL ALGEBRA
}

\section{SAUNDERS MACLANE}

1. Introduction. Category, functor, and natural transformation of functors are notions of great generality-and consequent simplicity. They apply to many different parts of mathematics. Now 22 years old, they have recently attracted especially active interest in many quarters. This interest is probably a reflection of the very rapid current proliferation of mathematical ideas-a situation favoring and, indeed, almost requiring unifying notions such as those of category and functor.

This article, a revision of the notes used in the Colloquium Lectures of the American Mathematical Society for 1963, will summarize a number of the developments which use categories, with particular attention to the ubiquity of adjoint functors, the utility of abelian categories, a unified categorical treatment of types of algebras, relative homological algebra via adjoint functors, differential graded objects, and universal algebra via suitable "very small" categories. For some items of detail, I refer to my book Homology [77] and references in the style "Gertrude Stein [1929]" are to the bibliography there. Other references, in the usual style, are to the bibliography at the end, which is intended to cover recent literature of the subject in tolerable completeness.

Especial acknowledgments are due to S. Eilenberg, with whom I collaborated in the initial development of some of the ideas presented here. My own approach has also been much influenced by the stimulating ideas of a number of young mathematicians, especially Freyd, Harrison, Kelly, Lawvere, and Linton. My studies on this subject have been supported in part by a grant from the National Science Foundation, and more extensively over the years under several contracts and grants from the Air Force Office of Scientific Research; the preparation of the revision of this paper was supported in part by the National Science Foundation under grant NSF G-16428 to the American Mathematical Society.

\section{Chapter I. Functors and Adjoints}

2. Categories. Let $\mathrm{C}$ be a class of objects $A, B, C, \cdots$ together with a family of disjoint sets $\operatorname{hom}(A, B)$, one for each ordered pair $A, B$ of objects. Write $f: A \rightarrow B$ for $f \in \operatorname{hom}(A, B)$, and call $f$ a map or

Received by the editors October 6, 1964. 
a morphism of $\mathbf{C}$ with domain $A$ and codomain $B$. Assume a rule which assigns to each pair $f: A \rightarrow B, g: B \rightarrow C$ of morphisms a unique morphism $g f=g \circ f: A \rightarrow C$, called their composite, and note that this composite $g f$ is defined precisely when the range of $f$ is the domain of $g$. There are two axioms:

Associativity: If $f: A \rightarrow B, g: B \rightarrow C$, and $h: C \rightarrow D$, then $h(g f)=(h g) f$; Identity: To each object $B$ there exists a morphism $1_{B}: B \rightarrow B$ such that always

$$
1_{B} f=f, g 1_{B}=g, \text { for } f: A \rightarrow B \text { and } g: B \rightarrow C .
$$

Under these axioms, $\mathbf{C}$ is called a category. In a category $\mathbf{C}$ the set $\operatorname{hom}(A, B)$ of morphisms from $A$ to $B$ will be variously denoted as

$$
\operatorname{hom}(A, B)=\operatorname{hom}_{\mathrm{C}}(A, B)=\mathrm{C}(A, B)=\mathrm{C}\left(\begin{array}{l}
A \\
B
\end{array}\right) \text {. }
$$

Given the object $B$, the identity morphism $1_{B}$ is uniquely determined by the properties displayed above. Indeed, a category may be described completely in terms of its morphisms, ignoring the objects. Thus let $\mathbf{C}$ be a class of "morphisms" $f, g, h$ in which a composite $g f$ is sometimes defined. Call a morphism $u$ an identity of $\mathbf{C}$ if $u f=f$ whenever the composite $u f$ is defined and $g u=g$ whenever $g u$ is defined. The axioms then are:

(i) The product $h(g f)$ is defined if and only if the product $(h g) f$ is defined. When either is defined, they are equal. This triple product will be written as $h g f$;

(ii) The triple product $h g f$ is defined whenever both products $h g$ and $g f$ are defined;

(iii) For each morphism $f$ of $\mathbf{C}$ there exist identities $u$ and $u^{\prime}$ such that $u^{\prime} f$ and $f u$ are defined.

It is readily verified that this definition is equivalent to the preceding one and that the identity morphisms are precisely the morphisms $1_{B}$, which are in one-one correspondence $1_{B} \leftrightarrow B$ with the objects. A category with only one object (often called a monoid) is just the same thing as a semigroup with identity element.

In a category $\mathrm{C}$, a morphism $e: A \rightarrow B$ is invertible (or an equivalence) if there is a morphism $e^{\prime}: B \rightarrow A$ with $e^{\prime} e=1_{A}$ and $e e^{\prime}=1_{B}$. If $e^{\prime}$ exists, it is unique, and is written as $e^{\prime}=e^{-1}$. As usual, $\left(e_{1} e_{2}\right)^{-1}$ $=e_{2}^{-1} e_{1}^{-1}$ when $e_{1} e_{2}$ is defined and both $e_{1}$ and $e_{2}$ are invertible. Two objects $A$ and $B$ are equivalent in $\mathrm{C}$ if there is an invertible $e: A \rightarrow B$. A category in which every morphism is invertible is a (Brandt) groupoid; a groupoid with only one object is a group. 
A morphism $k: A \rightarrow B$ is monic in $\mathbf{C}$ if it is left cancellable. Dually, a morphism $u: A \rightarrow B$ is epic if it is right cancellable.

An object $T$ is terminal in $\mathbf{C}$ if to each object $A$ there is exactly one morphism $A \rightarrow T$. (This terminology is due to J. A. Zilber.) If $T$ is terminal, the only morphism $T \rightarrow T$ is the identity, and any two terminal objects in $\mathbf{C}$ are equivalent. Dually, an object $J$ in $\mathbf{C}$ is initial if to each object $A$ there is exactly one morphism $J \rightarrow A$.

Next, some examples:

The category Ens of sets has as objects all sets $S, T, \cdots$, as morphisms all functions from $S$ to $T$ with the usual composition ( $g f$ means first apply $f$, then $g$ ). In this category, the monics are the injections (the functions one-one into), and the epics are the surjections (functions onto). The empty set is initial, and any one-point set is a terminal object.

The category $\mathbf{G r}$ of groups has objects all (multiplicative) groups, morphisms all group homomorphisms. Clearly, the monic morphisms are the monomorphisms, while, with a more delicate argument, the epics are the usual epimorphisms. The group with one element is both initial and terminal in this category.

$\mathrm{Ab}$, the category of all abelian groups, has objects all (additive) abelian groups and morphisms all groups homomorphisms. Again, monics in $\mathrm{Ab}$ are monomorphisms and epics are group epimorphisms.

If $R$ is any ring, the category $R$-Mod has objects all left $R$-modules, morphisms all homomorphisms of such. There is a similar category of right $R$-modules, of $R$-bimodules, etc.

The category Top of topological spaces has as objects all topological spaces $X, Y, \cdots$ and as morphisms all continuous maps $f: X \rightarrow Y$. Again, monics are injections and epics, surjections. The one-point space is terminal, and the empty space is initial. Similarly, one may form the category of all Hausdorff spaces or of all compact Hausdorff spaces.

The category Htp has as objects all topological spaces $X, Y, \cdots$, while a morphism $\alpha: X \rightarrow Y$ is a homotopy class of continuous maps $f: X \rightarrow Y$; in other words, two homotopic maps $f \simeq g: X \rightarrow Y$ determine the same morphism from $X$ to $Y$. The composition of morphisms is the usual composition of homotopy classes of maps. In this category, the homotopy class of an injection need not be a monic, as one may see, for example, for the injection of a circle into a disc (as the bounding circle of that disc). This category $\mathrm{Htp}$, which arises naturally in homotopy theory, shows that a morphism in a category need not be the same thing as a function. There are a number of other categories which are useful in homotopy theory: For example, the categories of CW-complexes, of simplicial sets, and of Kan complexes. 
Ens* will denote the category of pointed sets. By a pointed set is meant a nonvoid set $P$ with a selected element, written $*$ or $*_{P}$, and called the "base point" of $P$. A map $f: P \rightarrow Q$ of pointed sets is a function on the set $P$ to the set $Q$ which carries base point to base point; i.e., which satisfies $f\left(*_{P}\right)=*_{Q}$. The pointed sets with these maps as morphisms constitute the category Ens $*$. In this category the set $\{*\}$ with just one point (the base point) is both an initial and a terminal object. A morphism $f$ is monic in Ens* if and only if it has a left inverse, epic if and only if it has a right inverse, and invertible if and only if it is both monic and epic.

Similarly, Top* denotes the category of pointed topological spaces: the objects are spaces $X$ with a designated base point *; the morphisms are continuous maps $f: X \rightarrow Y$ which send the base point of $X$ to that of $Y$. Again, $\mathrm{Htp}_{*}$ is the category with objects pointed spaces and morphisms homotopy classes of continuous base-point-preserving maps (where also the homotopies are to preserve base points). Both categories arise in homotopy theory, where the choice of a base point is always made in defining the fundamental group or higher homotopy groups of a space.

Another example: Let $P$ be any partly ordered set; that is, a set equipped with a binary relation $a \leqq b$ which is reflexive and transitive. Make $P$ into a category $\mathbf{P}$ with objects the elements of $P$, while the set $\operatorname{hom}_{\mathbf{P}}(a, b)$ is either empty or has exactly one element, the latter when $a \leqq b$. The composition rule is then forced. Any small category in which no set hom $(A, B)$ has more than one element arises from a partly ordered set in this way.

These various examples indicate that each type of mathematical system gives rise to a corresponding category, whose objects are the systems of that type and whose morphisms are the maps of such systems. Put differently, this approach suggests that whenever a new type of mathematical system is defined, one should simultaneously define the morphisms of that system.

A category has been described here as a "class" of objects (or as a class of morphisms). Here "class" is used in the sense of GödelBernays set theory: In that theory one may form the class of all sets or of all groups (where a group is regarded as a set with added structure, etc.). This avoids the difficulties otherwise attendant upon the "set of all sets." In particular, if the class of objects (and hence the class of all morphisms) of a category $\mathbf{C}$ is a set, we call $\mathbf{C}$ a small category. For certain further purposes, the treatment of categories as Gödel-Bernays classes does not allow enough flexibility; for example, one can speak of the category of all small categories but not of the category of all categories. These difficulties are outlined in MacLane 
[76]. Various types of solutions to these foundational difficulties have been proposed: The use of "locally small" categories [76], the use of a set theory with an ample supply of strongly inaccessible cardinal numbers; Grothendieck's use of "universe," with an axiom insuring that every set is contained in a universes (Gabriel [1962]); see also Sonner [95]. In closely related connections, Lawvere (unpublished) has considered axioms on the category of all sets and on the category of all small categories.

3. Constructions on categories. If $\mathbf{C}_{1}$ and $\mathbf{C}_{2}$ are categories, their product $\mathbf{C}_{1} \times \mathbf{C}_{2}$ is the category with objects the ordered pairs $\left(A_{1}, A_{2}\right)$ of objects $A_{i} \in \mathbf{C}_{i}$, while a morphism $\left(A_{1}, A_{2}\right) \rightarrow\left(B_{1}, B_{2}\right)$ is an ordered pair $\left(f_{1}, f_{2}\right)$ of morphisms $f_{i}: A_{i} \rightarrow B_{i}$, for $i=1,2$, with the evident composition. The product of any indexed family of categories is similarly defined.

Each category $\mathbf{C}$ determines a dual or opposite category $\mathrm{C}^{\circ}$. The objects of $\mathbf{C}^{\text {op }}$ are the objects of $\mathbf{C}$, while the morphisms $f^{*}: B \rightarrow A$ of $\mathbf{C}^{\mathrm{op}}$ are in one-one correspondence with the morphisms $f: A \rightarrow B$ of $\mathbf{C}$, but note that the direction is reversed. The composite $f^{*} g^{*}=(g f)^{*}$ is defined in $\mathbf{C}^{\text {op }}$ exactly when $g f$ is defined in C. Hence the codomain of $f^{*}$ is the domain of $f$, while $f^{*}$ is monic if and only if $f$ is epic, etc. Passage from $\mathbf{C}$ to $\mathbf{C}^{\text {op }}$ yields a categorical duality. Alternatively, the axioms for categories are self-dual. Hence there is a meta-mathematical duality: Any demonstrable theorem about categories remains demonstrable if the order of composition is reversed. This reversal amounts to reversing all arrows, interchanging "domain" with "codomain," "monic" with "epic," and "terminal" with "initial." This type of duality, cf. [75], has been extensively studied in topological cases by Eckmann-Hilton. More recently, there has been an explicit formulation of a notion of dual functor (Fuks [35], [36]; Fuks-Švarc [37], Mitjagin-Švarc [81], Švarc [101]), with existence theorems for such functors (Linton [71]).

For any category $\mathbf{C}$, the category $\operatorname{Morph}(\mathbf{C})$ has as objects the morphisms $f: A \rightarrow B$ of $\mathbf{C}$ and as morphisms $m: f \rightarrow f^{\prime}$ the pairs $m=(a, b)$ of morphisms $a: A \rightarrow A^{\prime}$ and $b: B \rightarrow B^{\prime}$ of $\mathrm{C}$ such that the square
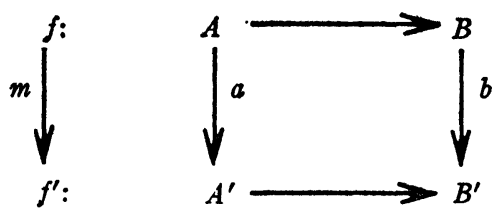

commutes. The composition of two such morphisms is evident. (Paste the first square on top of the second and erase the junction.) Actually, 
these squares can be regarded as the morphisms of a different category (paste the first square to the left of the second, and erase $b$ ); the squares are thus morphisms of two categories, suggesting Ehresmann's extensive investigation of "double categories" [23].

The category $\operatorname{Morph}(\mathbf{C})$ is but one example of many similar constructions. For instance, one may consider the category whose objects are all commutative squares; the morphisms are then evidently quadruples of morphisms of $\mathbf{C}$ which form, with the two given squares, an appropriately commutative cube.

For a fixed object $A$ in the category $\mathrm{C}$ we may form the category $\mathrm{C} / A$ of "morphisms with range $A$." An object in $\mathrm{C} / A$ is a morphism $h: H \rightarrow A$ (for any $H$ ); if $h$ and $h^{\prime}: H^{\prime} \rightarrow A$ are two such objects, a morphism $f: h \rightarrow h^{\prime}$ of $\mathbf{C} / A$ is a morphism $f: H \rightarrow H^{\prime}$ of $\mathbf{C}$ such that $h^{\prime} f=h$; the composition of morphisms of $\mathbf{C} / A$ is evident (that of $\mathbf{C}$ ). This construction has been used extensively by Grothendieck, who calls $\mathbf{C} / A$ the category of objects above $A$.

Similarly, there is a category $\mathrm{C} / \mathrm{m} A$ whose objects $k: K \rightarrow A$ are the monics of $\mathbf{C}$ with range $A$. A subobject of $A$ may be defined to be an equivalence class of objects in this category (cf. Homology, XII, §2). A dual procedure defines quotient objects. Under suitable assumptions on the category one may then construct the lattice of subobjects (or, dually, of quotient objects) of an object $A$. In this fashion, lattice theory, which started from similar general considerations of algebraic systems, appears as a suitable part of the study of categories.

4. Functors. A functor is a map of categories. More explicitly, a (covariant) functor $F: \mathbf{B} \rightarrow \mathbf{C}$ on $\mathbf{B}$ to $\mathbf{C}$ consists of an object function $F$ and a mapping function, also written $F$. The object function assigns to each object $B$ of $\mathbf{B}$ an object $F(B)$ of $\mathbf{C}$; the mapping function assigns to each morphism $f: B \rightarrow B^{\prime}$ of $\mathbf{C}$ a morphism $F(f): F(B)$ $\rightarrow F\left(B^{\prime}\right)$ of $\mathbf{B}$ in such a way that

$$
F\left(1_{B}\right)=1_{F(B)}, \quad F(g f)=(F g)(F f),
$$

the latter whenever the composite $g f$ is defined. The composite of two functors $G: \mathbf{C} \rightarrow \mathbf{D}$ and $F: \mathbf{B} \rightarrow \mathbf{C}$ is a functor $G \circ F: \mathbf{B} \rightarrow \mathbf{D}$. Under this composition, the functors are the morphisms of a category; more exactly, of Cat, the category of all small categories.

Note some examples of functors. First, let $F(S)$ be the free group generated by the set $S$. Since each function $f: S \rightarrow S^{\prime}$ can be extended to a unique group homomorphism $F(f): F(S) \rightarrow F\left(S^{\prime}\right)$, this yields a functor $F:$ Ens $\rightarrow$ Gr on the category of sets to that of groups. Again, the function which assigns to each Lie group its associated Lie alge- 
bra is (the object function of) a functor on the category of Lie groups to that of Lie algebras; much of the elementary theory of Lie groups can be regarded as the development of properties of this functor. In topology, the construction of the fundamental group $\pi_{1}(X)$ of a space $X$ yields a functor $\pi_{1}$ : Top $\rightarrow \rightarrow G r$; indeed, since maps of spaces homotopic (relative to the base point) induce the same homomorphism on the fundamental groups of these spaces, we may regard $\pi_{1}$ as a functor defined on the category $\mathrm{Htp}_{*}$ discussed above, in which the morphisms are homotopy classes of continuous maps. Similarly, for $n>1$, each higher homotopy group yields a functor $\pi_{n}: H t p_{*} \rightarrow \mathrm{Ab}$. The cohomology groups (singular, Čech, or Alexander-Spanier) of a space yield functors $H^{n}$ : Top $\rightarrow \mathrm{Ab}$; the homotopy axiom for these cohomology groups states that homotopic maps yield the same group homomorphism, and hence each $H^{n}$ is, in reality, a functor $\mathrm{Htp} \rightarrow \mathrm{Ab}$. The standard axiomatic homology or cohomology theory is a formulation of the properties of these functors, and would hardly have been possible without the language of categories and functors. The more recent "extraordinary" cohomology theories, such as $K$-theory, are also instances of functors.

On any product category $\mathbf{C}_{1} \times \mathbf{C}_{2}$, there are two "projection" functors

$$
P_{1}: \mathrm{C}_{1} \times \mathrm{C}_{2} \rightarrow \mathrm{C}_{1}, \quad P_{2}: \mathrm{C}_{1} \times \mathrm{C}_{2} \rightarrow \mathrm{C}_{2}
$$

defined on ordered pairs by $P_{i}\left(C_{1}, C_{2}\right)=C_{i}$ and $P_{i}\left(f_{1}, f_{2}\right)=f_{i}$, for $i=1,2$. Moreover, to any pair of functors $F_{i}: \mathbf{B} \rightarrow \mathbf{C}_{i}$ with a common domain category $\mathbf{B}$ there is a unique functor $G: \mathbf{B} \rightarrow \mathbf{C}_{1} \times \mathbf{C}_{2}$ with $P_{1} G=F_{1}$ and $P_{2} G=F_{2}$. The product category $\mathbf{C}_{1} \times \mathbf{C}_{2}$ with its projections (2) is determined up to isomorphism by this property. (An isomorphism of categories $\mathbf{C}, \mathbf{C}^{\prime}$ is a functor $J: \mathbf{C} \rightarrow \mathbf{C}^{\prime}$ whose object function and mapping function are both bijections.)

Given two functors $F, G: \mathbf{B} \rightarrow \mathbf{C}$, a natural transformation $t: F \rightarrow G$ is a function $t$ which assigns to each object $B$ of $\mathbf{B}$ a morphism $t(B)$ : $F(B) \rightarrow G(B)$ of $\mathbf{C}$ in such a way that every morphism $f: B \rightarrow B^{\prime}$ yields a commutative diagram

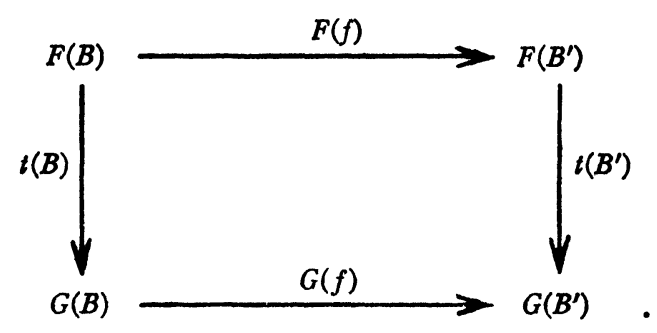


If, in addition, each $t(B)$ is invertible in $\mathrm{C}$, call $t: F \rightarrow G$ a natural isomorphism or a natural equivalence, and write $t: F \cong G$. Note that the inverses $t(B)^{-1}$ then constitute a natural $t^{-1}: G \cong F$. For example, let Abf be the category of all finite abelian groups and $I: \mathrm{Abf} \rightarrow \mathrm{Abf}$ the identity functor. Let $D(G)$ be the character group of the group $G$; then the double character group $D(D G)$ may be regarded as a functor $D \circ D: \mathrm{Abf} \rightarrow \mathrm{Abf}$; the standard construction of the isomorphism $t(G): G \cong D(D G)$ of a group to its double character group is a natural transformation of $I$ to the functor $D \circ D$. On the other hand, there is for each group $G$ an isomorphism $s(G): G \cong D G$ of $G$ to its character group, but this isomorphism depends on a choice of generators in $G$ and can not be natural (for example, is not natural as a transformation $s: I \rightarrow D$, where $D$ is regarded as a functor by restricting the morphisms to isomorphisms). A parallel example is the familiar natural isomorphism of a finite-dimensional vector space to its double dual.

A functor $G: \mathrm{B}^{\mathrm{op}} \rightarrow \mathrm{C}$ is also called a contravariant functor on $\mathrm{B}$ to C. By definition, it is determined by an object function and a mapping function such that always

$$
G\left(1_{B}\right)=1_{G(B)}, \quad G(g f)=G(f) G(g) ;
$$

the essential observation is that the order of the composition is inverted in the second equation. A functor $H: \mathbf{A} \times \mathbf{B} \rightarrow \mathbf{C}$ is also called a (covariant) bifunctor on A, B to C. Such a bifunctor can be determined by functors of one argument in the following fashion (Homology, Proposition I.8.1). Let $F_{B}: \mathbf{A} \rightarrow \mathbf{C}$ and $\mathbf{C}_{A}: \mathbf{B} \rightarrow \mathbf{C}$ be functors, given for each object $B$ in $\mathbf{B}$ and each $A$ in $\mathbf{A}$. If always $F_{B}(A)=G_{A}(B)$, and if, for each pair of morphisms $f: A \rightarrow A^{\prime}$ and $g: B \rightarrow B^{\prime}$, we have the commutativity

$$
F_{B^{\prime}}(f) G_{A}(g)=G_{A^{\prime}}(g) F_{B}(f): F_{B}(A) \rightarrow F_{B^{\prime}}\left(A^{\prime}\right),
$$

then there is a (unique) bifunctor $H$ with $H(A, B)=G_{A}(B)$ and $H(f, g)=F_{B^{\prime}}(f) G_{A}(g)$. Every bifunctor $H: \mathbf{A} \times \mathbf{B} \rightarrow \mathbf{C}$ can be so described. By the same token if $K$ is a second such bifunctor, a transformation $t: H \rightarrow K$ is natural if it is natural in each variable (in $\mathbf{A}$ or B) separately. Multifunctors with mixed variance can be constructed similarly.

In particular, $\operatorname{hom}(A, B)$ for any category $\mathbf{C}$ may be regarded as a bifunctor. Indeed, morphisms $f: A^{\prime} \rightarrow A$ and $g: B \rightarrow B^{\prime}$ yield "induced" maps

$$
f^{*}: \operatorname{hom}(A, B) \rightarrow \operatorname{hom}\left(A^{\prime}, B\right), \quad g_{*}: \operatorname{hom}(A, B) \rightarrow \operatorname{hom}\left(A, B^{\prime}\right)
$$


of sets, defined for $h: A \rightarrow B$ as $f^{*}(h)=h \circ f$ and $g_{*}(h)=g \circ h$. Since $f^{*} g_{*}=g_{*} f^{*}$, as in (5), hom ${ }_{\mathrm{C}}: \mathrm{Cop}^{\mathrm{op}} \times \mathbf{C} \rightarrow$ Ens is a functor to the category of sets, contravariant in the first argument and covariant in the second. By fixing the first or the second argument we get two functors of a single variable,

$$
\begin{array}{rlrl}
h_{A}: \mathrm{C} & \rightarrow \text { Ens, } & h_{A}(B) & =\operatorname{hom}_{\mathrm{C}}(A, B), \\
h^{B}: \mathrm{Cop} & \rightarrow \text { Ens, } & h^{B}(A)=\operatorname{hom}_{\mathrm{C}}(A, B),
\end{array}
$$

called, respectively, the covariant and the contravariant homfunctors for the category $\mathbf{C}$.

As Freyd has observed, "category" has been defined in order to be able to define "functor" and "functor" has been defined in order to be able to define "natural transformation." Now note that the definition of a category is so constructed that each function $f: X \rightarrow A$ (in the category of sets or in some other category) must have, not only a definite set $X$ as domain, but also a definite set $A$ as range or codomain-and this codomain is by no means the same thing as the image of the set $X$ under the function $f$. For example, let $A$ be a subgroup of the abelian group $B$. Take a set of pairs which is the graph of a function; that is, a subset $P \subset X \times A$ which contains to each $x \in X$ exactly one pair $(x, a)$. Now $P$ determines a function $f: X \rightarrow A$ and also a function $f^{\prime}: X \rightarrow B$, but we do not follow the once frequent practice of identifying these two functions. They must count as different functions if we are to have functors. To show this, take a third abelian group $G$ and recall the well-known fact that $A$ a subgroup of $B$ need not imply that the tensor product $A \otimes G$ is a subgroup of $B \otimes G$-nonzero elements of finite order in $A \otimes G$ may become zero in $B \otimes G$. If the tensor product is to be a functor, then group homomorphisms $f: X \rightarrow A$ and $f^{\prime}: X \rightarrow B$ must induce, under this functor, corresponding group homomorphisms $f \otimes 1: X \otimes G \rightarrow A \otimes G$ and $f^{\prime} \otimes 1$ : $X \otimes G \rightarrow B \otimes G$. Were $f$ identified with $f^{\prime}$, this simply wouldn't work because $A \otimes G$ need not be contained in $B \otimes G$. The same point is more vivid in the case of topological functors: $A$ a subspace of $B$ by no means implies that the homology of $A$ is a subgroup of the homology of $B$. More technically, a functor need not carry monics to monics.

5. Operations on functors. If $F_{i}: \mathbf{B}_{i} \rightarrow \mathbf{C}_{i}$, for $i=1,2$, are functors, their product is the functor

$$
F_{1} \times F_{2}: \mathrm{B}_{1} \times \mathrm{B}_{2} \rightarrow \mathrm{C}_{1} \times \mathrm{C}_{2},
$$

defined on ordered pairs of objects and of morphisms by 


$$
\begin{aligned}
\left(F_{1} \times F_{2}\right)\left(B_{1}, B_{2}\right) & =\left(F_{1}\left(B_{1}\right), F_{2}\left(B_{2}\right)\right), \\
\left(F_{1} \times F_{2}\right)\left(f_{1}, f_{2}\right) & =\left(F_{1} f_{1}, F_{2} f_{2}\right) .
\end{aligned}
$$

If $t_{i}: F_{i} \rightarrow G_{i}$ are natural transformations, for $i=1,2$, their product is the natural transformation $t_{1} \times t_{2}: F_{1} \times F_{2} \rightarrow G_{1} \times G_{2}$ defined on pairs of objects by

$$
\left(t_{1} \times t_{2}\right)\left(B_{1}, B_{2}\right)=\left(t_{1}\left(B_{1}\right), t_{2}\left(B_{2}\right)\right) .
$$

If the $t_{i}$ are natural equivalences, so is $t_{1} \times t_{2}$. The constructions $F_{1} \times F_{2}$ and $t_{1} \times t_{2}$ are, respectively, the object function and the mapping function of the bifunctor "product" on the "functor category" $\mathbf{C}^{\mathbf{B}}$.

If $\mathbf{B}$ is a small category and $\mathbf{C}$ any category, the functor category $\mathbf{C}^{\mathbf{B}}$ has as objects all functors $F: \mathbf{B} \rightarrow \mathbf{C}$, as morphisms all natural transformations $t: F \rightarrow F^{\prime}$, with the evident composition. For example, following the notation of Lawvere, let 2 be the category with exactly two objects $a$ and $b$ and three morphisms $1_{a}, 1_{b}$, and $h: a \rightarrow b$. Then each functor $F: 2 \rightarrow \mathrm{C}$ is completely determined by the morphism $F(h)$ in $\mathbf{C}$. Hence $\mathbf{C}^{2}$ is just another description of the category Morph(C), introduced above, whose objects are the morphisms of $\mathbf{C}$. Similarly, diagrams on $\mathbf{C}$ of any given form may be construed as the objects of a suitable functor category (cf. Homology, IX.3). Again, complete semi-simplicial complexes are functors on an appropriate category to the category of sets, and, hence, are better called simplicial sets (cf. also "simplicial groups"; Homology, VIII.5). Grothendieck [41], Freyd [34], Mitchell [80], and others have pointed out the advantages in the systematic use of functor categories. The idea may be emphasized by calling a natural transformation a morphism of functors, but only misguided authors would use the miserable mixture "functor morphism."

Among the formal operations on functors and natural transformations are fore and aft substitution. Bénabou (unpublished) has shown that these may be conveniently formulated by operations in a certain category $\mathbf{N a t}$. The objects of $\mathbf{N a t}$ are categories, but a morphism $t: \mathbf{A} \rightarrow \mathbf{A}^{\prime}$ is to be a triple $t=(t, F, G)$, where $F$ and $G$ are functors, $F, G: \mathbf{A} \rightarrow \mathbf{A}^{\prime}$, while $t: F \rightarrow G$ is a natural transformation. If $t^{\prime}: \mathbf{A}^{\prime} \rightarrow \mathbf{A}^{\prime \prime}$ is a second such morphism, the composite, written $t^{\prime} * t: F^{\prime} \circ F$ $\rightarrow G^{\prime} \circ G$, is that natural transformation with

$$
\left(t^{\prime} * t\right)(A)=G^{\prime}(t A) \circ t^{\prime}(F A)=t^{\prime}(G A) \circ F^{\prime}(t A),
$$

where the second equality holds in virtue of the naturality of $t^{\prime}$. Each functor $F: \mathbf{A} \rightarrow \mathbf{A}^{\prime}$ determines a special sort of morphism of Nat; namely, the identity natural transformation $F \rightarrow F$, which we shall 
again denote by $F$. For these special morphisms, $F^{\prime} * F$ is just $F^{\prime} \circ F$, so the $*$-composition includes ordinary composition of functors. It also includes substitution processes; thus $F^{\prime} * t$ is that natural transformation

$$
F^{\prime} * t: F^{\prime} \circ F \rightarrow F^{\prime} \circ G
$$

between composite functors which is defined for objects $A$ in A by "fore" substitution, as $\left(F^{\prime} * t\right)(A)=F^{\prime}(t A)$. Similarly, $t^{\prime} * F$ is an "aft" substitution. Bénabou now observes that, for fixed $A$ and $A^{\prime}$, the class $\mathbf{N a t}\left(\mathbf{A}, \mathbf{A}^{\prime}\right)$ of morphisms of $\mathbf{N a t}$ is itself a category (with objects the functors $F: \mathbf{A} \rightarrow \mathbf{A}^{\prime}$ ). Fixing $\mathbf{A}^{\prime \prime}$ as well, the composition rule $*$ of Nat is a bifunctor

$$
*: \operatorname{Nat}\left(\mathbf{A}^{\prime}, \mathbf{A}^{\prime \prime}\right) \times \operatorname{Nat}\left(\mathbf{A}, \mathbf{A}^{\prime}\right) \rightarrow \operatorname{Nat}\left(\mathbf{A}, \mathbf{A}^{\prime \prime}\right) .
$$

In the appendix of [39], Godement listed five rules for substitution. These rules are restatements of the fact that Nat is a category with the composition $*$ as a bifunctor.

6. Products. In $\mathbf{C}$, let $\left\{A_{j}\right\}$ be a family of objects indexed by a set $J$. Consider the category $\mathrm{C} /\left\{A_{j}\right\}$ whose objects are indexed families $\left\{f_{j}: B \rightarrow A_{j} \mid j \in J\right\}$ of morphisms of $\mathbf{C}$ with a common domain $B$, while a morphism $\left\{f_{j}\right\} \rightarrow\left\{f_{j}^{\prime}\right\}$ in $\mathbf{C} /\left\{A_{j}\right\}$ is a morphism $h: B \rightarrow B^{\prime}$ of $\mathbf{C}$ for which $f_{j}^{\prime} h$ is $f_{j}$. A terminal object in this category is called a product of the $A_{j}$; thus a product consists of an object $P$ of $\mathbf{C}$ together with morphisms $p_{j}: P \rightarrow A_{j}$, for $j \in J$, such that any family of morphisms $f_{j}: B \rightarrow A_{j}$ can be written as $f_{j}=p_{j} h$ for a unique morphism $h: B \rightarrow P$. In other words, any diagram

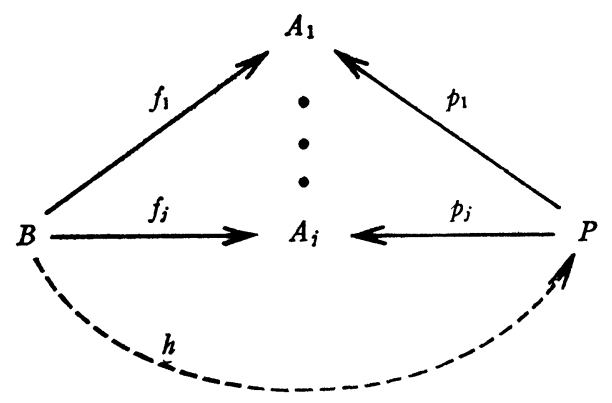

can be filled in by a unique $h$ (at the dotted arrow) so as to be commutative. The product, like any terminal object, is unique up to equivalence (in $\mathrm{C} /\left\{A_{j}\right\}$ ); in particular, the product-object $P$ is unique up to an equivalence in $\mathbf{C}$. It is suggestive to write the product-object as $P=\prod_{j} A_{j}$ and to call the morphisms $p_{j}$ the projections of the product. The product of two objects $A_{1}$ and $A_{2}$ will be 
written $A_{1} \Pi A_{2}$ (it is of ten written $A_{1} \times A_{2}$ ). Note that the very definition of the product asserts that $\left\{f_{j}\right\} \rightarrow h$ is a bijection of sets:

$$
\prod_{j} \operatorname{hom}_{\mathrm{C}}\left(B, A_{j}\right)=\operatorname{hom}_{\mathrm{C}}\left(B, \prod_{j} A_{j}\right) \text {, }
$$

where the symbol $\Pi_{j}$ outside on the left is the cartesian product of sets, while $\Pi$ inside on the right is the categorical product.

For example, in the category Ens of sets, the cartesian product $\prod S_{j}$ of the sets $S_{j}$, together with its projections on each of the factors, is a product in the categorical sense. Similarly, the cartesian product of spaces or the direct product of groups, each with its projections, is a product in the corresponding category. In the category Cat of small categories, the product category with its projections as in (4.2) is a categorical product.

Suppose now that any two objects in the category $\mathbf{C}$ have a product. Then an iterated product $A_{1} \Pi\left(A_{2} \Pi A_{3}\right)$, equipped with the evident projections, is clearly a product. Hence the product is associative and commutative in the evident sense, and any finite family of objects of $\mathbf{C}$ has a product. To each pair of objects $A_{1}, A_{2}$ of $\mathbf{C}$ choose a product $\left\{p_{j}: A_{1} \Pi A_{2} \rightarrow A_{j}\right\}$. Then, to morphisms $f_{j}: A_{j} \rightarrow A_{j}^{\prime}$ of $\mathbf{C}$, there exists a unique morphism $h: A_{1} \Pi A_{2} \rightarrow A_{1}^{\prime} \Pi A_{2}^{\prime}$ with $p_{j}^{\prime} h=f_{j} p_{j}$ for $j=1,2$. The definition $h=f_{1} \Pi f_{2}$ then makes $\Pi$ a functor on $\mathbf{C} \times \mathbf{C}$ to $\mathbf{C}$.

The dual notion is that of a coproduct of the objects $A_{j}$ : A diagram $\left\{k_{j}: A_{j} \rightarrow S\right\}$ which is initial in the category of such diagrams. A coproduct in the category of sets is the disjoint union, in the category of groups the free product, in the category of pointed topological spaces the wedge (the given spaces joined at the base point). If we write the coproduct of objects $\left\{A_{j}\right\}$ as $\amalg A_{j}$, the definition of the coproduct provides a bijection of sets

$$
\prod_{j} \operatorname{hom}_{\mathrm{C}}\left(A_{j}, B\right) \cong \operatorname{hom}_{\mathrm{C}}\left(\mathrm{II} A_{j}, B\right) .
$$

Closely related to the product is the "pull-back" diagram. In a category $\mathrm{C}$ let there be given two morphisms $g_{1}: A \rightarrow C$ and $g_{2}: A_{2} \rightarrow C$ with a common codomain in $C$. Consider all completions of these two morphisms to a commutative square of the form

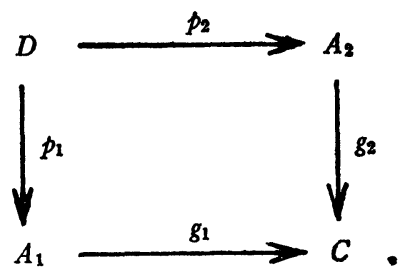


Holding the data fixed, these squares constitute a category with the evident morphisms (morphisms $d: D \rightarrow D^{\prime}$ with $p_{i}^{\prime} d=p_{i}$ for $i=1,2$ ). A terminal object in this category is called a pull-back diagram or a co-universal square. This construction is a familiar one; for example, if $g_{2}: A_{2} \rightarrow C$ is a fibre map of topological spaces or of fibre bundles, then $p_{2}: D \rightarrow A_{2}$ is the fibre map "induced" by the given map $g_{2}$ on the base spaces, or the "induced" bundle, as the case may be. (Indeed, the French translation of "pull-back" is "produit fibré.") In most of the familiar categories (sets, spaces, groups) the pull-back diagram always exists.

The dual of the pull-back diagram is called a push-out diagram or a universal square. More elaborate "universal" problems are possible, but they have apparently not yet been subject to terminological organization, gallic or anglo-saxon.

Given two morphisms $f, g: A \rightarrow B$ in a category $\mathbf{C}$, consider the new category with objects those morphisms $k$ with codomain $A$ and such that $f k=g k$. A terminal object $k$ in this category is called a difference kernel (or an equalizer) of $f$ and $g$. The dual notion is that of a difference cokernel (or co-equalizer). The category $\mathbf{C}$ is called complete ("left complete") if every pair of maps in $\mathbf{C}$ has a difference kernel and every set of objects has a product. If $\mathbf{C}$ and $\mathbf{C}^{\prime}$ are both left complete categories, a functor $F: \mathbf{C} \rightarrow \mathbf{C}^{\prime}$ is called (left) continuous if it preserves all difference kernels and all products.

7. Representable functors. The important notion of a representable functor is due to Grothendieck [42], [43], [44]; for another exposition, see Dold [13].

Let $S: \mathrm{C} \rightarrow$ Ens be a functor (to the category of sets). A representation $(R, \phi)$ of $S$ is a pair, $(R, \phi)$, which consists of an object $R$ of $\mathbf{C}$ and a family $\phi$ of equivalences

$$
\phi(C): \operatorname{hom}_{\mathrm{C}}(R, C) \cong S(C),
$$

natural in $C$. When $S$ has such a representation, it is said to be representable, or to be represented by the object $R$. For each object $R$ of $\mathbf{C}$ we write $h_{R}: \mathbf{C} \rightarrow$ Ens for the covariant homfunctor $h_{R}(C)$ $=\operatorname{hom}_{\mathrm{C}}(R, C)$. A representation $(R, \phi)$ of $S$ is then a natural equivalence of functors

$$
\phi: h_{R} \cong S \text {. }
$$

For example, let $N: \mathrm{Gr} \rightarrow$ Ens be the functor which assigns to each group $G$ its underlying set and to each group homomorphism $f$ the same mapping regarded just as a function on the underlying sets (such a functor $N$ is called a forgetful functor; it "forgets" the group struc- 
ture). This functor $N$ is representable; indeed, it is represented by the additive group $Z$ of integers, for we have the familiar bijection

$$
\operatorname{hom}_{\mathrm{Gr}}(Z, G) \cong S(G),
$$

which assigns to each morphism $f: Z \rightarrow G$ the image $f(1)$ of the generator 1 of $Z$.

For any two objects $A_{1}, A_{2}$ in a category $\mathrm{C}$ define the functor $F: \mathrm{C} \rightarrow$ Ens as

$$
F(C)=\operatorname{hom}_{\mathrm{C}}\left(A_{1}, C\right) \times \operatorname{hom}_{\mathrm{C}}\left(A_{2}, C\right) .
$$

If the category $\mathbf{C}$ has coproducts, (6.2) shows this functor representable by the coproduct $A_{1} \amalg A_{2}$. Again, consider a pair of maps $f_{i}: B \rightarrow A_{i}$, for $i=1,2$, in a category $\mathrm{C}$. The functor

$$
F(C)=\left\{\left(g_{1}, g_{2}\right) \mid g_{i}: A_{i} \rightarrow C \text { and } g_{1} f=g_{2} f\right\}
$$

is represented by the object $A_{1} \amalg_{B} A_{2}$ of the push-out diagram, if the latter exists.

Representable functors may alternatively be described in terms of certain "universal" elements. Given $S: \mathrm{C} \rightarrow$ Ens let $\mathrm{C}_{S^{*}}$ be the category with objects those pairs $(A, x)$ for which $x \in S(A)$, and with morphisms $f:(A, x) \rightarrow(B, y)$ those morphisms $f: A \rightarrow B$ of $\mathbf{C}$ such that $S(f) x=y$. Call this category $\mathrm{C}_{S^{*}}$ the category of $S$-pointed objects of C. Now define a universal point for a functor $S: \mathrm{C} \rightarrow$ Ens to be an object $(R, u)$ initial in the category $\mathbf{C}_{S^{*}}$.

THEOREM 7.1. For each functor $S: \mathrm{C} \rightarrow$ Ens, the formulas

$$
u=(\phi R) 1_{R}, \quad(\phi c) h=(S h) u,
$$

the latter for any morphism $h: R \rightarrow C$, establish a one-one correspondence between representations $(R, \phi)$ of $S$ and universal points $(R, u)$ for $S$.

Proof. First, let $(R, \phi)$ be a representation of $S$. Since $\phi$ is natural, the diagram

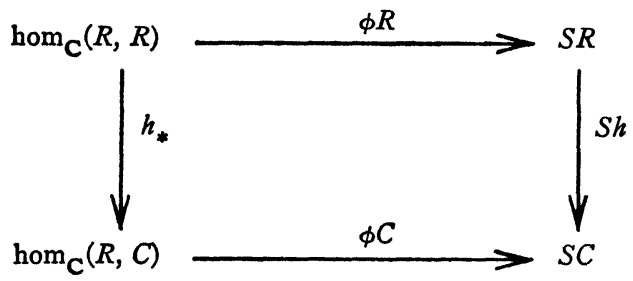

commutes for each $h: R \rightarrow C$. Write $u$ for the image of $1_{R}$ under $\phi R$; the commutation now states that $(\phi C) h=(S h) u$. Since $\phi C$ is assumed 
invertible, this asserts that each element of the set $S C$ can be written uniquely as $(S h) u$ for some $h$; hence $(R, u)$ is a universal point for $S$.

Conversely, let $(R, u)$ be a universal point for $S$. Define $\phi$ by $(\phi C) h=(S h) u$. The universality of $u$ then states that $\phi C$ so defined is a bijection $\operatorname{hom}_{\mathrm{C}}(R, C) \rightarrow S C$ of sets. It is clearly natural, hence is the required representation of the functor $S$.

For example, in the case of the functor represented by the coproduct above, a universal point of $F(C)$ is the pair of injections $i_{j}: A_{j} \rightarrow A_{1} \amalg A_{2}$ of the factors into the coproduct.

As in the case of an initial object in any category, a universal point for $S$ is unique up to an equivalence (of $R$ ). Hence we have

CoROLlaRy 7.2. If $(R, \phi)$ and $\left(R^{\prime}, \phi^{\prime}\right)$ are two representations of the same functor $S$, there is an equivalence $\theta: R \rightarrow R^{\prime}$ of $\mathrm{C}$ such that $\phi \theta^{*}=\phi^{\prime}$.

Corollary 7.3. Let the functors $S, S^{\prime}: \mathrm{C} \rightarrow$ Ens have representations $(R, \phi)$ and $\left(R^{\prime}, \phi^{\prime}\right)$, respectively. Then to each natural transformation $t: S \rightarrow S^{\prime}$ there is a unique morphism $\rho: R^{\prime} \rightarrow R$ of $\mathrm{C}$ such that

$$
\iota \phi=\phi^{\prime} \rho^{*}: \operatorname{hom}_{\mathrm{C}}(R, C) \rightarrow S^{\prime}(C) .
$$

Proof. Let $(R, \phi)$ correspond as in Theorem 7.1 to the universal point $(R, u)$. Then $(R,(t R) u)$ is an $S^{\prime}$-pointed object of $\mathbf{C}$. Since $\left(R^{\prime}, u^{\prime}\right)$ is a universal point, there is a unique morphism $\rho: R^{\prime} \rightarrow R$ such that $\left(S^{\prime} \rho\right) u^{\prime}=(t R) u$. By the correspondence (3), this condition translates to (4).

The conclusion of this corollary may also be formulated as a bijection

$$
\operatorname{Nat}\left(S, S^{\prime}\right) \cong \operatorname{hom}_{\mathrm{C}}\left(R^{\prime}, R\right),
$$

where "Nat" stands for the set of natural transformations from $S$ to $S^{\prime}$.

The argument for Theorem 7.1 rests on the observation that each transformation,

$$
t_{C}: \operatorname{hom}_{\mathrm{C}}(R, C) \rightarrow S(C),
$$

natural in $C$, is completely determined by the image $t_{R}\left(1_{R}\right)$. This is the

Lemma 7.4 (Yoneda Lemma). For each object $C \in \mathrm{C}$ and each functor $S: \mathrm{C} \rightarrow \mathrm{Ens}$, there is a bijection

$$
\psi: S(C) \cong \operatorname{Nat}\left(h_{C}, S\right) .
$$

The map $\psi$ is defined for $x \in S(C)$ and $f \in h_{C}(A)$ as $(\psi x) f=(S f) x$. There is a corresponding result for functors $W: \mathbf{C}^{\mathrm{op}} \times \mathbf{C} \rightarrow$ Ens. Define a diagonal spread for $W$ to be a function $d$ which assigns to each object $M$ an element $d_{M} \in W(M, M)$ such that 


$$
W(1, k) d_{M}=W(k, 1) d_{N} \in W(M, N)
$$

holds for each morphism $k: M \rightarrow N$ of $\mathbf{C}$. Then

Lemma 7.5 (Diagonal Yoneda). For each bifunctor $W: \mathrm{C}^{\circ} \times \mathbf{C}$ $\rightarrow$ Ens, there is a one-one correspondence between natural transformations $t:$ hom $_{\mathrm{C}} \rightarrow W$ of bifunctors and diagonal spreads $d$ for $W$. Given $t$, $d_{M}$ is $t\left(1_{M}\right)$; given $d, t(k)$ is $W(1, k) d_{M}$.

Proof. By the previous lemma, $t$ is determined by $d$. Naturality requires for each $k: M \rightarrow N$ the commutativity of the diagram

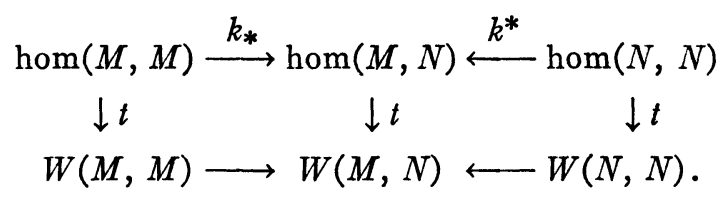

Putting identities at the ends of the top rows gives $1 \rightarrow k \leftarrow 1$; hence the bottom row is $d_{M} \rightarrow t k \leftarrow d_{N}$, as required.

As another example, let $F, G: \mathbf{B} \rightarrow \mathbf{C}$ be functors, while $W: \mathbf{B}^{\circ \mathrm{p}} \times \mathbf{B}$ $\rightarrow$ Ens is the bifunctor given as

$$
W(A, B)=\operatorname{hom}_{\mathrm{C}}(F A, G B) .
$$

A diagonal spread $t$ for $W$ is, then, a function assigning to each object $B$ a morphism $t(B): F B \rightarrow G B$ such that, for every $f: B \rightarrow B^{\prime}, G(f) t(B)$ $=t\left(B^{\prime}\right) F(t)$-in other words, a diagonal spread for this functor is exactly the same thing as a natural transformation $t: F \rightarrow G$.

Consider now bifunctors $T(M, A)$ which are representable when regarded as functors of the second argument $A$.

Theorem 7.6. Let $\mathrm{M}$ and $\mathrm{A}$ be categories and

$$
\mathrm{T}: \mathrm{M}^{\mathrm{op}} \times \mathbf{A} \rightarrow \text { Ens }
$$

a bifunctor such that for each object $M$ the functor $T(M,-)$ has a representation $\left(R_{M}, \phi_{M}\right)$. There is then a unique functor $R: \mathbf{M} \rightarrow \mathbf{A}$ with object function $R(M)=R_{M}$ such that the equivalence

$$
\phi_{M}: \operatorname{hom}_{\mathrm{A}}\left(R_{M}, A\right) \cong T(M, A)
$$

is natural in $\mathbf{M}$ as well as in $\mathbf{A}$.

Alternatively, if each functor $T(M,-)$ has a universal point $\left(R_{M}, u_{m}\right)$, there is a unique functor $R: \mathrm{M} \rightarrow \mathrm{A}$ such that $u$ is a diagonal spread for the bifunctor $T\left(M, R_{N}\right)$.

PROOF. To any morphism $k: M \rightarrow N$ we must choose a morphism $R k: R_{M} \rightarrow R_{N}$ to make $\phi_{M}$ natural; that is, to render commutative the diagram 


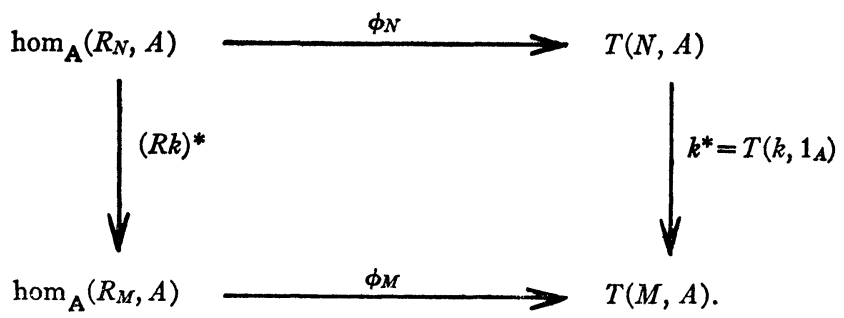

Now put in the definitions of $\phi_{M}$ and $\phi_{N}$ in terms of the universal points $\left(R_{M}, u_{M}\right)$ for $T(M,-)$; this naturality condition then reduces to the special case with $A=R_{N}$ and $1: R_{N} \rightarrow R_{N}$ the identity. In this case the condition reads $(R k)_{*} u_{M}=k^{*} u_{N}$, as in the diagram,

$$
u_{M} \in T\left(M, R_{M}\right) \stackrel{(R k)_{*}}{\longrightarrow} T\left(M, R_{N}\right) \stackrel{k^{*}}{\longleftarrow} T\left(N, R_{N}\right) \ni u_{N},
$$

where $(R k)_{*}$ is short for $T\left(1_{M}, R k\right)$ and $k^{*}$ for $T\left(k, 1_{R_{N}}\right)$. But $\left(R_{M}, u_{M}\right)$ is a universal point for the functor $T(M,-)$, while $\left(R_{N}, k^{*} u_{N}\right)$ is another "point" for this functor; hence there is a unique morphism $g: R_{M} \rightarrow R_{N}$ with $g_{*} u_{M}=k^{*} u_{N}$. Thus the choice $R(k)=g$ is forced; with this choice, it is clear that $R$ becomes a functor $\mathbf{M} \rightarrow \mathbf{A}$ as desired.

The universal points for the functors $T(M,-)$ may also be interpreted as "universal maps" in a sense that was first proposed by Samuel [89]. For categories $\mathbf{M}$ and $\mathbf{A}$ let there be given a function "map" which assigns to each pair of objects $M \in \mathbf{M}$ and $A \in \mathbf{A}$ a set $\operatorname{map}(M, A)$ of "maps" $s$ from $M$ to $A$. Assume also that these "maps" compose in the usual fashion with morphisms of $\mathbf{M}$ or of $\mathbf{A}$, so that $s \in \operatorname{map}(M, A)$ and $f: N \rightarrow M$ or $\alpha: A \rightarrow B$ determine $s f \in \operatorname{map}(N, A)$ and $\alpha s \in \operatorname{map}(M, B)$ with $s 1_{M}=s=1_{A} s$ with all the usual associativity axioms. Given an object $M$, an element $u \in \operatorname{map}(M, R)$ is "universal" for these data if, to each $s \in \operatorname{map}(M, A)$, there is a unique $\alpha: R \rightarrow A$ with $\alpha u=s$, as in the diagram

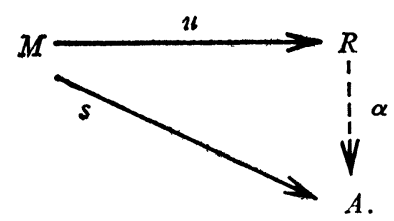

This fits the previous theory. Indeed, the given data on the sets of "maps" are exactly what is required to make $\operatorname{map}(M, A)$ a functor $\mathbf{M}^{\mathrm{op}} \times \mathrm{A} \rightarrow$ Ens, while a "universal map" $u: M \rightarrow R$ is exactly a universal point $(R, u)$ for the functor $\operatorname{map}(M,-)$.

The "universal maps" include many familiar instances, such as the 
construction of tensor products. To describe the latter case, let $\mathbf{A}$ be the category of modules (over some commutative ring), while $\mathbf{M}=\mathbf{A} \times \mathbf{A}$. Take $\operatorname{map}(M, A)=\operatorname{map}(B \times C, A)$ to be the set of all bilinear maps $s: B \times C \rightarrow A$, with the evident composition with morphisms. Then the function $(b, c) \rightarrow b \otimes c$ provides a universal map $\otimes: B \times C \rightarrow B \otimes C$.

In the above discussion, the replacement of $\mathrm{C}$ by its opposite will yield a description of the representation of contravariant functors $K: \mathbf{C o p}_{\rightarrow} \rightarrow$ Ens. In detail, a corepresentation of $K$ is a pair $(R, \psi)$, where $R$ is an object of $\mathrm{C}$ and $\psi(C)$ a family of equivalences

$$
\psi(C): \operatorname{hom}_{\mathrm{C}}(C, R) \cong K(C)
$$

natural in $C$; in other words, $\psi$ is a natural equivalence $\psi: h^{R} \cong K$, where $h^{R}(C)=\operatorname{hom}(C, R)$ is the contravariant hom functor. Now take a $K$-copointed object of $\mathbf{C}$ to be a pair $(A, x)$ with $A$ an object of $\mathbf{C}$ and $x \in K(A)$, while a morphism $f:(A, x) \rightarrow(B, y)$ of such objects is a morphism $f: A \rightarrow B$ of $\mathbf{C}$ with $K(f) y=x$. A co-universal copoint for the functor $K$ is then defined as a terminal object $(R, v)$ in the category of $K$-copointed objects of $\mathrm{C}$. The formulas

$$
v=(\psi R) 1_{R}, \quad \psi g=(K g) v, \text { for } g: C \rightarrow R,
$$

provide a one-one correspondence between corepresentations $(R, \psi)$ of $K$ and co-universal copoints $(R, v)$ for $K$.

8. Adjoints. We now present a basic idea due to Kan. For sets $S, T$, and $U$, the familiar process of writing a function $h: S \times T \rightarrow U$ of two variables as a function $\phi h$ of one variable (in $S$ ) whose values are functions of a second variable (in $T$ ) yields a one-one correspondence

$$
\phi: \operatorname{hom}(S \times T, U) \cong \operatorname{hom}(S, \operatorname{hom}(T, U)) .
$$

This may be regarded as a natural equivalence; indeed, hold $T$ fixed and define functors $F, G$ : Ens $\rightarrow$ Ens by $F(S)=S \times T$ and $G(U)$ $=\operatorname{hom}(T, U)$. Then the correspondence $\phi$ takes the form

$$
\phi_{S, U}: \operatorname{hom}(F(S), U) \cong \operatorname{hom}(S, G(U)) \text {; }
$$

each side is a functor Ens ${ }^{\circ p} \times$ Ens $\rightarrow$ Ens, and $\phi$ is natural (in the arguments $S$ and $U$ ). This formula is reminiscent of the familiar formula $\left(T^{*} f, g\right)=(f, T g)$ describing the adjoint $T^{*}$ of a linear transformation $T$.

In general, consider any two categories $\mathbf{A}$ and $\mathbf{M}$ and functors

$$
F: \mathbf{M} \rightarrow \mathbf{A}, \quad G: \mathbf{A} \rightarrow \mathbf{M} .
$$


Definition. An adjunction $\phi$ of $F$ to $G$ is a natural equivalence

$$
\phi=\phi_{M, A}: \operatorname{hom}_{\mathbf{A}}(F M, A) \cong \operatorname{hom}_{\mathbb{M}}(M, G A) \text {, }
$$

$M \in \mathbf{M}, A \in \mathbf{A}$ ("natural" means that each side is regarded as a functor $\left.\mathbf{M}^{\mathrm{op}} \times \mathbf{A} \rightarrow \mathbf{E n s}\right)$. If there exists such an adjunction call $F$ an adjoint of $G$ or $G$ a coadjoint of $F$. (Earlier terminology had $F$ a "left adjoint" of $G$ and $G$ a "right adjoint" of $F$.)

For each $M$, the adjunction isomorphism (2) states that the functor on the right of (2) is representable; indeed, representable by the pair $\left(F_{M}, \phi_{M}\right)$. Now hom $(M, G A)$, as a functor of $A$, is the composite functor $h_{M} \circ G$, and we know that representable functors may be described in terms of universal points. In the present case, a "point" is a map $f: M \rightarrow G A$, and we shall speak of universal "junctions," to suggest "adjunction."

Definition. A universal junction for a functor $G: \mathbf{A} \rightarrow \mathbf{M}$ is a function $u$ which assigns to each object $M$ an initial $\left(h_{M} \circ G\right)$-pointed object $\left(F_{M}, u_{M}\right)$ of $\mathbf{A}$.

An $\left(h_{M} \circ G\right)$-pointed object of $\mathbf{A}$ is a pair $(A, f)$ where $f: M \rightarrow G A$. The condition that $u_{M}$ be initial may be restated thus: Given any morphism $f: M \rightarrow G A$ of $\mathbf{M}$, the diagram

(3)

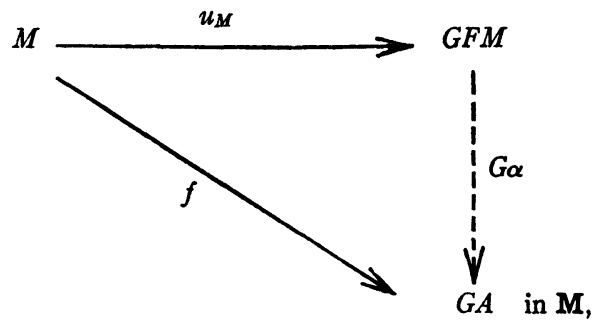

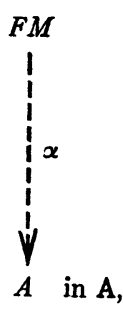

can be filled in uniquely with a morphism $\alpha$ of $\mathbf{A}$ so as to be commutative.

Given a universal junction $u$ for $G$, the alternative form of Theorem 7.6 states that there is a unique functor $F: \mathbf{M} \rightarrow \mathbf{A}$ with object function $F_{M}$ and with $u$ a diagonal spread for $\operatorname{hom}_{\mathbf{M}}(M, G F N)$. This last condition means exactly that $u$ is a natural transformation

$$
u: I \rightarrow G F,
$$

where $I$ is the identity functor of $\mathbf{M}$. If both functors $F$ and $G$ are given in advance, we call $u$ a universal $G-F$ junction (in a previous version of this paper, a "front adjunction" of $F$ to $G$ ). With this terminology, the arguments indicated above give

ThEOREM 8.1 (KAN). Given functors $F$ and $G$ as in (1), the formulas 


$$
u_{M}=\phi\left(1_{F M}\right), \quad \phi(\alpha)=G(\alpha) u_{M},
$$

the latter for any $\alpha: F M \rightarrow A$ in $\mathbf{A}$, establish a one-one correspondence between adjunctions of $F$ to $G$ and universal $G-F$-junctions.

Corollary 8.2. Any two adjoints $F$ and $F^{\prime}$ of $G: \mathbf{A} \rightarrow \mathbf{M}$ are naturally equivalent.

Explicitly, if $u: I \rightarrow G F$ and $u^{\prime}: I \rightarrow G F^{\prime}$ are front adjunctions, there is a natural equivalence $\theta: F \rightarrow F^{\prime}$ such that $u_{M}^{\prime}=G\left(\theta_{M}\right) u_{M}$ for all $M$ in M. Equivalently, if $\phi$ is an adjunction of $F$ to $G$ and $\phi^{\prime}$ one of $F^{\prime}$ to $G$, then there is a natural equivalence $\theta: F \rightarrow F^{\prime}$ such that $\phi^{\prime}$ is the composite

$$
\operatorname{hom}\left(F^{\prime} M, A\right) \stackrel{\left(\theta_{M}\right)^{*}}{\longrightarrow} \operatorname{hom}(F M, A) \stackrel{\phi}{\longrightarrow} \operatorname{hom}(M, G A) .
$$

Theorem 7.6 also allows us to dispense with the assumption that $F$ is a functor:

Proposition 8.3. A functor $G: \mathbf{A} \rightarrow \mathbf{M}$ has an adjoint if and only if, for each object $M$, the functor hom $(M, G A$ ) is representable (as a functor of $A)$. If $\left(F M, \phi_{M}\right)$ is a representation of this functor, then $F$ is the object function of an adjoint, and $\phi_{M}$ is the corresponding adjunction.

Equivalently, $G$ has an adjoint if and only if it has a universal junction $u$.

There is a dual treatment. Let $\phi$ be an adjunction, as in (2). For each object $A,\left(h^{A} \circ F\right)(M)=\operatorname{hom}_{\mathbf{A}}(F M, A)$ is then corepresentable as a functor of $M$. This corepresentation corresponds to a co-universal copoint $\left(G A, u_{A}\right)$ in the category of suitably copointed objects of M. Explicitly, for given $A$, this is the category whose objects are pairs $(M, \beta: F M \rightarrow A)$, for $M$ an object of $\mathbf{M}$ and $\beta$ a morphism of $\mathbf{A}$. Thus we define a co-univeral junction for $F$ to be a function $\mu$ which assigns to each object $A$ a terminal $h^{A} \circ F$-copointed object $\left(G_{A}, \mu_{A}: F G_{A} \rightarrow A\right)$ of $\mathbf{A}$. Then the formulas

$$
\mu_{A}=\phi^{-1}\left(1_{G A}\right), \quad \phi^{-1}(f)=\mu_{A} \circ(F f),
$$

the latter for any $f: M \rightarrow G A$, establish a one-one correspondence between adjunctions $\phi$ of $F$ to $G$ and co-universal junctions $\mu$. Given the functor $F$, a coadjoint, if it exists, is uniquely determined up to natural equivalence, much as in Corollary 8.2. As before,

Proposition 8.4. A functor $F: \mathbf{M} \rightarrow \mathbf{A}$ has a coadjoint if and only if, for each object $A$, the functor $\operatorname{hom}_{\mathrm{A}}(F M, A)$ is corepresentable as a functor of $M$. Also, $F$ has a coadjoint if and only if for each object $A$ 
there is a terminal object in the category of all ( $\left.h^{A} \circ F\right)$-copointed objects of $M$.

The existence of an adjunction may also be described in terms of the transformations $u$ and $\mu$.

Proposition 8.5. The functor $F: \mathbf{M} \rightarrow \mathbf{A}$ is adjoint to $G: \mathbf{A} \rightarrow \mathbf{M}$ if and only if there are natural transformations $u: I_{\mathbf{M}} \rightarrow G F$ and $\mu: F G \rightarrow I_{\mathbf{A}}$ such that

$$
G\left(\mu_{A}\right) u_{G A}=1_{G A} \text { and } \mu_{F M} F\left(u_{M}\right)=1_{F M}
$$

for all objects $A$ of $\mathbf{A}$ and $M$ of $\mathbf{M}$.

Proof. First recall that an adjunction $\phi$ gives $u$ and $\mu$ in the following symmetric fashion. To obtain $u_{M}$, set $A=F M$ in (2) and apply $\phi$ to $1_{F M}$; to obtain $\mu_{A}$, set $M=G A$ and apply $\phi^{-1}$ to $1_{G A}$. Now $\mu_{A}=\phi^{-1}\left(1_{G A}\right)$ becomes $1_{G A}=\phi\left(\mu_{A}\right)$; with the formula (4) for $\phi$, this gives the first equation displayed above; the derivation of the second equation is symmetric.

Conversely, given natural transformations $u$ and $\mu$, we define maps

$$
\operatorname{hom}_{\mathbf{A}}(F M, A) \underset{\psi}{\stackrel{\phi}{\rightleftarrows}} \operatorname{hom}_{\mathbf{M}}(M, G A)
$$

as $\phi(\alpha)=G(\alpha) u_{M}$ for $\alpha: F M \rightarrow A$ and $\psi(f)=\mu_{A} F(f)$ for $f: M \rightarrow G A$. Then

$$
\phi \psi(f)=G\left(\mu_{A}\right) G F(f) u_{M}=G\left(\mu_{A}\right) u_{G A} f=f,
$$

since $G$ is a functor and $u$ is natural. Hence $\phi \psi$ is the identity. Dually, $\psi \phi=1$. Therefore $\phi$ is invertible; it follows readily that it is natural.

Examples of adjoints are myriad. In general topology there are many, such as the Stone- Cech compactification (cf. Kennison [62]).

In some instances, the front adjunction provides the most convenient way of recognition. For example, to discuss the free-group functor in the notation of this section, let A stand for the category of groups and $\mathbf{M}$ for that of sets while $G: \mathbf{A} \rightarrow \mathbf{M}$ is the forgetful functor ( $\$ 7)$. Now let $F$ be the functor which assigns to each set $M$ the free group on the elements of the set $M$ as generators; while $u_{M}: M \rightarrow G F M$ is the function which sends each element of $M$ into the same element (in the underlying set of the generated group). The basic construction of the free group on given generators shows that each homomorphism $\alpha: F M \rightarrow A$ of the free group into any other group is completely determined by the induced map $f$ of the set $M$ of generators into (the set of) $A$, and, conversely, that every such set map $f$ extends 
to a group homomorphism. Now these statements say exactly that diagram (3) can be uniquely filled in at $\alpha$, hence that $F$ is the adjoint of $G$.

This example is typical of many: Given an algebraic system $M$ of some type $\mathbf{M}$, the generation from $M$ of a relatively free system $F(M)$ of some other more complicated type $\mathbf{A}$ is just the construction of an adjoint to the natural forgetful functor from $\mathbf{A}$ to $\mathbf{M}$. For example, let $\mathbf{A}$ be the category of rings (always with identity). If we "forget" the addition, we have a functor $G: \mathbf{A} \rightarrow \mathbf{M}$, where $\mathbf{M}$ is the category of monoids (sets with an associative binary operation with anidentity). The adjoint $F(M)$ assigns to each $M$ its monoid ring $Z(M)$; in particular, if $\Pi$ is a group, the ring $Z(\Pi)$ is the usual group ring over the integers (Homology, IV.1 and X.5).

Let $T$ be the functor which assigns to any group $G$ its factor commutator group $T G$. Then any homomorphism from $G$ to an abelian group $A$ factors uniquely through the evident map $G \rightarrow T G$. This gives a bijection

$$
\mathrm{Ab}(T G, A) \cong \operatorname{Gr}(G, N A),
$$

which asserts that $T$ is adjoint to the forgetful functor $N$ from abelian groups to groups.

In the category Mod of modules $A, B, C, \cdots$ over a commutative ring, it is a familiar fact that a bilinear function $f: A \times B \rightarrow C$ on $A \times B$ can be interpreted as a linear function on $A$ to the linear functions on $B$ to $C$; in other words, there is a natural equivalence

$$
\operatorname{hom}(A \otimes B, C) \cong \operatorname{hom}(A, \operatorname{hom}(B, C)) \text {. }
$$

For $B$ fixed, this asserts that the functor $-\otimes B$ is adjoint to hom $(B,-)$.

Similarly, for topological spaces $X, Y$, and $Z$, with the usual compact-open topology on the space $Z^{Y}$ of continuous functions on $Y$ to $Z$ there is, for $Y$ regular and locally compact, a bijection

$$
\operatorname{hom}(X \times Y, Z) \cong \operatorname{hom}\left(X, Z^{Y}\right) ;
$$

thus, for the category Top, $-\times Y$ is adjoint to $(-)^{Y}$. In the category Top* of pointed spaces, $Z^{Y}$ will denote the function space of all basepoint preserving continuous maps of $Y$ to $Z$, again with the compactopen topology. On the other hand, the smash product of $X$ and $Y$ is

$$
X \wedge Y=(X \times Y) /\left(\left(*_{X} \times Y\right) \cup\left(X \times *_{Y}\right)\right)
$$

(the cartesian product with the wedge of $X$ and $Y$ collapsed to a point). The above exponential law in this case becomes a bijection 


$$
\operatorname{hom}(X \wedge Y, Z) \cong \operatorname{hom}\left(X, Z^{Y}\right) ;
$$

$-\wedge Y: \operatorname{Top}_{*} \rightarrow \mathrm{Top}_{*}$ is adjoint to the functor $(-)^{Y}$. In particular, take $Y$ to be the circle $S^{1}$. Then $X \wedge S^{1}$ is the suspension of $X$, while $Z^{S^{1}}$ is the loop space: In Top*, the suspension functor is adjoint to the loop-space functor.

Swan's notes [103] illustrate the many occurrences of adjoints in the theory of sheaves. For example, the functor which assigns to each presheaf (of abelian groups over a space $X$ ) its associated sheaf is adjoint to the forgetful functor from sheaves to presheaves.

The notions of direct and inverse limits may be described effectively in terms of adjoints, following Kan [58]. Thus consider a directed set $D$; that is, a partly ordered set in which there exists to each pair of elements $d_{1}, d_{2}$ an element $d$ with both $d_{1} \leqq d$ and $d_{2} \leqq d$. Take $\mathbf{D}$ to be the category corresponding to the partly ordered set $D$, in the sense described above in $\$ 2$. A directed system of abelian groups over the index set $D$ is then the same thing as a functor $T: D \rightarrow A b$, for such a $T$ assigns to each index $d \in D$ an abelian group $F(d)$ and to each pair of indices $d_{1} \leqq d_{2}$ a morphism $T\left(d_{1}\right) \rightarrow T\left(d_{2}\right)$ of abelian groups, with the evident composition rule. For each abelian group $A$ there is a constant directed system $N A$, defined by taking $(N A) d=A$ for each index $d$ while each morphism $(N A) d_{1} \rightarrow(N A) d_{2}$ is $1_{A}$. Thus $N$ is a functor $N: \mathrm{Ab} \rightarrow \mathrm{Ab}^{\mathbf{D}}$. Now suppose that this functor has an adjoint $L: \mathrm{Ab}^{\mathrm{D}} \rightarrow \mathrm{Ab}$, as in

$$
\phi: \operatorname{hom}_{\mathrm{Ab}}(L T, A) \cong \operatorname{Nat}(T, N A) .
$$

Thus $L$ assigns to each directed system $T$ of abelian groups a "limit group" $L T$, while the universal junction $u_{T}: T \rightarrow N L T$ is a map of directed systems, that is, maps each $T(d)$ into $L(t)$ so that each diagram

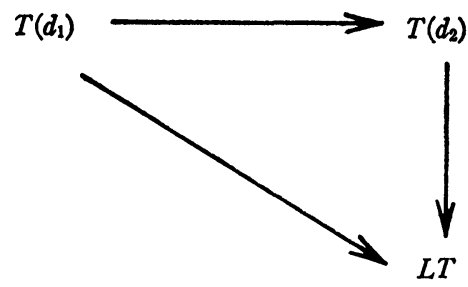

commutes. Moreover, $u$ is universal. This is exactly the description of the direct limit $L T$ of the directed system. Inverse limits (of groups, spaces, etc.) may be handled in dual fashion. Both these types of limits apply to functors $T: D \rightarrow A b$, where $\mathrm{D}$ is the category belong- 
ing to some directed set. The same definitions apply for a functor $T: \mathbf{E} \rightarrow \mathbf{A b}$ on any small category $\mathbf{E}$; the notions corresponding to inverse and direct limits in the more general case are called left and right roots by Freyd [34]. If $\mathrm{E}$ is small and $\mathrm{C}$ left complete, any $T: \mathbf{E} \rightarrow \mathbf{C}$ has a left root (=an inverse limit).

Freyd [34] and also Lawvere [66] give a standard theorem on the existence of an adjoint. Suppose that the categories $\mathbf{A}$ and $\mathbf{M}$ are both left and right complete, in the sense described in $\$ 6$. Then a functor $G: \mathbf{A} \rightarrow \mathbf{M}$ has an adjoint if and only if $G$ commutes with all equalizers and all products and, moreover, there exists to every object $M$ a set $U(M)$ of $\left(h_{M} \circ G\right)$-pointed objects $(A, f)$ of $\mathbf{A}$ with the following property: Given any $\left(h_{M} \circ G\right)$-pointed object $(B, g)$ of $\mathbf{A}$, there exists an object $(A, f)$ in the set $U(M)$ together with a morphism $(A f) \rightarrow(B, g)$ of pointed objects. This last "set-theoretic" condition is clearly necessary if there is to be a front adjunction for $G$. This "adjoint functor theorem" applies to many different categoriesand in many of these categories has been rediscovered in more special form.

"Universality" is frequently useful even without formal employment of adjoints. Thus cohomology operations are defined as natural transformations, and have universal models $K(\Pi, n)$. Also Higgins [46] has shown that systematic use of universality gives a proof of the Kuroš subgroup theorem without the usual complicated cancellation arguments.

\section{Chapter II. Additive Structures on Categories}

9. Kernels and cokernels. A null object in the category $\mathbf{C}$ is an object which is both initial and terminal in $\mathbf{C}$; in other words, $N$ is a null object if each set $\operatorname{hom}(N, C)$ and each $\operatorname{hom}(C, N)$ contain exactly one morphism. Any two null objects of $\mathbf{C}$ are therefore equivalent. If the category $\mathbf{C}$ contains a null object, then for each pair of objects $A$ and $B$ there is exactly one morphism $0_{B}^{A}: A \rightarrow N \rightarrow B$; it is called the zero morphism from $A$ to $B$ and may be described as the unique morphism with domain $A$ and codomain $B$ which "factors through" the null object $N$. Moreover, this morphism $0=0_{B}^{A}$ is independent of the choice of a null object of $\mathbf{C}$. Furthermore, any composite with one factor a zero morphism is itself zero.

If $\mathbf{C}$ has a null object, the notion of "kernel" for any morphism $f: A \rightarrow B$ can be defined: A kernel is any morphism $k: K \rightarrow A$ with codomain $A$ such that:

(i) The composite $f k=0$ (i.e., $=0{ }_{B}^{K}$ );

(ii) If $f h=0$, then $h=k g$ for a unique $g$. 
This second property states that any commutative diagram of the form

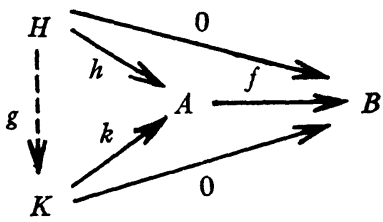

can be filled in uniquely at the dotted arrow $g$; in other words, each right annihilator of $f$ factors uniquely through the kernel $k$. Hence a kernel of $f$, if it exists, is necessively monic. A kernel of $f$ can also be described as a terminal object in a suitable category; namely, in the category $\mathbf{N}_{f}$ whose objects are the morphisms $h: H \rightarrow A$ with codomain $A$ such that $f h=0$ and whose morphisms $g: h \rightarrow h^{\prime}$ are those morphisms $g: H \rightarrow H^{\prime}$ of $\mathbf{C}$ such that $h^{\prime} g=k$. As always for terminal objects, any two kernels of $f$ are equivalent; we write $k=\operatorname{ker} f$ for any one of the kernels of $f$. The equivalence class of the kernel $k$ may be regarded as a subobject ( $\$ 3$ ) of the domain $A$ of $f$. The equivalence class of the domain $K$ of the kernel $k$ is also unique, and might be called the "object-kernel," as this corresponds to the ordinary usage of the word "kernel," say in the category Gr.

A kernel of $f$ may also be described as a difference kernel of $f: A \rightarrow B$ and $0: A \rightarrow B$.

Dually, construct from each $f: A \rightarrow B$ the category ${ }_{f} \mathbf{N}$ whose objects $u: B \rightarrow L$ are the morphisms of $\mathbf{C}$ with domain $B$ and with $u f=0$ and whose morphisms $g: u \rightarrow u^{\prime}$ are the morphisms $g: L \rightarrow L^{\prime}$ of $\mathrm{C}$ with $g u=u^{\prime}$. A cokernel of $f$ is an initial object in this category ${ }_{f} \mathbf{N}$; that is, a cokernel $t$ of $f$ is a morphism $t: B \rightarrow M$ with $t f=0$ such that every left annihilator of $f$ factors uniquely through $t$ :

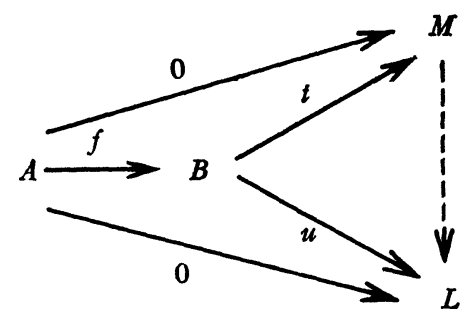

If it exists, the cokernel is epic in $\mathbf{C}$ and is unique up to equivalence (in, $\mathbf{N}$ ).

For example, in the category of groups, a cokernel of $f: G \rightarrow G^{\prime}$ is the canonical projection $p: G^{\prime} \rightarrow G^{\prime} / N$ of $G^{\prime}$ onto the quotient group by the normal subgroup $N$ of $G^{\prime}$ generated by the image $f(G)$. In 
the category Ens* of pointed sets, each morphism $f$ has a kernel and a cokernel:

$$
K \stackrel{k}{\rightarrow} P \stackrel{f}{\rightarrow} Q \stackrel{t}{\rightarrow} M .
$$

For the kernel, take $K=f^{-1}\left(*_{Q}\right)$ and $k$ the injection; for the cokernel, form $M$ from $Q$ by identifying with the base point all points of the image $f(P)$, and take $t$ to be the projection. If $f$ is monic in Ens*, it is a kernel, and $f=\operatorname{ker}(\operatorname{coker} f)$. The dual statement is false because an epic need not be a cokernel, but if $t$ is the cokernel of any map, then $t=\operatorname{coker}(\operatorname{ker} t)$.

10. Additive categories. An additive category is a category $\mathbf{A}$ in which each set hom $(A, B)$ of morphisms has the structure of an abelian group, subject to the following three axioms:

(Add-1) Composition is distributive; that is,

$$
\left(g_{1}+g_{2}\right) f=g_{1} f+g_{2} f, \quad h\left(g_{1}+g_{2}\right)=h g_{1}+h g_{2}
$$

for any $g_{1}, g_{2}: B \rightarrow C, f: A \rightarrow B$, and $h: C \rightarrow D$;

(Add-2) There is a null object $N$;

(Add-3) (Biproducts) To each pair of objects $A_{1}$ and $A_{2}$ there exists an object $B$ and four morphisms

$$
A_{1} \underset{i_{1}}{\stackrel{p_{1}}{\rightleftarrows}} B \underset{i_{2}}{\stackrel{p_{2}}{\rightleftarrows}} A_{2}
$$

which satisfy the identities

$$
p_{1} i_{1}=1_{A_{1}}, \quad p_{2} i_{2}=1_{A_{2}}, \quad i_{1} p_{1}+i_{2} p_{2}=1_{B} .
$$

First some comments. Since each set $\operatorname{hom}_{\mathbf{A}}(A, B)$ of morphisms is an additive group, there is at least one morphism (the zero of this group) from any $A$ to any $B$. By the distributive law, these zero morphisms satisfy $0 f=0$ and $h 0=0$. Hence a null object $N$ of $\mathbf{A}$ may be described as any object $N$ of $\mathbf{A}$ such that $\operatorname{hom}_{\mathbf{A}}(N, N)$ is the zero group, or as any object $N$ such that $1_{N}$ is zero. We often write 0 for a null object. Moreover, a zero morphism $0_{B}^{A}: A \rightarrow B$ in the sense described in $\$ 9$ above is identical with the zero element of the group $\operatorname{hom}_{\mathbf{A}}(A, B)$.

The distributive law shows that a morphism $k$ in an additive category is monic if and only if $k f=0$ always implies $f=0$. Similarly, $t$ is epic if and only if $g t=0$ always implies $g=0$.

The diagram (2) has the following properties (Homology, IX.1). First $p_{1} i_{2}=0$ and $p_{2} i_{1}=0$. If $f_{j}: C \rightarrow A_{j}, j=1,2$, is any pair of morphisms with a common domain $C$, the correspondence $\left(f_{1}, f_{2}\right) \rightarrow g$, where $g=i_{1} f_{1}+i_{2} f_{2}: C \rightarrow B$, provides an isomorphism

$$
\operatorname{hom}\left(C, A_{1}\right) \oplus \operatorname{hom}\left(C, A_{2}\right) \cong \operatorname{hom}(C, B) ;
$$


here $\oplus$ designates the direct sum of abelian groups. Since $p_{j} g=f_{j}$ for $j=1,2$, the diagram $A_{1} \leftarrow B \rightarrow A_{2}$ is a product of $A_{1}$ and $A_{2}$. Similarly, for $h_{j}: A_{j} \rightarrow D$, the correspondence $\left(h_{1}, h_{2}\right) \rightarrow m$, where $m=h_{1} p_{1}+h_{2} p_{2}$, defines an isomorphism

$$
\operatorname{hom}\left(A_{1}, D\right) \oplus \operatorname{hom}\left(A_{2}, D\right) \cong \operatorname{hom}(B, D)
$$

of abelian groups with $m i_{j}=f_{j}$, so $A_{1} \rightarrow B \leftarrow A_{2}$ is a coproduct. Hence we call the diagram (2) a biproduct of $A_{1}$ and $A_{2}$, with projections $p_{j}$ and injections $i_{j}$; given $A_{1}$ and $A_{2}$, it is unique up to an equivalence of $B$ (that is, up to an equivalence in the category of diagrams of the form (2) with fixed ends $A_{1}$ and $A_{2}$ ). We write $B$ as $A_{1} \oplus A_{2}$. Iteration yields biproducts $A_{1} \oplus \cdots \oplus A_{n}=\sum A_{i}$ of any finite number of factors. In particular, an additive category has finite products and finite coproducts. Iteration of the isomorphisms above yields a natural isomorphism

$$
\operatorname{hom}\left(\sum_{1}^{n} A_{i}, \sum_{1}^{m} C_{j}\right) \cong \sum_{i, j=1}^{n, m} \operatorname{hom}\left(A_{i}, C_{j}\right) .
$$

This states that each $f: \sum A_{i} \rightarrow \sum C_{j}$ is determined by the $n \times m$ matrix of its components $f_{i j}: A_{i} \rightarrow C_{j}$; composition is then given by the usual matrix product. Put differently, the system of equations (1) allows a clean and efficient derivation of the usual correspondence between linear transformations and matrices.

If $\mathbf{A}$ and $\mathbf{B}$ are additive categories, a functor $F: \mathbf{A} \rightarrow \mathbf{B}$ is called additive if $F\left(f_{1}+f_{2}\right)=F\left(f_{1}\right)+F\left(f_{2}\right)$ whenever $f_{1}+f_{2}$ is defined. It follows that $F(0)=0, F(-f)=-F(f)$, and $F\left(A_{1} \oplus A_{2}\right) \cong F\left(A_{1}\right) \oplus F\left(A_{2}\right)$. For additive categories all functors considered should normally be additive, since a map $F: \mathbf{A} \rightarrow \mathbf{B}$ should preserve all the structure involved; in particular, the addition. A composite of additive functors is clearly additive. A functor $F$ is additive if and only if it carries every biproduct diagram (2) with properties (3) in A in to a biproduct diagram in $\mathbf{B}$.

A typical example of an additive category is the category $\mathbf{A b}$ of all abelian groups, with the usual homomorphisms, and with the sum of two homomorphisms $f_{1}, f_{2}: A \rightarrow B$ defined as $\left(f_{1}+f_{2}\right) a=f_{1} a+f_{2} a$ for all elements $a$ of the abelian group $A$. Similarly, for each ring $R$ the category $R$-Mod of all left $R$-modules is additive, when the sum of two $R$-module homomorphisms is defined as for abelian groups. In particular, the category of vector spaces over a given field is additive. All these categories have additional properties which make them abelian categories in the sense next to be described. Homological algebra is usually carried out for $R$-modules, though the ideas work 
equally well for bimodules, for sheaves over a topological space, or, more generally, for the objects of any abelian category.

11. Abelian categories. An abelian category $\mathbf{A}$ is an additive category which satisfies the following three additional axioms:

(Abel-1) Every morphism of $\mathbf{A}$ has a kernel and a cokernel;

(Abel-2) For $k$ monic and $t$ epic, $k$ is a kernel of $t$ if and only if $t$ is a cokernel of $k$;

(Abel-3) Every morphism $f$ of $\mathbf{A}$ can be written as a composite $f=m \circ s$ with $m$ monic and $s$ epic.

A short exact sequence

$$
0 \stackrel{0}{\rightarrow} A \stackrel{k}{\rightarrow} B \stackrel{t}{\rightarrow} C \stackrel{0}{\rightarrow} 0
$$

is, by definition, a pair $(k, t)$ of morphisms with codomain $k=$ domain $t$ such that $k$ is a kernel of $t$ and $t$ a cokernel of $k$; in particular, $k$ is monic and $t$ is epic. By (Abel-2), it suffices to require that $k=\operatorname{ker}(t)$ and $t$ is epic, or that $t=\operatorname{coker}(k)$ and $k$ monic. Given any morphism $f: A \rightarrow B$, take the standard factorization $f=m s$ as in (Abel-3) and set $k=\operatorname{ker} f=\operatorname{ker} s, t=\operatorname{coker} f=\operatorname{coker} m$. There results an "analysis" of $f$ in the form of a commutative diagram

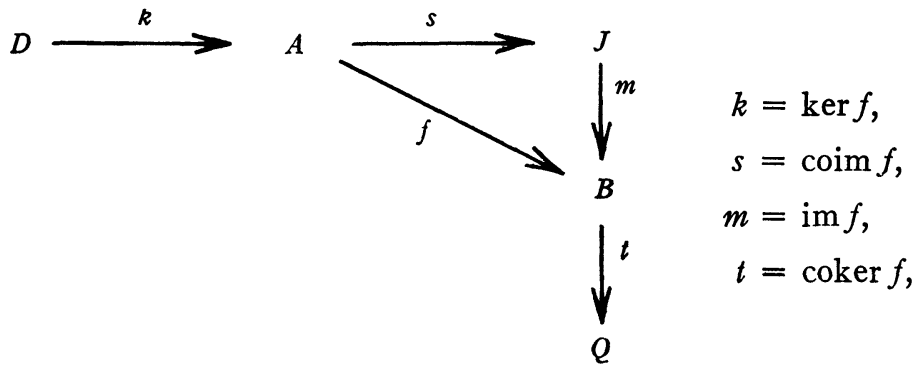

with row and column short exact sequences. This analysis is unique up to equivalence in the category of such diagrams on $f$, that is, up to equivalence in $\mathbf{A}$ of the three objects $D, J$, and $Q$. In the familiar cases, the middle object $J \subset B$ is the image $f(A)$ of $f$; hence we call the monic $m$ the image of $f$; it is unique up to an equivalence of its domain. Alternatively, $J$ may be regarded as a quotient of the domain $A$, so we call $s$ the coimage of $f$-unique up to an equivalence of its codomain.

The usual definition of exactness works without elements. Indeed, a pair of morphisms $A \rightarrow B \rightarrow \rightarrow^{\circ} C$ with domain $g=\operatorname{codomain} f$ is said to be exact at $B$ if and only if it satisfies one (and hence all) of the following equivalent conditions: 
(i) $\operatorname{im} f=\operatorname{ker} g$;

(ii) $\operatorname{coim} g=\operatorname{coker} f$;

(iii) $g f=0$; moreover, whenever $g k=0$ and $h f=0$, then $h k=0$.

For example, since $\operatorname{im} f=\operatorname{ker}(\operatorname{coker} f)$, the condition (i) amounts to requiring that $g$ and coker $f$ have the same right annihilators; in (iii), $g f=0$ implies that every right annihilator of coker $f$ is a right annihilator of $g$, while the second condition of (iii) gives the reverse implication.

A longer sequence of morphisms is exact if it is exact at each intermediate object; in particular, the sequence (1) is short exact if and only if it is exact at $A$, at $B$, and at $C$. Any long exact sequence may be factored into short exact sequences, such as the diagonal sequences in the following commutative diagram.

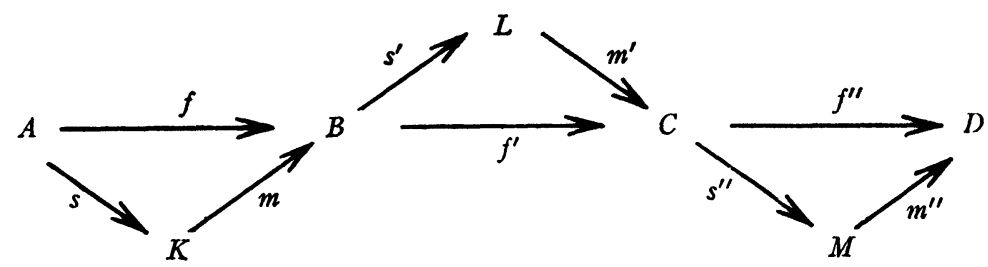

This factorization is the basis of Yoneda's representation of the functor $\operatorname{Ext}^{n}(D, A)$ by congruence classes of long exact sequences from $A$ to $D$ (Homology, III.5 and XII.5).

Given only a diagram like (10.2) which is simultaneously a product and a coproduct in a category $\mathbf{A}$, one can introduce diagonal and codiagonal morphisms $A \rightarrow A \times A \rightarrow A$ and hence (cf. [75]) define an addition of coterminal morphisms of $\mathbf{A}$. Using related devices, Freyd [34] shows that a category $\mathbf{A}$ is abelian if and only if it satisfies the following four axioms: (Add-2), (Abel-1), and

(iii) Every pair of objects has a product and a coproduct;

(iv) Every monic is a kernel and every epic a cokernel.

We often write $k: A \nrightarrow B$ for a monic and $i: B \longrightarrow C$ for an epic. Each biproduct diagram (10.2) yields a short exact sequence $A_{1} \longrightarrow{ }^{i_{1}} B \longrightarrow{ }^{p_{2}} A_{2}$ with a right inverse $i_{2}$ for $p_{2}$ and a left inverse $p_{1}$ for $i_{1}$. Any short exact sequence equivalent to such a biproduct sequence is said to be split. One shows readily that a short exact sequence $A \leadsto{ }^{k} B \longrightarrow{ }^{t} C$ is split if and only if $t$ has a right inverse, or, if and only if $k$ has a left inverse. Moreover, in an abelian category, each pair of morphisms with common codomain has a pull-back.

The product of two additive (or abelian) categories is additive (or abelian), and the opposite of an additive (or abelian) category is 
additive (or abelian, as the case may be). If $\mathbf{B}$ is any small category and $\mathbf{A}$ an abelian category, Grothendieck observed that the functor category $\mathbf{A}^{\mathbf{B}}$, as defined in $\$ 5$, is also an abelian category. Indeed, kernels, cokernels, images, and the like can be defined "termwise" in $\mathbf{A}^{\mathbf{B}}$; for instance, a sequence $F \rightarrow G \rightarrow H$ of natural transformations of functors in $\mathbf{A}^{\mathbf{B}}$ is exact there if and only if every sequence $F(B) \rightarrow G(B)$ $\rightarrow H(B)$ is exact in A. Thus, for example, the category with objects the morphisms of $\mathbf{A}$ is an abelian category, as is the category of long sequences of morphisms of A. However, the category of short exact sequences of an abelian category is not abelian.

12. Additive functors. Consider an additive functor $F: A \rightarrow B$ between abelian categories. We define

$F$ is cokernel preserving (or, right exact) if $\operatorname{coker}(F f)=F(\operatorname{coker} f$ ) for every $f$;

$F$ is kernel preserving (or, left exact) if $\operatorname{ker}(F f)=F(\operatorname{ker} f)$ for every $f$;

$F$ is exact if it is both left and right exact.

A sequence $L \rightarrow \rightarrow^{t} B \rightarrow^{t} C \rightarrow 0$ is called a (short) right exact sequence if it is exact at both $B$ and $C$; that is, if $t=\operatorname{coker} f$. Similarly, a left exact sequence $0 \rightarrow A \rightarrow B \rightarrow D$ is a sequence exact at $A$ and $B$. Consequently, a functor $F$ is right exact if and only if it carries right exact sequences in $\mathbf{A}$ into right exact sequences in $\mathbf{B}$. Dually, $F$ is left exact if and only if it preserves left exact sequences. For that matter, $F$ is left exact if and only if it carries short exact sequences in to left exact sequences, and dually (Homology, Lemma XII.7.1). A functor $F$ is exact if and only if it carries short exact sequences into short exact sequences. An exact functor also satisfies

$$
F(\operatorname{im} f)=\operatorname{im}(F f), \quad F(\operatorname{coim} f)=\operatorname{coim}(F f) .
$$

In any abelian category $\mathbf{A}$, the groups $\operatorname{hom}_{\mathbf{A}}(A, B)$ provide examples of left exact functors to the category $\mathrm{Ab}$ of abelian groups. Indeed, for any $f: A \rightarrow A^{\prime}$, the induced maps

$$
f^{*}: \operatorname{hom}\left(A^{\prime}, B\right) \rightarrow \operatorname{hom}(A, B), \quad f_{*}: \operatorname{hom}(C, A) \rightarrow \operatorname{hom}\left(C, A^{\prime}\right),
$$

defined by composition as in (3.6), are homomorphisms of abelian groups. Hence $\operatorname{hom}(A, B)$ is additive in each argument.

Proposition 12.1. A sequence $0 \rightarrow A \rightarrow^{k} B \rightarrow f D$ is left exact in $\mathbf{A}$ if and only if the sequence

$$
0 \rightarrow \operatorname{hom}(E, A) \stackrel{k_{*}}{\rightarrow} \operatorname{hom}(E, B) \stackrel{f_{*}}{\rightarrow} \operatorname{hom}(E, D)
$$

is a left exact sequence of abelian groups for every object $E$ of A. Simi- 
larly, a sequence $L \rightarrow^{f} B \rightarrow^{t} C \rightarrow 0$ is right exact in $\mathbf{A}$ if and only if every sequence

$$
0 \rightarrow \operatorname{hom}(C, E) \stackrel{t^{*}}{\rightarrow} \operatorname{hom}(B, E) \stackrel{f^{*}}{\rightarrow} \operatorname{hom}(L, E)
$$

is a left exact sequence of abelian groups.

In other words, $\operatorname{hom}\left(A, A^{\prime}\right)$, as a functor of $A^{\prime}$, preserves kernels, while as a (contravariant) functor of $A$ it takes cokernels to kernels. It is customary to summarize these properties in the statement that hom is a left exact functor in each argument separately.

Proof. The first given sequence is left exact if and only if each $g: E \rightarrow B$ with $f g=0$ factors uniquely as $g=k g^{\prime}$; that is, if and only if $f_{*} g=0$ implies $g=k_{*} g^{\prime}$ for a unique $g^{\prime}$; that is, if and only if (1) is left exact. The proof of (2) is similar.

Note that a morphism $f: A \rightarrow A^{\prime}$ is monic in $\mathbf{A}$ if and only if the induced $f_{*}: \operatorname{hom}(C, A) \rightarrow \operatorname{hom}\left(C, A^{\prime}\right)$ is a monomorphism in $\mathrm{Ab}$ for every object $C$, while $f$ is epic in $\mathbf{A}$ if and only if every induced map $f^{*}$ is a monomorphism.

Each functor $F: \mathbf{A} \rightarrow \mathbf{B}$ determines for each pair of objects $A, A^{\prime}$ of A a map of sets

$$
\mathrm{F}_{A, A^{\prime}}: \operatorname{hom}_{\mathbf{A}}\left(A, A^{\prime}\right) \rightarrow \operatorname{hom}_{\mathbf{B}}\left(F A, F A^{\prime}\right),
$$

namely, the map which assigns to each $f: A \rightarrow A^{\prime}$ the corresponding $F(f)$. The functor $F$ is called full if every such map $F_{A, A^{\prime}}$ is a surjection, and faithful (or an embedding functor) if every such map $F_{A, A^{\prime}}$ is an injection. Thus, for $F, \mathbf{A}$, and $\mathbf{B}$ additive, the functor $F$ is faithful if and only if $F(f)=0$ implies $f=0$ for every $f$. For abelian categories, our third description of an exact pair of morphisms shows that a faithful functor carries nonexact sequences to nonexact sequences.

The Lubkin-Heron-Freyd representation theorem asserts that for each small abelian category $\mathbf{A}$ there is an exact and faithful functor $F: \mathrm{A} \rightarrow \mathrm{Ab}$ to the category of abelian groups. A faithful functor carries nonexact sequences to nonexact sequences, so the image under $F$ of a sequence in $\mathbf{A}$ will be exact in $\mathbf{A b}$ if and only if the original is exact in A. Hence this theorem may be used to transfer diagram lemmas, such as the Five Lemma or the $3 \times 3$ Lemma, from the category of abelian groups (where they are easily established by chasing elements) to an arbitrary small abelian category. To include also those diagram lemmas which assert the existence of maps (notably the construction of the connecting homomorphism, say, via the Ker-Coker sequence) one needs the more powerful Freyd-Mitchell full embedding theorem (see [34], [80]). This theorem constructs to each small abelian category $\mathbf{A}$ a ring $R$ and an exact, faithful, and full functor $F: \mathbf{A} \rightarrow R$-Mod 
to the category of $R$-modules. Since $F$ is full, any homomorphism constructed between $R$-modules $F(A)$ and $F\left(A^{\prime}\right)$ (say, a connecting homomorphism) can be carried back to a morphism $A \rightarrow A^{\prime}$ which will be unique, because $F$ is faithful.

13. Additive adjoints. We now reconsider adjoint functors for the case of additive categories $\mathbf{A}$ and $\mathbf{M}$.

Theorem 13.1. If $G: \mathbf{A} \rightarrow \mathbf{M}$ is an additive functor between additive categories, while $\phi$ is an adjunction of a functor $F: \mathbf{M} \rightarrow \mathbf{A}$ to $G$, then, for each pair of objects $M$ and $A$,

$$
\phi: \operatorname{hom}_{\mathbf{A}}(F M, A) \cong \operatorname{hom}_{\mathbf{M}}(M, G A)
$$

is an isomorphism of abelian groups. Moreover, $F$ is additive and right exact, while $G$ is left exact.

Proof. By Theorem 8.1 we may write $\phi(\alpha)$ as $G(\alpha) u_{M}$. For $\alpha, \beta$ : $F M \rightarrow A$, the additivity of $G$ gives

$$
\begin{aligned}
\phi(\alpha+\beta) & =G(\alpha+\beta) u_{M}=[G(\alpha)+G(\beta)] u_{M}=G(\alpha) u_{M}+G(\beta) u_{M} \\
& =\phi(\alpha)+\phi(\beta) .
\end{aligned}
$$

Therefore $\phi$ is a homomorphism of abelian groups. Next take $f, g: M \rightarrow N$ in $\mathbf{M}$. Since $u: I \rightarrow G F$ is natural,

$$
[G F(f+g)] u_{M}=u_{N}(f+g)=u_{N} f+u_{N} g .
$$

On the other hand, since $G$ is additive,

$$
\begin{aligned}
G[F(f)+F(g)] u_{M} & =[G F(f)+G F(g)] u_{M}=G F(f) u_{M}+G F(g) u_{M} \\
& =u_{N} f+u_{N} g .
\end{aligned}
$$

The identity of these two results and the universal property of $u_{M}$ show that $F(f+g)=F(f)+F(g)$. Hence $F$ is additive.

Now consider a right exact sequence $L \rightarrow M \rightarrow^{t} N \rightarrow 0$ in $\mathbf{M}$ and the corresponding sequence

$$
F(L) \stackrel{F(f)}{\longrightarrow} F(M) \stackrel{F(t)}{\longrightarrow} F(N) \longrightarrow 0
$$

in A. Apply the functor hom to get the commutative diagram

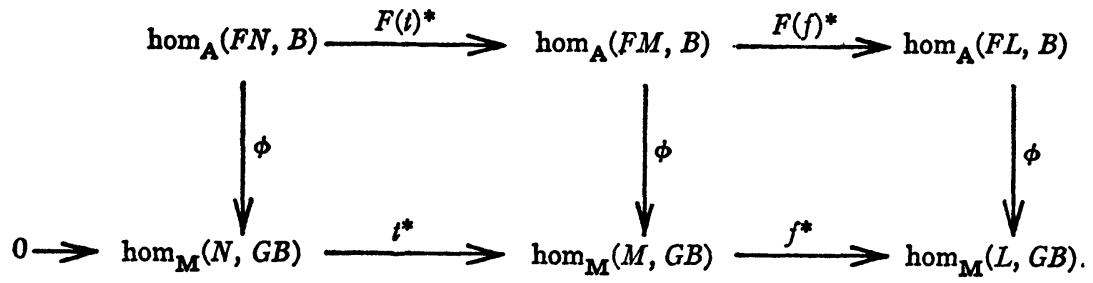


By assumption and the left exactness of hom, the bottom row is left exact. Since each $\phi$ is an isomorphism, the two rows are isomorphic, hence the top row is also left exact. Therefore, by (2) of Proposition 12.1 , the sequence (1) is right exact, and so is $F$. The proof that $G$ is left exact is similar.

14. Differential graded objects. Abelian categories are useful because, for many purposes, "module" may be replaced by "object of an abelian category." This point will now be illustrated in the case of the standard construction of homology groups. Now homology groups are typically calculated from "chain complexes"; a chain complex $X$ has for each degree $n$ a module $X_{n}$ of "chains" of degree $n$ and a boundary homomorphism $d: X_{n} \rightarrow X_{n-1}$ with $d^{2}=0$. Thus a chain complex is graded (by degrees $n$ ) and has a differential $d$-in brief, is a DG-module. Similarly, we have DG-whatnots, with "whatnots" meaning "objects of an abelian category."

Let $\mathbf{A}$ be any abelian category. A graded object for $\mathbf{A}$ is by definition an indexed sequence $X_{0}, X_{1}, X_{2}, \cdots$ of objects of $\mathbf{A}$. Such a sequence may also be described as a functor $X: \mathbf{N} \rightarrow \mathbf{A}$, where $\mathbf{N}$ is the category with objects all natural numbers $0,1,2, \cdots$ and with no morphisms (except the identities). This description of a graded object as a functor automatically tells us that a map $f: X \rightarrow Y$ of graded objects is a natural transformation of functors; that is, an indexed family $\left\{f_{n}: X_{n} \rightarrow Y_{n} \mid n=0,1, \cdots\right\}$ of morphisms of $\mathbf{A}$. Moreover, it also tells us that the class $\mathrm{G}(\mathbf{A})$ of all graded objects for $\mathbf{A}$ is an abelian category.

If $X$ and $Y$ are graded objects, a morphism $f: X \rightarrow Y$ of degree $k$ is a family $\left\{f_{n}: X_{n} \rightarrow Y_{n+k} \mid n=0,1, \cdots\right\}$ of morphisms of $\mathbf{A}$ (set $Y_{m}=0$ if $\left.m<0\right)$. The graded objects with these morphisms again form a category, larger than $\mathrm{G}(\mathbf{A})$. It is not quite an abelian category (coterminal morphisms of different degrees cannot be added, and there is an ambiguity about the degree of the kernel of a morphism); however, it makes sense to talk of exact sequences in this category.

Again, let $\mathbf{A}$ be any abelian category. A DG-object for $\mathbf{A}$ is a diagram

$$
X_{0} \stackrel{d_{1}}{\leftarrow} X_{1} \stackrel{d_{2}}{\leftarrow} X_{2} \stackrel{d_{3}}{\leftarrow} X_{3} \leftarrow \cdots
$$

in A with $d_{n} d_{n+1}=0$. (We often set $d_{0}=0: X_{0} \rightarrow 0$ and write $d$ instead of $d_{n}$.) A map $f: X \rightarrow X^{\prime}$ of DG-objects is a map of diagrams; that is, a family of maps $\left\{f_{n}: X_{n} \rightarrow X_{n}^{\prime} \mid n=0,1,2, \cdots\right\}$ of $\mathbf{A}$ such that $d_{n}^{\prime} f_{n}=f_{n-1} d_{n}$ for every $n$. With these maps as morphisms, and the evident composition of maps, the DG-objects for $\mathbf{A}$ form an abelian category $\mathrm{DG}(\mathrm{A})$; for example, a sequence of maps $X \rightarrow Y \rightarrow Z$ is exact 
in DG(A) precisely when every sequence $X_{n} \rightarrow Y_{n} \rightarrow Z_{n}$ is exact in A. Again, $\operatorname{DG}(\mathbf{A})$ can be described as a functor category. Take $\mathbf{L}$ to be the category with objects all natural numbers and with groups of morphisms

$$
\text { hom } \begin{aligned}
\operatorname{hon}(m) & =\text { the infinite cyclic group generated by } 1_{m} \text { if } m=n ; \\
& =\text { the infinite cyclic group generated by } d_{n+1} \text { if } m=n+1, \\
& =0 \text { otherwise }(m \neq n+1, n) .
\end{aligned}
$$

Then an additive functor $\mathbf{L} \rightarrow \mathbf{A}$ is just a DG-object for $\mathbf{A}$.

Observe the inclusion of (abelian) categories

$$
A \subset \mathrm{G}(\mathrm{A}) \subset \mathrm{DG}(\mathrm{A})
$$

where each object $A$ is interpreted as a graded object with $A_{n}=0$ for $n \neq 0$ and $A_{0}=A$, while each graded object is interpreted as a DGobject with zero differential.

For example, let $\mathbf{A}$ be the category of abelian groups. Then a DGobject for $\mathbf{A}$ is just a chain complex $X$ of abelian groups, while a DGmap is a chain transformation. To get the homology of $X$, first take the kernel $C_{n}$ of $d_{n}: X_{n} \rightarrow X_{n-1}$. Since $d d=0, C_{n} \subset d X_{n+1}$; the $n$th homology group is defined as the quotient $H_{n}(X)=C_{n} / d X_{n+1}$-in geometric language, as cycles modulo boundaries.

This can be done for any A. In the DG-object $X$, let $k_{n}: C_{n} \rightarrow X_{n}$ be the kernel of $d_{n}$. Then $d_{n} d_{n+1}=0$ implies that $d_{n+1}$ factors through $k_{n}$ as $d=k e$, for some $e$. The cokernel of $e$ has a codomain which is defined to be the homology $H_{n}(X)$, as in the diagram

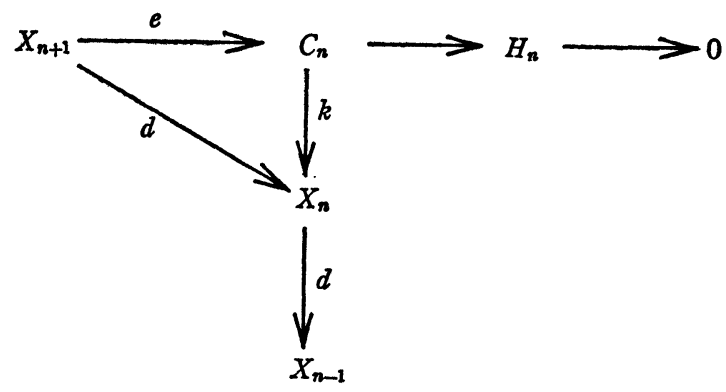

with row right exact and column left exact. It is readily verified that $H(X)=\left\{H_{n}(X) \mid n=0,1,2, \cdots\right\}$ is an additive functor $H: \mathrm{DG}(\mathbf{A})$ $\rightarrow \mathrm{G}(\mathrm{A})$.

This homology functor is not exact. However, a standard construction yields a "connecting morphism" as follows. If $E: X \frown,^{f} Y \rightarrow{ }^{\circ} Z$ is a short exact sequence of DG-objects for $\mathbf{A}$, its connecting morphism 
is a morphism $E_{*}: H(Z) \rightarrow H(X)$ of graded objects, with degree -1 , such that the following triangle

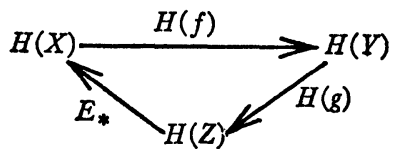

is exact at each vertex. This $E_{*}$ may be defined as that morphism whose "graph" is the composite of the following (additive) relations (Homology, II.6):

$$
H(Z) \longrightarrow Z \stackrel{g^{-1}}{\longrightarrow} Y \stackrel{d}{\longrightarrow} Y \stackrel{f^{-1}}{\longrightarrow} X \longrightarrow H(X) .
$$

Our graded objects have been graded by the positive integers $(n=0,1,2, \cdots)$; according to Bourbaki, "positive" means "nonnegative." One may also consider objects $\cdots, X_{-2}, X_{-1}, X_{0}, X_{1}, \cdots$ graded by all the integers $Z$ and the corresponding differential $Z$ graded objects $X$; the homology of such a $\mathrm{DG}_{Z}$-object is a $Z$-graded object.

For DG-objects $X$ and $Y$ of $\mathrm{A}$ the $Z$-graded abelian group

$$
\operatorname{Hom}_{n}(X, Y)=\prod_{k} \operatorname{hom}_{A}\left(X_{k}, Y_{n+k}\right), \quad n=0, \pm 1, \cdots,
$$

where $\Pi$ denotes the cartesian product over all $k \in Z$, has as elements $f$ of degree $n$ the morphisms $f=\left\{f_{k}: X_{k} \rightarrow Y_{k+n}\right\}: X \rightarrow Y$ of degree $n$ between the graded objects $X$ and $Y$. Define a differential $D: \mathrm{Hom}_{n} \rightarrow \mathrm{Hom}_{n-1}$ from the differentials $d_{Y}$ of $Y$ and $d_{X}$ of $X$ by

$$
(D f)_{k}=d_{Y} f_{k}+(-1)^{n+1} f_{k-1} d_{X}: X_{k} \rightarrow Y_{k+n-1} \text {. }
$$

These definitions yield a $D_{Z}$-abelian group $\operatorname{Hom}(X, Y)$ which is a functor

$$
\text { Hom: } \mathrm{DG}(\mathrm{A})^{\text {op }} \times \mathrm{DG}(\mathrm{A}) \rightarrow \mathrm{DG}_{\mathbf{Z}}(\mathrm{Ab}),
$$

additive and left exact in each argument separately (Homology, II.3, VI.7). We write Hom, with a capital " $\mathrm{H}$," for this $\mathrm{DG}_{\mathbf{z}}$-group, which consists of morphisms $f: X \rightarrow Y$ of graded objects, to distinguish it from $\operatorname{hom}(X, Y)$, with the lower case " $\mathrm{h}$," which is the abelian group of maps $f: X \rightarrow Y$ (of degree zero) of DG-objects. Note that the cycles of degree zero in $\mathrm{Hom}_{0}$ are all those $f$ of degree zero which satisfy $D f=0$; by (5), these are exactly the maps $X \rightarrow Y$ of DG-objects (the chain transformations). A homotopy $s: f \simeq g$ between two such maps $f, g: X \rightarrow Y$ is defined to be an element $s \in \operatorname{Hom}_{1}(X, Y)$ with $D s=f-g$. By (5), with $n=1$, this states that 


$$
d_{Y} s_{k}+s_{k-1} d_{X}=f_{k}-g_{k}, \quad k=0,1, \cdots .
$$

This is the usual definition of a "chain homotopy" betwen two chain transformations.

In Chapter IV we shall use resolutions of an object $A$ of $\mathbf{A}$. Regard $A$, as in (1), as a trivially graded DG-object. Then a DG-object $X$ over $A$ is defined to be a DG-object $X$ together with a map $\epsilon: X \rightarrow A$ of such objects; in other words, it is a diagram

$$
A \stackrel{\epsilon}{\leftarrow} X_{0} \stackrel{d}{\leftarrow} X_{1} \stackrel{d}{\leftarrow} X_{2} \stackrel{d}{\leftarrow} \ldots
$$

in A with $d d=0$ and $\epsilon d=0$. The map $\epsilon$ induces $\epsilon_{*}: H_{0}(X) \rightarrow A$.

A contraction (or a contracting homotopy) for $\epsilon: X \rightarrow A$ consists of a map $s_{-1}: A \rightarrow X$ of DG-objects with $\epsilon s_{-1}=1_{A}$ and a homotopy $s: 1_{0} \simeq s_{-1} \epsilon$. Thus a contraction gives a diagram

$$
A \underset{s_{-1}}{\stackrel{\epsilon}{\leftrightarrows}} X_{0} \underset{s_{0}}{\stackrel{d}{\leftrightarrows}} X_{1} \underset{s_{1}}{\stackrel{d}{\leftrightarrows}} X_{2} \leftrightarrows \cdots
$$

in $A$ such that $d d=0, \epsilon d=0$, and

(9) $\quad \epsilon s_{-1}=1, \quad s_{-1} \epsilon+d s_{0}=1_{0}, \quad s_{n} d+d s_{n+1}=1_{n+1}, \quad n=0,1, \cdots$

The presence of $s$ insures that $\epsilon_{*}: H_{0}(X) \cong A$ and $H_{n}(X)=0$ for $n>0$.

Chapter III. Types of Algebras

15. Coherence. For many different sorts of algebras-graded, bigraded, or differential graded - the formal properties can be developed in common, as a theory of algebras over a suitable type of category.

A category with a multiplication is a category $\mathbf{C}$ together with a covariant bifunctor $\otimes: \mathbf{C} \times \mathbf{C} \rightarrow \mathbf{C}$; this bifunctor will be written between its arguments, so that morphisms $f: A \rightarrow A^{\prime}$ and $g: B \rightarrow B^{\prime}$ give a morphism $f \otimes g: A \otimes B \rightarrow A^{\prime} \otimes B^{\prime}$ of $\mathrm{C}$. The conditions that $\otimes$ be a bifunctor read

$$
1_{A} \otimes 1_{B}=1_{A \otimes B}, \quad\left(f^{\prime} f\right) \otimes\left(g^{\prime} g\right)=\left(f^{\prime} \otimes g^{\prime}\right)(f \otimes g),
$$

the latter whenever the composites $f^{\prime} f$ and $g^{\prime} g$ are defined. Assume that there are given natural isomorphisms ("associativity" and "commutativity"):

$$
\begin{aligned}
& a=a(A, B, C): A \otimes(B \otimes C) \cong(A \otimes B) \otimes C, \\
& c=c(A, B): A \otimes B \cong B \otimes A .
\end{aligned}
$$

We may also assume that $\mathbf{C}$ has a fixed object $K$, called the ground object, which acts as an identity for the multiplication $\otimes$, in the sense that there are given natural isomorphisms (left and right "identity" isomorphisms) 


$$
e=e(A): K \otimes A \cong A, \quad e^{\prime}: A \otimes K \cong A .
$$

For example, let $\mathbf{C}$ be any category with finite products and a terminal element. The product $A \Pi B$ of two objects of $\mathbf{C}$ then provides such a multiplication in $\mathrm{C}$. Since both triple products $A \Pi(B \Pi C)$ and $(A \Pi B) \Pi C$ have co-universal properties, relative to their projections on $A, B$, and $C$, there is a natural isomorphism for associativity, and similarly for commutativity. If $K$ is a terminal element for $\mathbf{C}$, then each object $C$, with $1: C \rightarrow C$ and the unique map $C \rightarrow K$ as projections, provides a product $C=C \Pi K$, and hence identity isomorphisms $e$ and $e^{\prime}$.

Again, for example, let $K$ be a commutative ring (with identity element) and $K$-Mod the category of all $K$-modules. The tensor product $A \otimes B$ of two $K$-modules is a $K$-module, and so provides a multiplication for $K$-Mod. Associativity and commutativity isomorphisms are given by the familiar maps

$$
x \otimes(y \otimes z) \rightarrow(x \otimes y) \otimes z, \quad x \otimes y \rightarrow y \otimes x .
$$

Consider now iterated associativity diagrams, such as the pentagon

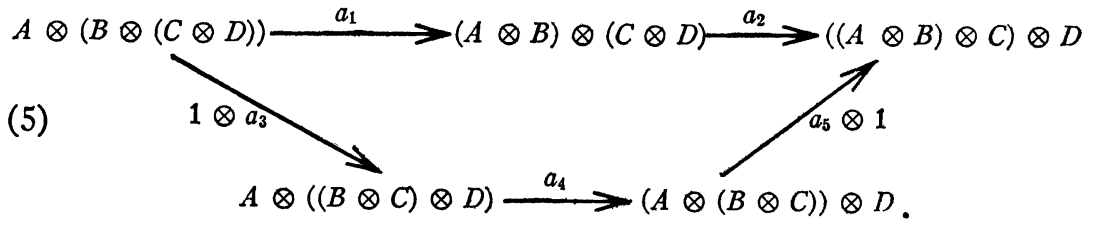

Here each $a_{i}$ is an evident "instance" of the given natural associativity isomorphism; for example, $a_{2}=a(A \otimes B, C, D)$. In the familiar associativity maps, such as (4) for modules, this diagram is clearly commutative, though it would not be so were (4) replaced by the different natural isomorphism $x \otimes(y \otimes z) \rightarrow-(x \otimes y) \otimes z$. With Bénabou [1], we say that an associativity isomorphism $a$ is coherent if every diagram built up from such instances of $a$ is commutative. Here "every diagram" (see MacLane [78] for details) means every diagram whose vertices represent iterates of the tensor product functor and whose edges are tensor products of identity maps and instances of the associativity $a$; while an "instance" of $a$ is one formed from $a$ by substitutions (as in $\$ 5$ ) of iterates of the tensor product functor.

Thus coherence involves an infinite number of conditions which can be reduced to a single condition; Stasheff [97] by geometric means and MacLane [78] by algebraic ones prove

THEOREM 15.1. In a category $\mathbf{C}$ with a multiplication, a natural associ- 
ativity isomorphism is coherent if and only if all the pentagonal diagrams (5) are commutative.

Here the naturality of $a$ is essential to this theorem; it yields the commutativity of diagrams such as

$$
\begin{gathered}
A \otimes[(B \otimes(C \otimes D)) \otimes E] \stackrel{a}{\rightarrow}[A \otimes(B \otimes(C \otimes D))] \otimes E \\
\downarrow 1 \otimes(a \otimes 1) \\
\downarrow \otimes[((B \otimes C) \otimes D) \otimes E] \stackrel{a}{\rightarrow}[A \otimes((B \otimes C) \otimes D)] \otimes E .
\end{gathered}
$$

The full diagram of all commutativities for products of five objects forms a polyhedral subdivision of a 3-cell, and similarly in higher dimensions (Stasheff).

Associativity and commutativity isomorphisms $a$ and $c$ will be called jointly coherent if and only if every diagram built from instances of $a$ and $c$ is commutative. Again, this coherence requirement can be reduced to a finite number of conditions; Epstein [31] and MacLane [78] prove

THEOREM 15.2. Associativity and commutativity isomorphisms a and $c$ are jointly coherent if and only if the pentagonal diagrams (5) and the two following diagrams are always commutative:

$$
\begin{aligned}
& A \otimes B \\
& \underbrace{c}_{\swarrow c} B \otimes A, \\
& A \otimes B \\
& A \otimes(B \otimes C) \stackrel{a}{\longrightarrow}(A \otimes B) \otimes C \stackrel{c}{\longrightarrow} C \otimes(A \otimes B) \\
& \downarrow 1 \otimes C \\
& A \otimes(C \otimes B) \stackrel{a}{\longrightarrow}(A \otimes C) \otimes B \stackrel{c \otimes 1}{\longrightarrow}(C \otimes A) \otimes B .
\end{aligned}
$$

These coherence conditions will insure, for example, that for four given objects there is a unique natural isomorphism

$$
m:(A \otimes B) \otimes(C \otimes D) \cong(A \otimes C) \otimes(B \otimes D) ;
$$

indeed, in the familiar cases this is the "middle four interchange" which acts on elements as $(a \otimes b) \otimes(c \otimes d) \rightarrow(a \otimes c) \otimes(b \otimes d)$.

Finally, sufficient joint coherence conditions for $a, c$, and left and right identity isomorphisms $e$ and $e^{\prime}$ are the three diagrams of Theorem 15.2 plus the condition $e^{\prime}=e c: A \otimes K \rightarrow A$ and the two conditions 


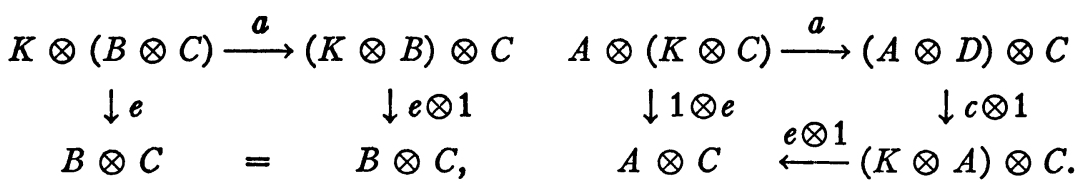

Kelly [61] has studied the independence of these coherence conditions.

16. Tensored categories. We now define the type of category (examples: $K$-modules, bigraded $K$-modules) over which a formal theory of algebras may be developed. A tensored category $\mathbf{D}$ will be an abelian category equipped with a selected ground object $K$ and a bifunctor $\otimes: \mathbf{D} \times \mathbf{D} \rightarrow \mathbf{D}$ which is additive and cokernel preserving (=right exact) in each argument separately. Moreover, there are given natural isomorphisms $a$ (associativity), $c$ (commutativity) and $e, e^{\prime}$ (left and right identity to $K$ ) which are jointly coherent, in the sense just discussed.

If $D$ is a tensored category, so is the category $G(D)$ of graded objects for $\mathbf{D}$. Indeed, if $X$ and $Y$ are graded objects for $\mathbf{D}$, their tensor product is defined to be the graded object with

$$
(X \otimes Y)_{n}=\sum_{p+q=n} X_{p} \otimes Y_{q} \quad\left(\sum=\text { iterated biproduct }\right),
$$

with the evident tensor product of maps. The ground object of $G(D)$ is the trivially graded object $K$. The natural isomorphisms $a, e$, and $e^{\prime}$ for $\mathrm{G}(\mathrm{D})$ are evident, while the "commutation" $c_{n}:(X \otimes Y)_{n}$ $\rightarrow(Y \otimes X)_{n}$ is the map $\sum X_{p} \otimes Y_{q} \rightarrow \sum Y_{q} \otimes X_{p}$ of biproducts whose only nonzero components are the $(-1)^{p q} c\left(X_{p}, Y_{q}\right): X_{p} \otimes Y_{q} \rightarrow Y_{q} \otimes X_{p}$ for each $p+q=n$. This exhibits the sign commutation rule: transposition of objects or symbols of degrees $p$ and $q$ introduces a sign $(-1)^{p q}$. A similar sign occurs in the definition of the tensor products of morphisms $f: X \rightarrow X^{\prime}$ and $g: Y \rightarrow Y^{\prime}$ of given degrees; the morphism $f \otimes g: X \otimes Y \rightarrow X^{\prime} \otimes Y^{\prime}$ has degree the sum of $\operatorname{deg} f$ and $\operatorname{deg} g$, and $(f \otimes g)_{n}$ is the map of biproducts whose only nonzero components are

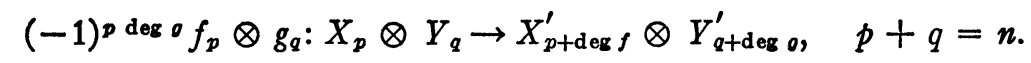

Similarly, if $\mathbf{D}$ is a tensored category, so is the category $D G(D)$. For two DG-objects $X$ and $Y$, the grading of $X \otimes Y$ is defined by (1) above, while the differential $d:(X \otimes Y)_{n} \rightarrow(X \otimes Y)_{n-1}$ is the map of biproducts whose only nonzero components are

(3) $d \otimes 1: X_{p} \otimes Y_{q} \rightarrow X_{p-1} \otimes Y_{q}, \quad(-1)^{p}(1 \otimes d): X_{p} \otimes Y_{q} \rightarrow X_{p} \otimes Y_{q-1}$

for $p+q=n$. This agrees with the sign rule (2) and with the usual formula 


$$
d(x \otimes y)=d x \otimes y+(-1)^{\operatorname{deg} x} x \otimes d y
$$

for the tensor product of chain complexes (Homology, V.9).

17. Algebras. Consider the usual data determining an algebra $\Lambda$ over a commutative ring $K$. First, $\Lambda$ is a $K$-module; the product $a b$ of two elements $a$ and $b$ is bilinear, hence may be regarded as the map $p: \Lambda \otimes \Lambda \rightarrow \Lambda$ of $K$-modules with $p(a \otimes b)=a b$, while the identity element 1 of $\Lambda$ provides a unit map $u: K \rightarrow \Lambda$ of $K$-modules via $k \rightarrow k 1$. The further conditions (associativity, etc.) may then be stated in the tensored category $K$-Mod. Exactly the same process applies to other types of algebras.

To state this process generally, let $\mathbf{D}$ be a tensored category with ground object $K$. A D-algebra is an object $\Lambda$ of $\mathbf{D}$ together with two morphisms $p=p_{\Delta}: \Lambda \otimes \Lambda \rightarrow \Lambda$ and $u=u_{\Lambda}: K \rightarrow \Lambda$ of $\mathrm{D}$ such that the diagrams

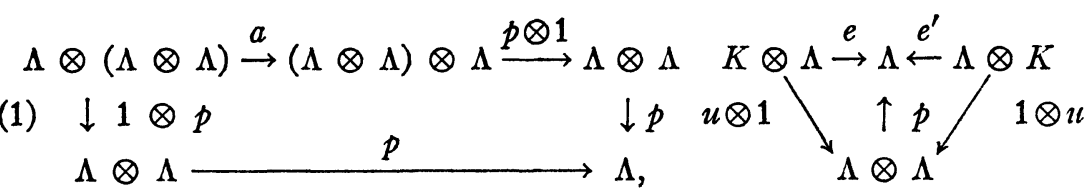

commute, for $a, e$, and $e^{\prime}$ as in (15.1) and (15.2). The first diagram states that the product $p$ is associative, the second that it has a left and right "identity element." The ground object $K$ of $\mathbf{D}$ is a $\mathbf{D}$ algebra with product $e_{K}=e_{K}^{\prime}: K \otimes K \rightarrow K$. A morphism of D-algebras $\lambda: \Lambda \rightarrow \Lambda^{\prime}$ is a morphism of $D$ such that

(2) $\lambda p_{\Lambda}=p_{\Lambda^{\prime}}(\lambda \otimes \lambda): \Lambda \otimes \Lambda \rightarrow \Lambda^{\prime}$ and $\lambda u_{\Lambda}=u_{\Lambda^{\prime}}: K \rightarrow \Lambda^{\prime}$.

The D-algebras constitute a category $\operatorname{Alg}(\mathrm{D})$ with a ground object $K$. An augmented $D$-algebra is a morphism $\epsilon: \Lambda \rightarrow K$ in this category.

The opposite $\Lambda^{\mathrm{op}}$ of a $D$-algebra $\Lambda$ is the object $\Lambda$ equipped with the composite map $p_{\Lambda} \circ c: \Lambda \otimes \Lambda \rightarrow \Lambda \otimes \Lambda \rightarrow \Lambda$ as product. For example (with $\mathrm{Ab}$ the category of abelian groups):

Ab-algebras are rings (with an identity, as always),

$K$-Mod-algebras are algebras over the ground ring $K$,

$\mathrm{G}(K$-Mod)-algebras are graded algebras over $K$,

$\mathrm{DG}(K$-Mod)-algebras are differential graded algebras over $K$, and so on ( $Z$-graded algebras, bigraded algebras, etc.). A D-algebra $\Lambda$ is commutative if $c p=p: \Lambda \otimes \Lambda \rightarrow \Lambda$. Thus, in view of the sign rules, commutativity for a graded algebra over $K$ means, for the product of elements, that $a b=(-1)^{(\operatorname{deg} a)(\operatorname{deg} b)} b a$.

The tensor product of two D-algebras $\Lambda^{\prime}$ and $\Lambda^{\prime \prime}$ is the object $\Lambda^{\prime} \otimes \Lambda^{\prime \prime}$ of $\mathrm{D}$ with unit map the composite $K \rightarrow K \otimes K \rightarrow \Lambda^{\prime} \otimes \Lambda^{\prime \prime}$ and product map the composite 


$$
\left(\Lambda^{\prime} \otimes \Lambda^{\prime \prime}\right) \otimes\left(\Lambda^{\prime} \otimes \Lambda^{\prime \prime}\right) \stackrel{m}{\rightarrow}\left(\Lambda^{\prime} \otimes \Lambda^{\prime}\right) \otimes\left(\Lambda^{\prime \prime} \otimes \Lambda^{\prime \prime}\right) \stackrel{p^{\prime} \otimes p^{\prime \prime}}{\rightarrow} \Lambda^{\prime} \otimes \Lambda^{\prime \prime},
$$

where $m$ is the middle four interchange (15.5). This tensor product is associative and satisfies $K \otimes \Lambda \cong \Lambda \cong \Lambda \otimes K$. Since the tensor product of algebras may be regarded as a bifunctor $A \lg (\mathbf{D}) \times \operatorname{Alg}(\mathbf{D}) \rightarrow A \lg (\mathbf{D})$, the category $\operatorname{Alg}(\mathrm{D})$ of $\mathbf{D}$-algebras is a multiplicative category with ground object $K$ and associativity and left and right identity isomorphisms which are readily seen to be coherent (in consequence of the coherence of $\mathbf{D}$ ).

With elements, the tensor product algebra $\Lambda^{\prime} \otimes \Lambda^{\prime \prime}$ can be characterized as the "universal" algebra in which the elements of $\Lambda^{\prime}$ and $\boldsymbol{\Lambda}^{\prime \prime}$ commute. This can be stated without elements. For fixed $\mathbf{D}$ algebras $\Lambda^{\prime}$ and $\Lambda^{\prime \prime}$, consider the category of all those diagrams of $D$ algebras

$$
\Lambda^{\prime} \stackrel{\lambda^{\prime}}{\longrightarrow} \Omega \stackrel{\lambda^{\prime \prime}}{\longleftarrow} \Lambda^{\prime \prime}
$$

for which the following diagram is commutative

$$
\begin{gathered}
\Lambda^{\prime} \otimes \Lambda^{\prime \prime} \stackrel{\lambda^{\prime} \otimes \lambda^{\prime \prime}}{\longrightarrow} \Omega \otimes \Omega \stackrel{p}{\longrightarrow} \Omega \\
\downarrow \downarrow \\
\Lambda^{\prime \prime} \otimes \Lambda^{\prime} \stackrel{\lambda^{\prime \prime} \otimes \lambda^{\prime}}{\longrightarrow} \Omega \otimes \Omega \stackrel{p}{\longrightarrow}
\end{gathered}
$$

The tensor product $\Omega=\Lambda^{\prime} \otimes \Lambda^{\prime \prime}$, equipped with the two composite maps

$$
\Lambda^{\Lambda^{\prime} \stackrel{e^{-1}}{\longrightarrow}} \Lambda^{\prime} \otimes K \stackrel{1 \otimes u^{\prime \prime}}{\longrightarrow} \Lambda^{\prime} \otimes \Lambda^{\prime \prime}, \quad \Lambda^{\prime \prime} \rightarrow K \otimes \Lambda^{\prime \prime} \rightarrow \Lambda^{\prime} \otimes \Lambda^{\prime \prime},
$$

provides an initial object in this category of diagrams.

In the category $\operatorname{Alg}_{\mathrm{c}}(\mathbf{D})$ of commutative $\mathbf{D}$-algebras, this diagram makes $\Lambda^{\prime} \otimes \Lambda^{\prime \prime}$ a coproduct and gives a commutativity $\Lambda^{\prime} \otimes \Lambda^{\prime \prime}$ $\cong \Lambda^{\prime \prime} \otimes \Lambda^{\prime}$ which is coherent (jointly with $a, e$, and $e^{\prime}$ ).

This "diagrammatic" description of algebras is especially useful for the dual notion, that of a coalgebra. A D-coalgebra $C$ is an object of $\mathrm{D}$ together with two morphisms, a coproduct $q: C \rightarrow C \otimes C$ and a counit $v: C \rightarrow K$ such that the duals of (1) hold, that is:

$$
\begin{aligned}
a(1 \otimes q) q & =(q \otimes 1) q: C \rightarrow(C \otimes C) \otimes C, \\
e^{\prime-1}(1 \otimes v) q & =1=e^{-1}(v \otimes 1) q: C \rightarrow C .
\end{aligned}
$$

Morphisms and tensor products of coalgebras are defined correspondingly.

A D-hopf algebra $V$ (Milnor-Moore [79]) is an object $V$ of $\mathbf{D}$ which is both an algebra (with $p, u$, and (1)) and a coalgebra (with $q, v$, and (3)) such that $u: K \rightarrow V$ is a morphism of $\mathbf{D}$-coalgebras, while 
$v: V \rightarrow K$ and $q: V \otimes V \rightarrow V$ are both morphisms of $\mathbf{D}$-algebras. This last condition states that the following diagram commutes

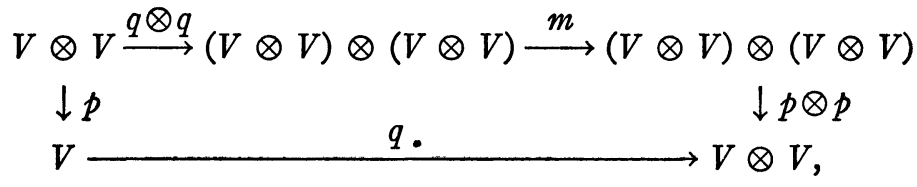

with $m$ the middle four interchange. This condition may also be read: $p$ is a morphism of coalgebras.

18. Modules over algebras. Let $\Lambda$ be a $\mathrm{D}$-algebra. A left $\Lambda$-module $A$ is an object $A$ of $\mathrm{D}$ together with a morphism $p_{\Lambda}: \Lambda \otimes A \rightarrow A$ of $\mathrm{D}$, called "left operation by $\Lambda$," such that

$$
\begin{aligned}
p_{A}\left(p_{\Lambda} \otimes 1\right) a & =p_{A}\left(1 \otimes p_{A}\right): \Lambda \otimes(\Lambda \otimes A) \rightarrow A, \\
p_{A}\left(u_{\Delta} \otimes 1\right) & =e: K \otimes A \rightarrow A,
\end{aligned}
$$

much as in (17.1). For elements, the first equation would state that left operation is associative, the second that left operation by the identity element of $\Lambda$ is the identity operation. A map $f: A \rightarrow B$ of left $\Lambda$-modules is a morphism of $\mathrm{D}$ such that $f p_{A}=p_{B}(1 \otimes f): \Lambda \otimes A \rightarrow B$. The composite of $\Lambda$-module morphisms is again such. If $k: D \rightarrow A$ is a kernel of $f$ in $\mathbf{D}$, as in (11.2), then

$$
f p_{A}(1 \otimes k)=p_{B}(1 \otimes f)(1 \otimes k)=p_{B}(1 \otimes f k)=0: \Lambda \otimes D \rightarrow B ;
$$

therefore $p_{A}(1 \otimes k)$ factors uniquely through the kernel $k$ as $p_{A}(1 \otimes k)$ $=k p^{\prime}$, for some morphism $p^{\prime}: \Lambda \otimes D \rightarrow D$. With this morphism $p^{\prime}$ as left operation, $D$ is a left $\Lambda$-module and $k$ a map of left $\Lambda$-modules. Similarly the image (and also the cokernel) of $f$ has a left $\Lambda$-module structure, and a sequence of $\Lambda$-module maps is exact if and only if it is exact as a sequence of morphisms of $\mathbf{D}$. With such arguments one proves

Proposition 18.1. If $\Lambda$ is an algebra for a tensored category $\mathbf{D}$, the left $\Lambda$-modules and their maps constitute an abelian category $\Lambda$-Mod(D).

This category has a "ground object;" the algebra $\Lambda$ itself regarded as a left $\Lambda$-module, with left operation given by the product in $\Lambda$. If $A$ is a left $\Lambda$-module and $D$ any object of $\mathrm{D}$, then $D \otimes A$ is a left $\Lambda$-module, with left operation by $\Lambda$ given by the composite

$$
\begin{aligned}
\Lambda \otimes(D \otimes A) & \rightarrow(\Lambda \otimes D) \otimes A \rightarrow(D \otimes \Lambda) \otimes A \rightarrow D \otimes(\Lambda \otimes A) \\
& \rightarrow D \otimes A ;
\end{aligned}
$$

we call this the $\Lambda$-module structure of $D \otimes A$ inherited from $A$. In this 
diagrammatic definition the first three maps are given by the isomorphisms $a, c$, and $a^{-1}$; by the coherence assumption, it suffices to write this composite as the natural isomorphism $\Lambda \otimes(D \otimes A)$ $\rightarrow D \otimes(\Lambda \otimes A)$.

Right $\Lambda$-modules $R$ are treated similarly, via a right operation $R \otimes \Lambda \rightarrow R$. Each right $\Lambda$-module may also be regarded as a left $\Lambda^{\mathrm{op}}$-module, with operation $\Lambda^{\mathrm{op}} \otimes R \rightarrow \rightarrow^{e} R \otimes \Lambda \rightarrow R$. If $\Lambda$ and $\Sigma$ are two D-algebras, a $\Lambda-\Sigma$ bimodule $B$ is a left- $\Lambda$ and right- $\Sigma$ module such that the composite $\Lambda \otimes B \otimes \Sigma \rightarrow \Lambda \otimes B \rightarrow B$ is the same as the composite $\Lambda \otimes B \otimes \Sigma \rightarrow B \otimes \Sigma \rightarrow B$; such a module may also be regarded as a left $\left(\Lambda \otimes \Sigma^{\mathrm{op}}\right)$-module. Other formal properties are as usual.

Since each left $\Lambda$-module is, to begin with, an object of $D$, there is a forgetful functor $G: \Lambda-\operatorname{Mod}(D) \rightarrow D$. On the other hand, for each $D$ in $\mathrm{D}$, the tensor product $\Lambda \otimes D$ is a left $\Lambda$-module with left operation inherited from $\Lambda$; this gives a functor $F: D \rightarrow \Lambda-\operatorname{Mod}(D)$, with $F(f)=1 \otimes f$.

TheOREM 18.2. For $\mathrm{D}$ a tensored category and $\Lambda$ a $\mathrm{D}$-algebra, the functor $F$ with $F(D)=\Lambda \otimes D$ is an adjoint to the forgetful functor $G$ : $\Lambda-\operatorname{Mod}(\mathbf{D}) \rightarrow \mathbf{D}$ from left $\Lambda$-modules to objects of $\mathbf{D}$. Moreover, $G$ is additive and exact, while $F$ is additive and right exact.

PRoof. The stated exactness is immediate from the assumed right exactness of the tensor product in $\mathbf{D}$. For the rest, it suffices to construct a universal $e: I \rightarrow G F$. For each $D$, take $e_{D}$ to be the composite $D \rightarrow K \otimes D \rightarrow{ }^{u} \otimes^{1} \Lambda \otimes D$. Then if $C$ is any $\Lambda$-module and $f: D \rightarrow G(C)$ a morphism of $\mathrm{D}$, we may define $\alpha: \Lambda \otimes D \rightarrow C$ as the composite $\Lambda \otimes D$ $\rightarrow{ }^{1{ }^{f}} \Lambda \otimes C \rightarrow C$; this is a map of left $\Lambda$-modules, satisfies $G(\alpha) e_{D}=f$, and is the only such $\alpha$; hence we have a universal junction, as required.

Let $R$ be a right $\Lambda$-module and $A$ a left $\Lambda$-module. Their tensor product $R \otimes_{\Delta} A$ over $\Lambda$ is an object of $\mathrm{D}$, defined as the cokernel object of the map $h=\left(p_{R} \otimes 1\right) a-1 \otimes p_{A}$, as displayed in the right exact sequence

(2) $R \otimes(\Lambda \otimes A) \stackrel{h}{\rightarrow} R \otimes A \stackrel{t}{\rightarrow} R \otimes_{\Lambda} A \rightarrow 0, \quad h=\left(p_{R} \otimes 1\right) a-1 \otimes p_{A}$.

This corresponds directly to the usual definition with elements, where $R \otimes_{\Delta} A$ is obtained from $R \otimes_{K} A$ by the identifications $r \lambda \otimes a=r \otimes \lambda a$ for $\lambda \in \Lambda, r \in R$, and $a \in A$. Since $h$ is natural, any map $f: A \rightarrow A^{\prime}$ of left $\Lambda$-modules induces a morphism $f_{*}=1 \otimes_{\Lambda} f: R \otimes_{\Lambda} A \rightarrow R \otimes_{\Lambda} A^{\prime}$. A similar argument for maps of $R$ shows that $\otimes_{\Delta}$ is a bifunctor, additive in each argument separately. Since the functor $\otimes$ is right exact in $\mathrm{D}$, each right exact sequence $A \nrightarrow B \longrightarrow C$ of left $\Lambda$-modules yields a 
$3 \times 3$ commutative diagram with rows the $A, B$, and $C$ cases of (2); the $3 \times 3$ Lemma (Homology, XII.3.3) then proves that $\otimes_{A}$ is right exact in its second argument. It is similarly right exact in its first argument. This discussion shows that the formal theory of the tensor product works over a tensored category $\mathbf{D}$ just as it does for modules over an ordinary algebra with honest elements. Moreover, our discussion applies also to other cases; for example, it will give all the usual properties for modules over a differential graded algebra without any extra attention to differential or grading.

Now let $\Lambda$ be a commutative $\mathrm{D}$-algebra and $A$ a left $\Lambda$-module. Then the morphism $p_{A}: \Lambda \otimes A \rightarrow A$ giving the left operation of $\Lambda$ on $A$ is also a map of $\Lambda$-modules, when $\Lambda \otimes A$ has the left $\Lambda$-module structure inherited from $A$. The left $\Lambda$-module $A$ can also be regarded as a right $\Lambda$-module, with right operation given by the composite

$$
A \otimes \Lambda \stackrel{c}{\rightarrow} \Lambda \otimes A \stackrel{p_{A}}{\rightarrow} A .
$$

Moreover, the tensor product over $\Lambda$ of two $\Lambda$-modules is again a $\Lambda$-module. Specifically, the commutativity of $\Lambda$ shows that the morphism $h$ of (2) is a map of the left $\Lambda$-module structures inherited from $A$; hence the cokernel $R \otimes{ }_{\Lambda} A$ acquires a left $\Lambda$-module structure, while the $t$ of (2) is a map of left $\Lambda$-modules. By a similar argument, $h$ is a map for the right $\Lambda$-module structures inherited from $R$, so that $R \otimes_{\Lambda} A$ is a right $\Lambda$-module and thence, as in (3), a left $\Lambda$ module. The form of the map $h$ now implies that these two left $\Lambda$ module structures on $R \otimes_{\Delta} A$-that inherited from $R$, and that from $A$-are in fact the same $\Lambda$-module structure. With this tensor product, we obtain

Proposition 18.3. If $\mathbf{D}$ is a tensored category, so is the category of modules over a commutative $\mathrm{D}$-algebra $\Lambda$.

\section{Chapter IV. Relative Homological Algebra}

19. Resolvent pairs. This chapter indicates how abelian categories and adjoint functors may be used to organize the treatment of derived functors in homological algebra. We restrict ourselves to "relative" homological algebra and start with a pair of adjoint functors. Explicitly, a resolvent pair $\mathbf{R}$ of categories is given by the data

$$
G: \mathbf{A} \rightarrow \mathbf{M}, \quad F: \mathbf{M} \rightarrow \mathbf{A}, \quad u: I \rightarrow G F,
$$

where $\mathbf{A}$ and $\mathbf{M}$ are abelian categories, $G$ is an additive and faithful functor which takes epics in $\mathbf{A}$ to epics in $\mathbf{M}, F$ is a functor, and $u$ is a universal junction of $F$ to $G$; it follows (Theorem 13.1) that $G$ 
is exact, that $F$ is additive and right exact, and that $F$ is an adjoint of $G$. We shall write $\alpha: A \rightarrow B, \beta, \cdots$ for morphisms of $\mathbf{A}$ and $f: L \rightarrow M, g, \cdots$ for those of $\mathbf{M}$.

There are a number of examples of this situation, as follows:

A pair of rings. Let $S$ be a subring of the ring $R$, with the same identity element as $R$. Take

$\mathrm{A}=$ left $R$-modules, $\mathbf{M}=$ left $S$-modules, $F(M)=R \otimes_{S} M, u_{M}(m)=1 \otimes m$, with $G: \mathbf{A} \rightarrow \mathbf{M}$ the evident forgetful functor (regard each left $R$ module just as a left $S$-module).

A tensored category. Let $\mathrm{D}$ be a tensored category and $\Lambda$ a $\mathrm{D}$-algebra. Take

(2) $\mathrm{A}=$ left $\Lambda$-modules, $\mathrm{M}=\mathrm{D}, G$ the forgetful functor, $F(M)=\Lambda \otimes M$;

Theorem 18.2 gives a universal junction $u: I \rightarrow G F$. This example includes all the following more explicit cases:

A multiplicative group $G$. Let " $G$-module" mean "left module over the integral group ring $Z(G)$." Take $\Lambda=Z(G)$,

$$
\mathbf{A}=G \text {-modules, } \mathbf{M}=\text { abelian groups, } G \text { and } F \text { as in (2). }
$$

This may also be regarded as a pair of rings $S=Z \subset R=Z(G)$.

An algebra. Let $\Lambda$ be an algebra over the commutative ground ring $K$. Take

$\mathbf{A}=$ left $\Lambda$-modules, $\mathbf{M}=K$-modules, $G$ and $F$ as in (2), $u(m)=1 \otimes m$.

An algebra with bimodules. Let $\Gamma$ be a $K$-algebra. Take

$\mathrm{A}=\Gamma$-bimodules, $\mathbf{M}=$ right $\Gamma$-modules, $G$ forgetful, $F(M)=\Gamma \otimes M$.

A graded algebra. Let $\Lambda$ be a graded $K$-algebra. Take

$\mathbf{A}=$ left $\Lambda$-modules, $\mathbf{M}=$ graded $K$-modules, $G$ and $F$ as in (2).

In particular, $\Lambda$ may be the Steenrod algebra over $Z_{p}$ ( $p$ prime).

A DG-algebra. Let $U$ be a DG(K-Mod)-algebra. Take

$$
\mathbf{A}=\text { left } U \text {-modules, } \mathbf{M}=\mathrm{DG}(K \text {-Mod), } G \text { and } F \text { as in (2). }
$$

Let $\mathrm{R}$ be any resolvent pair. Call a morphism $\sigma: B \rightarrow C$ a proper epic of $\mathbf{A}$ if $G(\sigma)$ has a right inverse in $\mathbf{M}$; since $G$ is faithful, it then follows that $\sigma$ is epic in A. Similarly, call a morphism $\kappa: A \rightarrow B$ proper monic in $\mathbf{A}$ if $G(\kappa)$ has a left inverse in $\mathbf{M}$. A short exact sequence $A \leadsto{ }^{\star} B \longrightarrow{ }^{\sigma} C$ is called proper if $\sigma$ is a proper epic or, equivalently, $\kappa$ is a proper monic; either statement implies that there is in $\mathbf{M}$ a biproduct diagram 


$$
0 \rightarrow G(A) \stackrel{G(\kappa)}{\stackrel{(}{\rightleftarrows}} G(B) \stackrel{G(\sigma)}{\rightleftarrows} G(C) \rightarrow 0 .
$$

Any morphism $\alpha$ of $\mathbf{A}$ is called proper if its factorization $\alpha=\lambda \tau$ (epic followed by monic) has $\tau$ proper epic and $\lambda$ proper monic. A long exact sequence is called proper in A if it factors, as in (11.3), into short exact sequences which are proper in the sense above. From (3) it follows that a DG-object $X$ for $\mathbf{A}$ is a proper long exact sequence if and only if $X$ has a contrasting homotopy.

The basic fact now is that those objects in $A$ which are the values $F(M)$ of the functor $F$ are "projective" objects for the proper epics, because of the following "lifting lemma":

LEMma 19.1. Given $\gamma: F(M) \rightarrow C$ and $\sigma: B \rightarrow C$ proper epic, the diagram

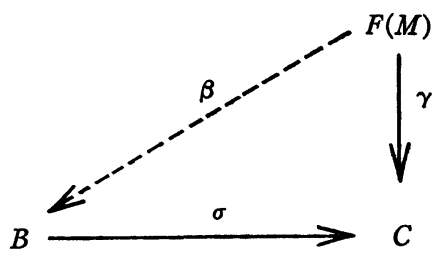

can be filled in at $\beta$ so as to be commutative.

In other words, each $\gamma$ with domain an $F(M)$ can be "lifted" through any proper epic $\sigma$.

PROOF. Since $\sigma$ is a proper, $G(\sigma)$ has a right inverse $t: G(C) \rightarrow G(B)$. By universal junction, the diagram

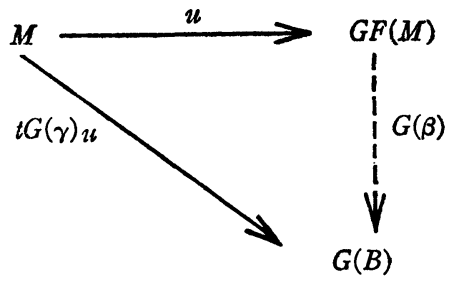

(in $\mathbf{M}$ )

can be filled in (uniquely) at $\beta$ so as to be commutative. Then $G(\sigma \beta) u=G(\sigma) t G(\gamma) u=G(\gamma) u$; since $G$ is faithful, $\sigma \beta=\gamma$, as required. This shows that the $\beta$ for (4) can be uniquely described in terms of $u$ and $t$.

Each object $A$ of A determines an object $F(G A)$ of the form $F(M)$; moreover, the theory of adjunction shows that $\mu_{A}: F G A \rightarrow A$ has 
$G\left(\mu_{A}\right) u_{A}=1: G A \rightarrow G A$; hence $G\left(\mu_{A}\right)$ has a right inverse, so is a proper epic. (In other language, there are "enough proper projectives" $F G(A)$ for all the objects $A$ of A.)

20. Resolutions. Continue with a resolvent pair $\mathbf{R}$. A resolution of $A$ is a DG-object

$$
0 \leftarrow A \stackrel{\epsilon}{\leftarrow} X_{0} \stackrel{d}{\leftarrow} X_{1} \stackrel{d}{\leftarrow} X_{2} \leftarrow \cdots
$$

over $A$ which is an exact sequence with $\epsilon$ and each $d$ a proper morphism. A resolution with a free basis $M$ is a resolution together with a graded object $M$ for $\mathbf{M}$ such that each $X_{n}$ is $F\left(M_{n}\right)$, as in the diagram

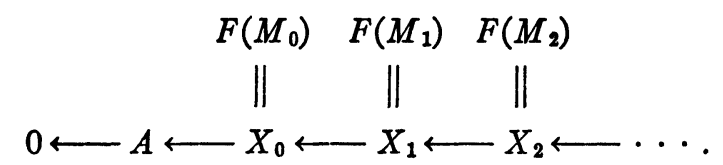

A resolution with a contraction $s$ is a resolution $X$ of $A$ together with a contracting homotopy $s$ for $G(X) \rightarrow G(A)$ such that $s^{2}=0$; that is, a diagram (1) in A together with a diagram

$$
0 \longleftarrow G(A) \underset{s_{-1}}{\stackrel{G(\epsilon)}{\leftrightarrows}} G\left(X_{0}\right) \underset{s_{0}}{\stackrel{G(d)}{\leftrightarrows}} G\left(X_{1}\right) \underset{s_{1}}{\stackrel{G(d)}{\leftrightarrows}} G\left(X_{2}\right) \underset{s_{2}}{\stackrel{G(d)}{\leftrightarrows}} \ldots
$$

in $M$ which satisfies (14.9) and $s^{2}=0$. As noted above, every resolution has such a contraction. The following result is basic for homological algebra:

Theorem 20.1 (Comparison Theorem). Let $\alpha: A \rightarrow B$ be $a$ morphism of $\mathbf{A}, \epsilon: X \rightarrow A$ a resolution of $A$ with a free basis and $\epsilon^{\prime}: Y \rightarrow B$ a resolution of $B$ with a contraction. Then there exists a morphism $\phi: X \rightarrow Y$ of $\mathrm{DG}(\mathrm{A})$ over $\alpha$ (i.e., with $\epsilon^{\prime} \phi_{0}=\phi_{0}$ ) and any two such $\phi$ are homotopic.

Proof. We are required to fill in the (commutative) diagram

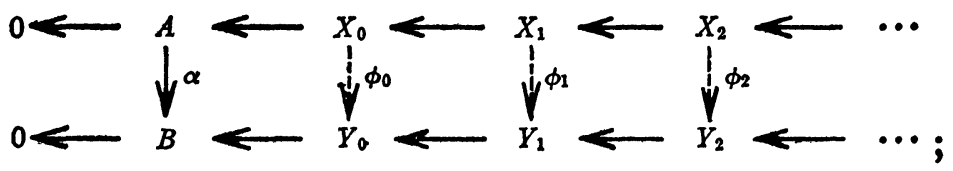

the bottom row is proper exact, so the lifting lemma will produce $\phi_{0}, \phi_{1}, \phi_{2}, \cdots$ in succession. The homotopy between two $\phi^{\prime} \mathrm{s}$ is obtained similarly. Moreover, the given universal junction $u_{n}: M_{n}$ 
$\rightarrow G F\left(M_{n}\right)=G\left(X_{n}\right)$ provides a unique or canonical choice for the $\phi$ 's, as follows.

ThEOREM 20.2 (CANONICAL COMPARISON). In the comparison theorem, let $X$ have the free basis $M$ and $Y$ the contraction $s$. Then there exists exactly one $\phi$ over $\alpha$ for which there are morphisms $w_{n}: M_{n}$ $\rightarrow G\left(Y_{n-1}\right)$ with

$$
G\left(\phi_{n}\right) u_{n}=s_{n-1} w_{n}: M_{n} \rightarrow G\left(Y_{n}\right), \quad n=0,1,2, \cdots .
$$

Indeed, $\phi_{n}$ is defined from (4) by recursion if we take

$$
w_{0}=G(\alpha \epsilon) u_{0}, \quad w_{n+1}=G\left(\phi_{n} d\right) u_{n+1}, \quad n=0,1,2, \cdots .
$$

The condition (4) may be stated more briefly as " $G(\phi) u$ factors through w."

Given two comparisons $\phi$ and $\phi^{\prime}$, there is a similar "canonical" homotopy between them.

There is also a canonical resolution, which may be described as a certain adjoint. Let $\mathrm{T}$ be the category with objects $(A, \epsilon: Y \rightarrow A, t)$; that is, objects $A$ of $\mathbf{A}$ plus resolutions $Y$ of $A$ with a specified contraction $t$, and with morphisms preserving all this structure (i.e., commuting with $\epsilon, d$, and $t$ ). Let $L: \mathbf{T} \rightarrow \mathbf{A}$ be the forgetful functor which assigns to each such object the underlying object $A$ of $\mathbf{A}$.

Theorem 20.3 (CANONICAL Resolution). The forgetful functor $L$ above has an adjoint $B: \mathrm{A} \rightarrow \mathrm{T}$. This adjoint assigns to each object $A$ a resolution $\epsilon: B(A) \rightarrow A$, called the bar resolution, which has both a free basis and a contraction $s$; moreover, each term $B_{n}(A)$ in this resolution has the form $B_{n}^{\prime} G A$ for some functor $B_{n}^{\prime}: \mathbf{M} \rightarrow \mathbf{A}$.

This gives a conceptual formulation of the result of the usual (painfully) explicit description of $B(A)$. That description (Homology, IX.7) does produce $B_{n} A$ in the stated form $B_{n}^{\prime} G A$ complete with free basis and contraction $s$ such that $s^{2}=0$. Now let $\left(A^{\prime}, \epsilon: Y \rightarrow A^{\prime}, t\right)$ be any resolution with a contraction $t$. From the explicit form of the contraction $s$ one may show that the canonical comparison $\phi: B(A)$ $\rightarrow Y$ over a morphism $\alpha: A \rightarrow A^{\prime}$ can be characterized as that (unique) map of DG-objects over $\alpha$ which commutes with the contracting homotopies; i.e., which satisfies

$$
G\left(\phi_{0}\right) s_{-1}=t_{-1} G(\alpha), \quad G\left(\phi_{n}\right) s_{n-1}=t_{n-1} G\left(\phi_{n-1}\right), \quad n=1,2, \cdots
$$

Thus $\phi$ is a map in our category $\mathbf{T}$, and is unique, so that the identity map $A \rightarrow L(A, \epsilon: B(A) \rightarrow A, s)$ is a universal junction, as required.

The bar resolution so described includes many familiar cases. In the multiplicative group case of $\$ 19$, it is exactly the complex first 
used by Eilenberg-MacLane to define the cohomology of groups; in the "algebra with bimodules" case it is exactly the complex first used by Hochschild to define the cohomology of an algebra.

21. Derived functors. Let $T: \mathrm{A} \rightarrow \mathrm{C}$ be an additive functor between abelian categories, where $\mathbf{A}$ comes from a resolvent pair $\mathbf{R}$. The bar resolution for $\mathbf{R}$ can be used to give a canonical definition of the derived functors $T_{n}: \mathbf{A} \rightarrow \mathbf{C}$ and their "connecting homomorphisms." By applying $T$ to each term $X_{n}$ of a DG-object for A we get a DG-object for $\mathbf{C}$, hence $T$ yields an additive functor $T_{*}: \mathrm{DG}(\mathbf{A}) \rightarrow \mathrm{DG}(\mathbf{C})$. In particular, $T_{*} B(A)$ is a DG-object. Now $B(A)$, as a resolution, has homology $H_{0} \cong A$ and $H_{n}=0$ for $n>0$, but, if the original functor $T$ is not exact, the complex $T_{*} B(A)$ need not have homology zero. Its homology defines the derived functors $T_{n}: \mathrm{A} \rightarrow \mathrm{C}$ as

$$
T_{n}(A)=H_{n}\left(T_{*} B(A)\right), \quad T_{n}(\alpha)=H_{n}\left(T_{*} B(\alpha)\right) .
$$

Next, if $E: A \mapsto D \longrightarrow C$ is a proper short exact sequence in $\mathbf{A}$, its propriety means that the corresponding short exact sequence $G(A) \longmapsto G(D) \longrightarrow G(C)$ in $\mathbf{M}$ is split. Hence, each functor $B_{n}^{\prime}$ of Theorem 20.3 carries this into a split exact sequence, so $B_{n}(A) \leadsto B_{n}(D) \longrightarrow B_{n}(C)$ is a split exact sequence in each dimension $n$, as is

$$
T_{*} B(E): 0 \rightarrow T_{*} B(A) \rightarrow T_{*} B(D) \rightarrow T_{*} B(C) \rightarrow 0 .
$$

This exact sequence of DG-objects for $\mathrm{C}$ has a connecting morphism (\$14) dropping dimensions by 1 . In view of the definition (1), this connecting morphism is a map

$$
E_{*}: T_{n}(C) \rightarrow T_{n-1}(A), \quad n=1,2, \cdots .
$$

Here each side may be read as a functor of the exact sequence $E$; it follows readily that $E_{*}$ is a natural transformation of these functors. All told, the "derived system" $\left\{T_{*}, E_{*}\right\}$ for $T$ consists of the functors $\left\{T_{0}, T_{1}, T_{2}, \cdots\right\}$ together with the natural connecting morphisms (2) for each proper $E$. Such a system is called a proper connected sequence of functors.

THEOREM 21.1. If $T: \mathbf{A} \rightarrow \mathbf{C}$ is an additive functor between abelian categories, while $\mathbf{R}$ is a resolvent pair for $\mathbf{A}$, then the derived functors for $T$ constitute an $\mathbf{R}$-proper connected sequence of functors with the following three properties:

(i) For $n>0$ and $M$ in $\mathbf{M}, T_{n}(F M)=0$;

(ii) For each proper short exact sequence $E: A \leftrightarrow \longrightarrow D \longrightarrow C$, the long sequence 
(3) $\cdots \longrightarrow T_{n}(A) \longrightarrow T_{n}(D) \longrightarrow T_{n}(C) \stackrel{E_{*}}{\longrightarrow} T_{n-1}(A) \longrightarrow \cdots$

of derived functors (with $T_{-1}=0$ ) is exact in $\mathbf{C}$;

(iii) If $T$ is right exact, then $T_{0}$ may be identified with $T$.

Moreover, for $T$ right exact, these three properties characterize the sequence of derived functors up to natural isomorphism.

Proof. The comparison theorem allows us to replace the bar resolution of $A$ by any other resolution $X \rightarrow A$ with a free basis and to compute $T_{n}(A)$ as $H_{n}\left(T_{*}(X)\right)$; this is usually the most efficient method of computation. The connecting morphisms can be calculated similarly from suitably constructed short exact sequences of resolutions. In particular, $A=F M$ has a free resolution $X$ with $X_{0}=F M$ and $X_{n}=0$ for $n>0$. As this resolution (and its homology) vanishes in properly positive dimensions, $T_{n}(F M)=0$ for $n>0$. This gives (i). The exact triangle (14.3) for the connecting morphisms yields the exactness of (3). Finally, when $T$ is right exact, it turns the right exact sequence $B_{1}(A) \rightarrow B_{0}(A) \rightarrow A \rightarrow 0$ into a sequence

$$
T B_{1}(A) \rightarrow T B_{0}(A) \rightarrow T A \rightarrow 0
$$

right exact in $\mathbf{C}$, hence shows $H_{0}\left(T_{*} B(A)\right) \cong T A$. Standard methods show that these three properties characterize the $T_{n}$; this is the "axiomatic description" of derived functors, familiar from special cases.

There is another characterization, by "co-universality." Let $\left\{S_{\sharp}, E_{*}\right\}$ be another R-proper connected sequence of functors; that is, a family of additive functors $\left\{S_{n}: \mathbf{A} \rightarrow \mathbf{C} \mid n=0,1,2, \cdots\right\}$ together with a function which assigns to each $n$ and each proper $E$ a morphism $E_{\sharp}: S_{n}(C) \rightarrow S_{n-1}(A)$ which is a natural transformation of functors of $E$. From the previous three properties one proves

THEOREM 21.2. If $T: \mathrm{A} \rightarrow \mathrm{C}$ is right exact with derived sequence $\left\{T_{*}, E_{*}\right\}$, while $\left\{S_{*}, E_{*}\right\}$ is any $\mathbf{R}$-proper connected sequence, then each naturalf: $S_{0} \rightarrow$ T extends uniquely to a morphism $f_{*}:\left\{S_{\sharp}, E_{*}\right\} \rightarrow\left\{T_{*}, E_{*}\right\}$.

Here $f_{*}$ is a morphism of proper connected sequences; that is, a family $\left\{f_{n}: S_{n} \rightarrow T_{n} \mid n=0,1, \cdots\right\}$ of natural transformations which commute with the connecting homomorphisms $E_{*}$ and $E_{*}$. Now let $\mathbf{C s q}(\mathbf{A}, \mathbf{C})$ denote the category of proper connected sequences of functors, with these morphisms, while $\mathbf{C}^{\mathbf{A}}$ denotes the functor category of additive functors. Compare these two categories by

$$
\mathrm{C}^{\mathrm{A}} \underset{I}{\stackrel{D}{\rightleftarrows}} \mathrm{Csq}(\mathrm{A}, \mathrm{C})
$$


here $D$ is the functor which assigns to each additive $T$ its derived sequence, while $I$ takes the initial term of any connected sequence. In this language, the theorem above states that if $T$ is right exact there is a (natural) bijection

$$
\operatorname{Nat}\left(I\left(S_{*}\right), T\right) \cong \mathrm{Csq}\left(S_{*}, D T\right) .
$$

In other words, the functor of $S_{*}$ on the left is corepresentable, in the category $\mathrm{Csq}$, by the object $D T$. As with any corepresentation, this property characterizes the derived sequence $D T$. More generally, let $T: \mathbf{A} \rightarrow \mathbf{C}$ be any additive functor and $L T$ a proper connected sequence with initial term $(L T)_{0}=T$. Restricting a morphism of connected sequences to the initial term gives a map

$$
\operatorname{Csq}\left(S_{*} L T\right) \rightarrow \operatorname{Nat}\left(I\left(S_{*}\right), T\right) \text {. }
$$

When this map is a bijection, $L T$ is called the sequence of left satellites of $T$. Thus, for a right exact $T$, the derived functors agree with the satellites.

The exact triangle of (14.3) yields another exactness result for derived functors.

Theorem 21.3. Let $\mathbf{R}$ be a resolvent pair of categories, and $S: T^{\prime} \rightarrow T$ $\rightarrow T^{\prime}$ a sequence of natural transformations of additive functors from $\mathbf{A}$ to the abelian category $\mathbf{C}$ such that each object $M$ yields a short exact sequence $T^{\prime}(F M) \longmapsto T(F M) \longrightarrow T^{\prime}(F M)$ in C. Then $S$ determines a family of transformations $S_{*}: T_{n}^{\prime}(A) \rightarrow T_{n-1}^{\prime}(A)$ for $n=1,2, \cdots$, natural in $A$, and the long sequence

$$
\cdots \rightarrow T_{n}^{\prime}(A) \rightarrow T_{n}(A) \rightarrow T_{n}^{\prime \prime}(A) \stackrel{B_{*}}{\longrightarrow} T_{n-1}^{\prime}(A) \rightarrow \cdots
$$

is exact in $\mathbf{C}$ for every object $A$ of $\mathbf{A}$.

Proof. Since each object $B_{n}(A)$ has the form $F\left(M_{n}\right)$ for some $M_{n} \in \mathbf{M}$, the sequence $T_{*}^{\prime} B(A) \longrightarrow T_{*} B(A) \longrightarrow T_{*}^{\prime \prime} B(A)$ is a short exact sequence of DG-objects for $\mathrm{C}$; its connecting morphism gives the desired $S_{*}$.

These methods apply also to a contravariant functor $T: \mathbf{A}^{\mathbf{o p}} \rightarrow \mathbf{C}$. Since $T$ reverses the order of composition in $\mathbf{A}$, the corresponding functor $T^{*}$ turns any DG-object $X$ for $\mathbf{A}$ into a "cochain complex":

$$
T^{*}(X): T\left(X_{0}\right) \rightarrow T\left(X_{1}\right) \rightarrow T\left(X_{2}\right) \rightarrow \cdots, T(d)=\delta ;
$$

that is, a graded object for $\mathbf{C}$ with $\delta$ of degree +1 and $\delta \delta=0$. The usual construction $(\operatorname{ker} \delta) /\left(\operatorname{im} \delta\right.$ ) yields the cohomology $H^{n}\left(T^{*}(X)\right)$, normally written with an upper index $n$. The derived functors and connecting morphisms are 


$$
T^{n}(X)=H^{n}\left(T^{*} B(A)\right), \quad E^{*}: T^{n}(A) \rightarrow T^{n+1}(C),
$$

and each proper short exact sequence $E: A \gg D \longrightarrow C$ in $\mathrm{A}$ yields a long exact sequence

$$
\cdots \rightarrow T^{n}(C) \rightarrow T^{n}(D) \rightarrow T^{n}(A) \stackrel{E^{*}}{\rightarrow} T^{n+1}(C) \rightarrow \cdots \text { in C. }
$$

If $T$ is left exact (i.e., carries right exact sequences in $\mathbf{A}$ to left exact sequences in $\mathbf{C}$ ), then $T^{0}=T$. The analogue of (4) holds.

This treatment of derived functors subsumes many cases, corresponding to the instances of resolvent pairs listed in $\$ 19$.

The relative torsion product (Hochschild). Consider the resolvent pair given by a pair $S \subset R$ of rings. A proper exact sequence of left $R$-modules is then an exact sequence of $R$-modules which splits as a sequence of $S$-modules. For a fixed right $R$-module $D$, let $T_{D}: \mathrm{A} \rightarrow \mathrm{Ab}$ be the additive functor from left $R$-modules to abelian groups defined by $T_{D}(A)=D \otimes_{R} A$. Since a ring $R$ is just a $Z$-algebra, this functor is right exact, by $\$ 18$. The derived functors of $T_{D}$ are known as the relative torsion products and are written as $\operatorname{Tor}_{n}^{(R, S)}(D, A)$; $\operatorname{Tor}_{0}$ is just $D \otimes_{R} A=T_{D}(A)$. Theorem 21.1 yields a connecting homomorphism and a corresponding long exact sequence in the second argument $A$. To get a corresponding result for the first argument $D$ we may use Theorem 21.3. Since $F(M)=R \otimes_{S} M$,

$T_{D}(F M)=D \otimes_{R}\left(R \otimes_{S} M\right)=\left(D \otimes_{R} R\right) \otimes_{S} M \cong D \otimes_{S} M, \quad M \in \mathbf{M}$.

Thus, if $L: D^{\prime} \gg D \rightarrow D^{\prime \prime}$ is an $S$-split exact sequence of right $R$ modules, the corresponding sequence $T_{D^{\prime}}(F M) \rightarrow T_{D}(F M) \rightarrow T_{D^{\prime \prime}}(F M)$ is

$$
0 \rightarrow D^{\prime} \otimes_{S} M \rightarrow D \otimes_{S} M \rightarrow D^{\prime \prime} \otimes_{S} M \rightarrow 0,
$$

and is exact because $L$ is $S$-split. Hence Theorem 21.3 yields connecting homomorphisms $L_{*}$ : $\operatorname{Tor}_{n}^{\left(R_{2} S\right)}\left(D^{\prime \prime}, A\right) \rightarrow \operatorname{Tor}_{n-1}^{(R, S)}\left(D^{\prime}, A\right)$ and a corresponding long exact sequence in the first argument $D$ of the relative torsion product.

The relative Ext. Let $\mathbf{R}$ be any resolvent pair. Each $D$ in $\mathrm{A}$ gives a functor $T_{D}(A)=\operatorname{hom}_{\mathrm{A}}(A, D)$ which is contravariant; that is, a functor $T_{D}: A^{\mathrm{OP}} \rightarrow \mathrm{Ab}$. It is left exact. Its derived functors, found as in the contravariant case above, are the relative ext functors $\operatorname{ext}_{\mathbf{A},{ }_{M}}(A, D)$. An element of ext $^{n}$ may be regarded as a congruence class of proper long exact sequences from $D$ to $A$ with $n$ intermediate terms; the congruence relation is that introduced by Yoneda ( $\mathrm{Ho}$ mology, III.5 and XII.5). The theorems above yield connecting homomorphisms and the usual long exact sequences in $A$ or in $D$. 
The cohomology of groups. Let $G$ be a multiplicative group and $\mathbf{R}$ the corresponding resolvent pair, as in $\$ 19$. The additive group $Z$ of integers is trivially a $G$-module (i.e., in $\mathbf{A}$ ). The functors ext $\mathrm{t}_{\mathbf{A}, \mathbf{M}}^{n}(Z, A)$ $=H^{n}(G, A)$ are the cohomology groups of the group $G$ with coefficients in the $G$-module $A$. The homology groups of $G$ are similarly defined (Homology, X.5).

The cohomology of algebras. Let $\Gamma$ be a $K$-algebra and $\mathbf{R}$ the corresponding resolvent pair, with $\mathbf{A}$ consisting of $\Gamma$-bimodules, $\mathbf{M}$ of right $\Gamma$-modules. The algebra $\Gamma$ itself is trivially a $\Gamma$-bimodule, with left and right operation given by the product in $\Gamma$. Let $A$ be a $\Gamma$ bimodule. The functor $\operatorname{ext}_{\mathbf{A}, \mathbf{M}}^{n}(\Gamma, A)$ is the Hochschild cohomology group $H^{n}(\Gamma, A)$ of the algebra $\Gamma$ with coefficients in $A$. In this case, each $\operatorname{hom}_{\mathbf{A}}(B, A)$ has the structure of a $K$-module, so ext $t_{\mathbf{A}, \mathbf{M}}^{n}$ and $H^{n}$ can be regarded as functors to the category $K$-Mod.

All these cases use projective resolutions, such as the bar resolution. There is a dual case of covariant functors $T: \mathbf{A} \rightarrow \mathbf{C}$ which are left exact; the most important case is that in which $\mathbf{A}$ is the category of sheaves $S$ on some topological space $X$, and $T(S)=\Gamma(S)$, the group of global cross sections of the sheaf $S$, is left exact. The derived functors of $\Gamma$ are the cohomology groups of $X$ with coefficients in the sheaf $S$. In this way the notion of derived functor (or of satellite functor) unifies the ideas of cohomology of groups or of spaces. These derived functors may be computed from injective resolutions, in particular from a canonical "flabby" resolution introduced in [39] by Godement. This resolution has been extended to more general categories by Huber [52]; Schafer has shown in [90] that it is the exact dual of the categorical bar resolution.

Here we have spoken of sheaves of abelian groups (or of modules over some fixed ring). It is natural to consider, more generally, sheaves with values in a suitable abelian category; this has been effectively carried out by Gray [40]. The category must be one in which the direct limit functor is exact; derived functors of this functor have been studied [83], [88].

22. Differential graded algebras. Fix a commutative ground ring $K$, and regard it as a differential graded algebra (with trivial differential and grading). An augmented differential graded $K$-algebra $U$ (a DGA-algebra for short) is a DG(K-Mod)-algebra $U$ together with a morphism $\epsilon=\epsilon_{U}: U \rightarrow K$ of such algebras, called the augmentation. By neglect, each such algebra $U$ can be regarded simply as a graded $K$-algebra $\operatorname{Gr}(U)$; here $\mathrm{Gr}$ is a forgetful functor

$$
\text { Gr: } \operatorname{Alg}(\mathrm{DG}(K-\mathbf{M o d})) \rightarrow \operatorname{Alg}(\mathrm{G}(K-\mathbf{M} \text { od })) .
$$


(Forget the differential, but remember the grading.)

The various categories of modules at hand (for notation, see Proposition 18.1) are:

$\mathrm{G}(K$-Mod $)$, the graded $K$-modules $M$; call $M$ connected if $M_{0}=K$;

$\mathrm{DG}(K$-Mod), the differential graded $K$-modules (DG- $K$-modules for short);

(Gr $U)$-Mod(G(K-Mod)), the left modules over the graded $K$ algebra $\operatorname{Gr} U$; by neglect, each such module is also a graded $K$ module;

$U$-Mod(DG(K-Mod)), the left $U$-modules $X$. Each such is automatically a DG- $K$-module, and, by further neglect, a G- $K$-module $\operatorname{Gr}(X)$. Also, $K$ itself is a left $U$-module ${ }_{\epsilon} K$, when the left operation of $u$ on $k \in K$ is defined by pull-back along the augmentation $\epsilon_{U}$ as $u k$ $=\epsilon(u) k$. A left $U$-module $X$ is augmented if there is given a map $\epsilon=\epsilon_{X}: X \rightarrow_{\epsilon} K$ of left $U$-modules.

Each $U$-module is a DG-object and so is like a resolution; in particular, there are comparison theorems for $U$-modules which depend on the following definitions of "free" and "contractible" such modules.

A (connected) free basis $M$ for an augmented left $U$-module $X$ is a connected graded submodule $M \subset \mathrm{Gr} X$ with the same augmentation $\left(\epsilon_{X} k=k\right.$ for all $\left.k \in M_{0}=K\right)$ such that the correspondence $u \otimes m \rightarrow u m$ provides an isomorphism $\theta: U \otimes M \cong X$ of augmented graded $K$ modules - and hence automatically an isomorphism of $\operatorname{Gr}(U)$-modules. (However, $\theta$ is not required to be an isomorphism of $U$-modules; that is, $\theta$ need not commute with $d$.) Given a free basis $M$, the injection $t_{-1}: K \rightarrow K \subset M_{0}$ is then a map $t_{-1}: K \rightarrow X$ of $\mathrm{G}(K$-Mod) with $\epsilon t_{-1}=1$.

A contraction $s$ for an augmented left $U$-module $Y$ consists of maps

$$
s_{-1}: K \rightarrow Y_{0}, \quad s_{n}: Y_{n} \rightarrow Y_{n+1}, \quad n=0,1,2, \cdots,
$$

of $K$-modules such that

(2) $\epsilon_{Y} s_{-1}=1_{K}, \quad d s_{0}+s_{-1} \epsilon=1_{Y_{0}}, \quad d s_{n+1}+s_{n} d=1_{Y_{0}}, \quad s_{n} s_{n-1}=0$.

The same formulas, specialized by setting $U=K$, define a contraction for an augmented DG- $K$-module. An argument like that for the canonical comparison of $\$ 20$ now proves

Theorem 22.1 (CANonical DG-Comparison). If $U$ is a DGAalgebra, $X$ an augmented $U$-module with a free basis $M$, and $Y$ an augmented $U$-module with a contraction $s$, there is a unique map $\phi: X \rightarrow Y$ of augmented $U$-modules such that

$$
\phi\left(M_{0}\right) \subset s_{-1} K, \quad \phi\left(M_{n}\right) \subset s_{n-1} Y_{n-1}, \quad n=1,2, \cdots .
$$


These conditions amount to the statement that $\phi$ on $M_{n}$ "factors through $s . "$

As in the comparison Theorem 20.1, two comparisons $\phi$ and $\phi^{\prime}$ (not necessarily canonical) are homotopic-via a canonical homotopy; two homotopies between the same comparison are themselves homotopic, and so on through "higher homotopies." To formulate this, after the manner of Dold's treatment [12] of the Eilenberg-Zilber theorem for simplicial complexes, introduce to each pair of augmented $U$-modules $X$ and $Y$ the DG- $K$-module $\operatorname{Hom}_{U}(X, Y)$ with terms $\mathrm{Hom}_{n}$ given by

$$
\begin{aligned}
& \operatorname{Hom}_{0}(X, Y)=\{\text { maps } f: X \rightarrow Y \text { of } U \text {-modules (of degree } 0 \text { ) }\}, \\
& \operatorname{Hom}_{n}(X, Y)=\{\text { maps } g: X \rightarrow Y \text { of } \operatorname{Gr}(U \text { )-modules (of degree } n \text { ) }\},
\end{aligned}
$$

and with differential $D: \mathrm{Hom}_{n} \rightarrow \mathrm{Hom}_{n-1}$ defined exactly as in (14.5) - the special convention for $\mathrm{Hom}_{0}$ insures that this yields a graded module and not a $Z$-graded module, as was the case in $\$ 14$. If $X$ has a free basis with the corresponding $t_{-1}: K \rightarrow X$, we augment $\operatorname{Hom}_{U}(X, Y)$ by $\epsilon=\epsilon_{H}$ defined from $t_{-1}$ as

$$
\text { (4) } \epsilon_{H}: \operatorname{Hom}_{0}(X, Y) \rightarrow K, \quad \epsilon_{H} f=\epsilon_{Y} f_{0} t_{-1} 1 \quad(1 \text { the identity of } K) \text {. }
$$

TheOREM 22.2 (DG-Comparison homotopy). If $U$ is a DGAalgebra, $X$ an augmented $U$-module with a (connected) free basis $M$, and $Y$ an augmented $U$-module with a contraction s, then the $D G$-K-module $\operatorname{Hom}_{U}(X, Y)$ has a contraction $S$.

Proof. We shall describe a "canonical" contraction. To give $S_{-1}: K \rightarrow$ Hom $_{0}$, it suffices to define $S_{-1}$ on the identity 1 of $K$ to be some element of $\operatorname{Hom}_{0}(X, Y)$; we take $S_{-1}(1)$ to be the canonical comparison $\phi: X \rightarrow Y$ constructed in Theorem 22.1. For $n \geqq 0, S_{n}: \mathrm{Hom}_{n}$ $\rightarrow$ Hom $_{n+1}$ assigns to each $\operatorname{Gr}(U)$-module map $g: X \rightarrow Y$ another such map $S_{n} g$. But $X \cong U \otimes M$ as a $\operatorname{Gr}(U)$-module, so $S_{n} g$ is determined by its values on $M$; we give these by recursion on the degree $t$ in $M$ as

$$
\left(S_{n} g\right) m=s\left[g+(-1)^{n+1}\left(S_{n} g\right) d\right] m, \quad m \in M_{t} .
$$

One then verifies that $S$ is a contraction (of square zero).

"Factoring out" by the action of $U$ will turn each $U$-module into its "reduced" DG-module. In detail, let $J$ be the kernel of the augmentation $\epsilon_{U}: U \rightarrow K$, so that $J_{\curvearrowright} \rightarrow U \longrightarrow K$ is an exact sequence of graded $K$-modules. Regard $K$ as a right $U$-module $K_{e}$, by "pullback" along $\epsilon_{U}$. For each left $U$-module $A$, the tensor product $K \otimes_{U} A$ is then a DG- $K$-module called the reduced module $\bar{A}$ of $A$. Since the tensor product is right exact, there is an isomorphism

$$
A=K \cdot \otimes_{U} A \cong A / J A
$$


(Homology, Proposition X.10.1), where $J A$ is the DG- $K$-module consisting of all sums of products $x a$ for $x \in J, a \in A$. The corresponding projection $f: A \rightarrow A / J A \cong \bar{A}$ is a map of DG-K-modules, and is natural. If $A$ is augmented, so is $\bar{A}$. If $A$ has a free basis $M,(5)$ and $\theta: U \otimes M$ $\cong A$ gives an isomorphism $\operatorname{Gr}(\bar{A}) \cong M$.

23. The bar construction. For DGA-algebras the bar resolution takes a new form.

THEOREM 23.1. If $U$ is a DGA-algebra over $K$, there is an augmented left $U$-module $B(U)$ which has a (connected) free basis $M$ and also a contraction $s$ and is such that $M$ is exactly the image of $s$. This property characterizes $B(U)$ up to isomorphism.

Proof. As in $\$ 19, U$ gives a resolvent pair. The corresponding bar resolution assigns to each left $U$-module $A$ a DG-object (for the category of $U$-modules) which we write as $B(U, A)$ to exhibit the dependence upon $U$; in particular, we write $B(U)$ for $B\left(U,{ }_{\epsilon} K\right)$. Now $A$ is in the category $U-\operatorname{Mod}(\mathrm{DG}(K-\mathrm{Mod}))$, so

$$
B(U, A), B(U) \in \mathrm{DG}(U-\operatorname{Mod}(\mathrm{DG}(K-\operatorname{Mod}))) .
$$

This expression exhibits $B(U)$ as a "complex of complexes" (a DG-object for a category of DG-objects), with two gradings and two differentials. By taking a new grading which is the sum of these two and a new differential which is a suitably signed sum of these twoas discussed in Homology, X.9-we turn $B(U)$ into a singly graded object $B^{\bullet}(U)$ with a single differential which satisfies the conditions of this theorem. For example, the fact that $M$ is the image of $\left(_{-1} K\right.$ $\left.=M_{0}, s_{n}\left(B^{\bullet}(U)\right)_{n}=M_{n+1}\right)$ follows from the corresponding property of the original bar resolution. The uniqueness of $B^{\circ}(U)$ is an immediate consequence of the canonical DG-comparison, because by (22.3) the canonical comparison of $B^{\circ}(U)$ to itself must be the identity.

This augmented left $U$-module $B^{\circ}(U)$ is Cartan's (acyclic) bar construction. Its corresponding DG-K-module $\bar{B}(U)$ is the (reduced) bar construction due to Eilenberg-MacLane. Both may be described by explicit formulas (Homology, X.10). The corresponding projection $B^{\bullet}(U) \rightarrow \bar{B}^{\bullet}(U)$ is the algebraic analogue of a principal fibre bundle with fibre $U$, so that $\bar{B}^{\bullet}(U)$ is a "classifying space" for $U$. If $\mu: U \rightarrow U^{\prime}$ is a map of DGA-algebras (over the same $K$ ), then the $U^{\prime}$-module $B^{\bullet}\left(U^{\prime}\right)$ may be regarded, by pull-back along $\mu$, as a $U$-module. Since $B^{\circ}\left(U^{\prime}\right)$ has a contraction, the DG-comparison gives a canonical map $B^{\bullet}(\mu): B^{\bullet}(U) \rightarrow B^{\bullet}\left(U^{\prime}\right)$ and a corresponding map for the reduced modules. Thereby $\bar{B}^{\bullet}$ becomes a functor on DGA-algebras to DG- $K$ modules. 
Now suppose that the algebra $U$ is commutative. This implies that the product map $p: U \otimes U \rightarrow U$ for $U$ is a map of DGA-algebras. Hence $B^{*}(U)$ may be regarded, by pull-back along $p$, as a left $(U \otimes U)$-module with a contraction. On the other hand $B^{*}(U) \otimes B^{\bullet}(U)$ is a left $(U \otimes U)$-module with $M \otimes M$ as free basis. The DG-comparison thus yields a canonical map $B^{\bullet}(p): B^{\bullet}(U) \otimes B^{\bullet}(U) \rightarrow B^{\bullet}(U)$. Using the "canonical" characterization of $B^{*}(p)$, it follows that $B^{\circ}(U)$ is a DGA-algebra under this product. This product factors through the projection $B^{*} \rightarrow \bar{B}^{\bullet}$, so $\bar{B}^{\bullet}(U)$ is also a DGA-algebra. Thus for a commutative $U$ the reduced bar construction may be iterated to give DGA-algebras $\left(\bar{B}^{*}\right)^{n}(U), n=1,2, \cdots$.

The bar construction was devised to treat topological spaces of type $K(\Pi, n)$-spaces which have only one (abelian) homotopy group II in dimension $n$. The (minimal simplicial) chain complex of such a space is a DGA-algebra over $Z$. A theorem of Eilenberg-MacLane asserts that this chain complex is equivalent to $\left(\bar{B}^{*}\right)^{n}(Z(\Pi))$, where $Z(\Pi)$ is the integral group ring of $\Pi$.

For $\Pi$ abelian, $Z(\Pi)$ is a hopf algebra with a commutative product (and trivial grading and differential). In general, let $U$ be a DG(K-Mod)-hopf algebra ( $\$ 17)$; via the co-unit as augmentation, $U$ is then a DGA-algebra. Using the DG-comparison, one then shows that $B^{*}(U)$ and $\bar{B}^{\bullet}(U)$ are also DG(K-Mod)-hopf algebras; for example, the commutativity condition (17.4) for a hopf algebra is verified by showing that each composite map is canonical, using the standard contraction in each tensor product. By iteration, the $\left(\bar{B}^{\circ}\right)^{n}(U)$ are also $\mathrm{DG}(K$-Mod)-hopf algebras.

\section{Chapter V. Higher Homotopies}

24. Proper categories. In the bar construction and its iterates, the natural coproduct is not commutative, but only commutative "up to higher homotopies." The same is true of the diagonal map for the chain complex of a simplicial set. Current investigation of these higher homotopies, carried out in collaboration with J. F. Adams, indicates that there are so many of them that general conceptual methods are needed for their treatment. In this chapter we describe two special types of categories which promise to be useful in this treatment.

All the special categories $\mathbf{H}$ to be considered have as objects the natural numbers $\{0,1,2, \cdots\}$; we write

$$
\mathrm{H}\left(\begin{array}{l}
m \\
n
\end{array}\right)
$$


for $\operatorname{hom}_{\mathrm{H}}(m, n)$. A product and permutation category $\mathrm{H}$ (a PROP, for short) is a category with the natural numbers as objects and with two further structures, as follows:

(i) For each $n$, the symmetric group $S(n)$ on $n$ letters is given as a subgroup of the group of all invertible elements of

$$
\mathrm{H}\left(\begin{array}{l}
n \\
n
\end{array}\right)
$$

in particular, the identity permutation in $S(n)$ is the identity morphism $1_{n}: n \rightarrow n$.

(ii) There is given a functor $\otimes: \mathbf{H} \times \mathbf{H} \rightarrow \mathbf{H}$, called the product and written between its arguments, and with object function $m \otimes m^{\prime}$ $=m+m^{\prime}$. Its mapping function therefore assigns to morphisms $f: m \rightarrow n$ and $f^{\prime}: m^{\prime} \rightarrow n^{\prime}$ of $\mathrm{H}$ a morphism $f \otimes f^{\prime}: m+m^{\prime} \rightarrow n+n^{\prime}$. The standard requirement that $\otimes$ preserves the composition reads

$$
(f \circ g) \otimes\left(f^{\prime} \circ g^{\prime}\right)=\left(f \otimes f^{\prime}\right) \circ\left(g \otimes g^{\prime}\right),
$$

valid whenever the composites on the left side are defined.

There are three axioms. First, we require that the mapping function $\otimes$ be associative; that is, that

$$
\left(f \otimes f^{\prime}\right) \otimes f^{\prime \prime}=f \otimes\left(f^{\prime} \otimes f^{\prime \prime}\right) .
$$

Second, for $\sigma \in S(n)$ and $\sigma^{\prime} \in S\left(n^{\prime}\right)$, let $\sigma \times \sigma^{\prime} \in S\left(n+n^{\prime}\right)$ denote that permutation of $n+n^{\prime}$ letters which acts on the first $n$ letters as does $\sigma$ and on the remaining $n^{\prime}$ letters as does $\sigma^{\prime}$. We require that

(3) $\sigma \otimes \sigma^{\prime}=\sigma \times \sigma^{\prime}: n+n^{\prime} \rightarrow n+n^{\prime}, \quad \sigma \in S(n), \sigma^{\prime} \in S\left(n^{\prime}\right)$.

Third, let $\tau$ denote the nonidentity element (the transposition) in $S(2)$. For any natural numbers $m$ and $m^{\prime}$, let $\tau_{\left(m, m^{\prime}\right)}$ be that permutation in $S\left(m+m^{\prime}\right)$ which interchanges the first block of $m$ letters and the second block of $m^{\prime}$ letters. For any $f: m \rightarrow n$ and $f^{\prime}: m^{\prime} \rightarrow n^{\prime}$ in $\mathbf{H}$, we require that

$$
\tau_{\left(n, n^{\prime}\right)}\left(f \otimes f^{\prime}\right)=\left(f^{\prime} \otimes f\right) \tau_{\left(m, m^{\prime}\right)} .
$$

For example, regard each natural number $n$ as a finite ordinal; that is, as the set $n=\{0,1, \cdots, n-1\}$ of all the preceding ordinals. Let $\mathbf{N}$ denote the category with objects all finite ordinals and morphisms $f: m \rightarrow n$ all functions on the set $m$ to the set $n$-plus, for good measure, the identity morphism 1: $0 \rightarrow 0$. In this category there is an evident coproduct diagram $m \rightarrow m+n \leftarrow n$. Take this coproduct as the functor $\otimes$, and take the one-one functions $n \rightarrow n$ as the permutations. Then $\mathbf{N}$ is a PROP. 
Again, let $\mathbf{C}$ be any category with finite products and a terminal object $T$. For a fixed object $C$, write $C^{0}=T$ and $C^{n}=C \times \cdots \times C$ ( $n$ factors). Take $\mathrm{H}_{C}$ to be the subcategory of $\mathrm{C}$ with objects all powers $C^{n}$ (identify them with the objects $n$ ) and with morphisms

$$
\mathrm{H}_{C}\left(\begin{array}{l}
m \\
n
\end{array}\right)=\operatorname{hom}_{\mathrm{C}}\left(C^{m}, C^{n}\right) \text {. }
$$

This subcategory has $C^{m} \times C^{n}=C^{m+n}$ as a product; take this product to be the functor $\otimes$. For $\sigma \in S(n)$, take $\sigma^{*}: C^{n} \rightarrow C^{n}$ to be the corresponding morphism of $\mathbf{C}$; that is, the morphism characterized in terms of the $n$ projections $p_{j}: C^{n} \rightarrow C$ by $p_{\sigma j} \sigma^{*}=p_{j}$. With these structures, $\mathbf{H}_{C}$ is a PROP. An arbitrary PROP $\mathbf{H}$ is said to act on the object $C$ of $\mathbf{C}$ if there is given a morphism $\mathbf{H} \rightarrow \mathrm{H}_{C}$ of PROPs.

Another useful construction yields the free PROP on a given set of generators and also the PROP with given generators and relations. With some formal complications, one may give existence proofs for these objects and obtain for them the expected properties of free objects.

These categories are adapted to the study of universal algebra. For instance, consider monoids; that is, sets $C$ with two maps $p: C \times C$ $\rightarrow C$ and $u: T \rightarrow C$ (with $T$ the one-point set) satisfying the usual conditions

$p(p \times 1)=p(1 \times p): C^{3} \rightarrow C, p(u \times 1)=1=p(1 \times u): C \rightarrow C$,

which state that $p$ is associative and $u$ a left and right identity. Introduce a corresponding PROP $\mathbf{M}$ ( $M$ for monoid) described as the free PROP on two generators $p: 2 \rightarrow 1$ and $u: 0 \rightarrow 1$ subject to the relations

$$
p(p \otimes 1)=p(1 \otimes p): 3 \rightarrow 1, \quad p(u \otimes 1)=1=p(1 \otimes u): 1 \rightarrow 1 .
$$

If $\mathbf{M}$ acts on a set $C$, then $C$ is a monoid, in the usual sense, and conversely. With analogous definitions, if $\mathbf{M}$ acts on a $K$-module $A$, then $A$ is a $K$-algebra. This is parallel to the proposal of Eckmann-Hilton to define "monoid" (and similarly "group") in an arbitrary category which has finite products.

In Lawvere's study [67] of universal algebra, an algebraic theory A is defined to be a category with objects the natural numbers such that each natural number $n$ is the categorical product of the object 1 with itself $n$ times. From the properties of products, it follows then that

$$
S(n) \subset \mathbf{A}\left(\begin{array}{l}
n \\
n
\end{array}\right)
$$


and hence that every algebraic theory is a PROP, when $\otimes$ is taken to be the categorical product (conversely, however, there are PROPs which are not algebraic theories). Each algebraic theory determines the category $\mathbf{E n s}^{(\mathbf{A})}$ of all algebras of type $\mathbf{A}$, where an algebra of type $A$ can be described as a product-preserving functor $\mathbf{A} \rightarrow$ Ens to the category of sets. Moreover, each object-preserving functor $F: \mathbf{A} \rightarrow \mathbf{B}$ between algebraic theories determines a functor $F^{*}: \mathbf{E n s}^{(B)} \rightarrow$ Ens $^{(A)}$ between categories of algebras; such an $F^{*}$ is termed an algebraic functor. Lawvere proves that every such algebraic functor has an adjoint. This result includes many familiar cases of adjoints; for example, if $F$ is the process of forming the bracket product $[a, b]=a b-b a$ from the usual product in associative algebras, then $F^{*}$ assigns to each associative algebra (over a field) the corresponding Lie algebra; its adjoint constructs to a Lie algebra the enveloping associative algebra. In this case, the Poincaré-Birkhoff-Witt theorem asserts that the corresponding universal junction is a monomorphism. A central problem is that of determining general conditions on $\mathbf{A}, \mathbf{B}$, and $F$ sufficient to insure that the appropriate universal junction is monic.

25. PACTs. We now consider the special categories appropriate to higher homotopies. These categories involve Permutations, $A$ ddition, Composition and Tensor product, so will be dubbed PACTs. Let $K$ be a fixed commutative ground ring, and let "complex" mean $\mathrm{DG}_{\mathbf{z}}$-object for $K$-Mod. A PACT $\mathbf{P}$ is a category with objects the natural numbers and with the following additional structures.

(i) Each

$$
\mathbf{P}\left(\begin{array}{c}
m \\
n
\end{array}\right)
$$

is a complex, with differential written as $D$ and with the degree of each morphism $f: m \rightarrow n$ as $|f|$. Composition in $\mathbf{P}$ is required to be a morphism

$$
\mathbf{P}\left(\begin{array}{l}
m \\
n
\end{array}\right) \otimes \mathbf{P}\left(\begin{array}{l}
k \\
m
\end{array}\right) \rightarrow \mathbf{P}\left(\begin{array}{l}
k \\
n
\end{array}\right)
$$

of complexes. Here the tensor product on the left has the standard DG structure ( $\$ 16)$. of

(ii) For each $n$, the symmetric group $S(n)$ is a given subgroup

$$
\mathrm{P}_{0}\left(\begin{array}{l}
n \\
n
\end{array}\right)
$$


(iii) There is a functor $\otimes: \mathrm{P} \times \mathrm{P} \rightarrow \mathrm{P}$, called tensor product, with object function $m \otimes n=m+n$ and mapping function a morphism

$$
\mathbf{P}\left(\begin{array}{c}
m \\
n
\end{array}\right) \otimes \mathbf{P}\left(\begin{array}{c}
m^{\prime} \\
n^{\prime}
\end{array}\right) \rightarrow \mathbf{P}\left(\begin{array}{c}
m+m^{\prime} \\
n+n^{\prime}
\end{array}\right)
$$

of complexes. Observe that the morphisms of $\mathbf{P}$ have degrees, so that the standard sign crops up in the definition of the composition in $\mathbf{P} \times \mathbf{P}$ : this amounts to inserting in (24.1) a sign $(-1)^{e}$ with $e=|f|\left|g^{\prime}\right|$.

There are three axioms, which are exactly the axioms (2)-(4) for PROPs, with a sign $(-1)^{|f|\left|f^{\prime}\right|}$ inserted in (4). Each PACT has homology $H(\mathbf{P})$, defined by

$$
(B \mathrm{P})\left(\begin{array}{c}
m \\
n
\end{array}\right)=B\left(\mathrm{P}\left(\begin{array}{c}
m \\
n
\end{array}\right)\right),
$$

with composition, permutation, and tensor product induced by the same structures from $\mathbf{P}$. Then $H(\mathbf{P})$ is also a PACT, with differential zero.

Each complex $X$ determines a PACT $\mathbf{X}$. Set $X^{0}=K$ and $X^{m}$ $=X \otimes \cdots \otimes X(m$ factors $)$ and take

$$
\mathbf{X}\left(\begin{array}{c}
m \\
n
\end{array}\right)=\operatorname{hom}\left(X^{m}, X^{n}\right)
$$

with differential $D$ of (14.4). The evident composition is known to be a morphism of complexes. If $f: X^{m} \rightarrow X^{n}$ and $f^{\prime}: X^{m^{\prime}} \rightarrow X^{n^{\prime}}$, the tensor product $f \otimes f^{\prime}: X^{m+m^{\prime}} \rightarrow X^{n+n^{\prime}}$ is defined as usual, as are the permutations. With these structures, $X$ is a PACT. A PACT $\mathbf{P}$ is said to act on the complex $X$ if there is given a morphism $\mathbf{P} \rightarrow \mathbf{X}$ of PACTs.

Now some examples. Just as each multiplicative group $\Pi$ yields a group algebra $K(\Pi)$, so each PROP H yields a PACT $K(\mathbf{H})$ with trivial grading and differential; define each

$$
K(\mathrm{H})\left(\begin{array}{l}
m \\
n
\end{array}\right)
$$

to be the free $K$-module generated by the elements of

$$
\mathbf{H}\left(\begin{array}{c}
m \\
n
\end{array}\right) \text {. }
$$

To describe hopf algebras, take the free PACT with generators $p: 2 \rightarrow 1, u: 0 \rightarrow 1, q: 1 \rightarrow 2$, and $v: 1 \rightarrow 0$, and impose the relations (24.5) for an algebra, the relations 
$(q \otimes 1) q=(1 \otimes q) q, \quad(v \otimes 1) q=1=q(1 \otimes v) \quad$ (for a coalgebra),

the relations which would state that $u$ (respectively, $v$ ) is a map of coalgebras (respectively, algebras),

$$
v p=v \otimes v: 2 \rightarrow 0, q u=u \otimes u: 0 \rightarrow 2, v u=1: 0 \rightarrow 0,
$$

and, finally, the relation corresponding to the diagram (17.4),

$$
q p=(p \otimes p)(1 \otimes \tau \otimes 1)(q \otimes q): 2 \rightarrow 2 .
$$

These determine a PROP $\mathbf{H}_{f}$. Form the PACT $K\left(\mathbf{H}_{f}\right)$. The statement that $K\left(\mathbf{H}_{f}\right)$ acts on $X$ is exactly the statement that $X$ is a DG( $K$-Mod)-hopf algebra, because the action on $X$ does give the product $p$, the unit $u$, the coproduct $q$, and the co-unit $v$ for $X$, satisfying the requisite identities. If we add the relation $p \tau=p$, we get the PACT $K\left(\mathrm{H}_{f c}\right)$ for commutative hopf algebras; then adding $\tau q=q$ gives the PACT $K\left(\mathrm{H}_{f c c}\right)$ for those hopf algebras with product and coproduct both commutative.

We already know ( $\$ 23)$ that each commutative DG-hopf algebra $U$ yields a commutative DG-hopf algebra $\bar{B}^{\bullet}(U)$-in other words, each action of $K\left(\mathbf{H}_{f c}\right)$ on $U$ determines an action on $\bar{B}^{\bullet}(U)$. Making extensive use of the DG-comparison homotopy, one may prove

THEOREM 25.1. If $K\left(\mathrm{H}_{f c c}\right)$ acts on $U$, then there is a PACT $\mathbf{P} \supset K\left(\mathbf{H}_{f c}\right)$ which acts on $B^{\cdot}(U)$ and on $\bar{B}^{\bullet}(U)$ and a map $\theta: \mathrm{P} \rightarrow K\left(\mathrm{H}_{f c c}\right)$ of PACTs such that the induced homology map

$$
\theta_{*}: H(\mathrm{P}) \cong H\left(K\left(\mathrm{H}_{f c c}\right)\right)
$$

is an isomorphism.

This is a covert statement of the existence of higher homotopies. For example, the generator $q$ acting on $\bar{B}^{\bullet}$ gives a coproduct which is not commutative, but gives a 0 -cycle $\tau q-q$ with image 0 under $\theta$. By the asserted isomorphism $\theta_{*}, \mathbf{P}$ must then contain a morphism $q_{1}: 1 \rightarrow 2$ with $D q_{1}=\tau q-q$ and, similarly, morphisms $q_{k}: 1 \rightarrow 2$ with $D q_{k+1}=\tau q_{k}-q_{k}$. These are the higher homotopies usually used to construct Steenrod squares. Similarly, corresponding to the relation (1), there is a morphism $r: 2 \rightarrow 2$ in $\mathrm{P}$ with

$$
D r=q_{1} p-(p \otimes p)\left(\tau q_{1} \otimes q_{0}+q_{0} \otimes q_{1}\right)
$$

-and a vast maze of such higher homotopies. Given this $\mathbf{P}$ acting on $U$, we conjecture that $\mathbf{P}$ will also act on $\bar{B}^{\circ}(U)$ in such a way that the action will be consistent with the passage from the iterated bar construction to the spaces $K(\Pi, n)$. 
26. Bicategories. The tensored categories, the PROPs, and the PACTs all have the following form: a triple $(\mathrm{B}, \otimes, K)$ consisting of a category $\mathbf{B}$, with a multiplication $\otimes$ and a ground object $K$, together with jointly coherent natural isomorphisms $a$ (associativity of $\otimes), c$ (commutativity of $\otimes$ ), and $e, e^{\prime}(K$ a left and right identity for $\otimes$ ). (In the case of PROPs and PACTs, $a, e$, and $e^{\prime}$ are the identity, while $c$ gives the transposition $\tau \in S(2)$ and hence, via coherence, the whole permutation structure.) Call such a triple $(\mathbf{B}, \otimes, K)$, or $\mathbf{B}_{\otimes}$ for short, a bicategory. While a category exhibits the functor hom, a bicategory exhibits both the basic functors hom and $\otimes$ for homological algebra. The bicategories have additional uses; for instance, we may describe the categories $\mathbf{C}$ "with morphisms in a given bicategory $\mathbf{B}_{\otimes}$ " as those categories $\mathbf{C}$ for which each hom $\mathrm{C}\left(C, C^{\prime}\right)$ is an object of B while composition in $\mathbf{C}$ is a morphism hom $\left(C^{\prime}, C^{\prime \prime}\right) \otimes \operatorname{hom}\left(C, C^{\prime}\right)$ $\rightarrow \operatorname{hom}\left(C, C^{\prime \prime}\right)$ of B. The desirability of such a description has long been recognized: For example, when $\mathbf{B}_{\otimes}$ is the category of graded sets with $\otimes$ the product functor, a $\mathbf{B}_{\otimes}$-category is a graded category; when $\mathbf{B}_{\otimes}$ is the category of abelian groups with the usual tensor product, a $\mathbf{B}_{\otimes}$-category is a (pre)-additive category (an additive category, less the biproduct axiom); when $\mathbf{B}_{\otimes}$ is DG( $K$-Mod), $\mathbf{C}$ is a differential category, as employed in the unpublished work of Eilenberg-Moore.

Another closely related notion is that of a category with an internal homfunctor (Kelly [59]) or an autonomous category (Linton [71]). Such a category $\mathbf{K}$ has a faithful functor $\mathbf{K} \rightarrow$ Ens, written $A \rightarrow|A|$, a functor Hom: $\mathbf{K}^{\mathrm{p}} \times \mathbf{K} \rightarrow \mathbf{K}$ and a natural equivalence of the composite $|\mathrm{Hom}|$ to the ordinary (set-valued) hom functor. Moreover, if $H_{A}$ is the internal covariant hom functor, with $H_{A}(B)$ $=\operatorname{Hom}(A, B)$, there is to be a functor $T_{A}: \mathbf{K} \rightarrow \mathbf{K}$ and a natural isomorphism

$$
\operatorname{Hom}\left(T_{A}(B), C\right) \cong \operatorname{Hom}\left(B, H_{A}(C)\right) ;
$$

in other words, $T_{A}$ is a strong adjoint to $H_{A}$. Finally, there is a natural isomorphism $H_{A} H_{B} \cong H_{B} H_{A}$ compatible, under $h_{A}=\left|H_{A}\right|$, with the familiar bijection $h_{|A|} h_{|B|} \cong h_{|B|} h_{|A|}$. This last condition insures that $T_{A}(B)$ can be regarded as a multiplication $A \otimes B$, and insures coherent associativity and commutativity. Such autonomous categories promise to be useful, in the same way in which the present notions of categories are effective tools in getting a systematic arrangement and understanding of quite a variety of mathematical ideas.

\section{BIBLIOGRAPHY}

This contains recent references on categories. Additional earlier references are to be found in the bibliography of MacLane [77] under the following names and dates: 
Amitsur (1961), Atiyah (1956), Buchsbaum (1955, 1959, 1960), Butler and Horrocks (1961), Ehresmann (1957), Eilenberg-MacLane (1945), Eilenberg-Moore (1962), Freyd (1960), Heller (1958), Hilton-Ledermann (1958), Hilton-Rees (1961), Huber (1961), Kleisli (1962), Lubkin (1960), MacLane (1961), Puppe (1962), Röhrl (1962), Watts (1960), Yoneda (1960).

1. J. Bénabou, Catégories avec multiplication, C. R. Acad. Sci. Paris 256 (1963), 1887-1890.

2. - Algebre elementaire dans les categories avec multiplication, C. R. Acad. Sci. Paris 258 (1964), 771-774.

3. G. H. Berman, Functors in the category of locally complex spaces, Soviet Math. Dokl. 5 (1964), 99-101.

4. I. E. Burmistrowitsch, Embedding of an additive category in a category with direct products, Soviet Math. Dokl. 1 (1960), 742-744.

5. M. S. Calenko, On the foundations of the theory of categories, Uspehi Mat. Nauk 15 (1960), No. 6, 53-58 (Russian); English transl., Russian Math. Surveys 15 (1960), No. 6, 47-51.

6. - Regular joins and special subdirect sums in categories, Mat. Sb. (N.S.) 57 (1962), 75-94. (Russian)

7. - Completion of categories by free and direct joins of objects, Mat. Sb. (N.S.) 60 (1963), 235-256. (Russian)

8. - Correspondences over a quasi-exact category, Soviet Math. Dokl. 5 (1964), 416-418.

9. P. M. Cohn, Universal algebra, Harper, New York, 1965.

10. P. Dedecker and J. Mersch, Précatégories et relations d'equivalence dans les catégories, C. R. Acad. Sci. Paris 256 (1963), 4811-4814.

11. S. E. Dickson, $A$ torsion theory for abelian categories (to appear).

12. A. Dold, Ueber die Steenrodschen Kohomologieoperationen, Ann. of Math. 73 (1961), 258-294.

13. - Lectures on homotopy theory and half exact functors. Notes taken and prepared by F. Oört, Mathematisches Institut, Amsterdam, 1964.

14. B. Eckmann and P. J. Hilton, Homotopy groups of maps and exact sequences, Comment. Math. Helv. 34 (1960), 271-304. 1-21.

15. - Operators and cooperators in homotopy theory, Math. Ann. 141 (1960),

16. - Structure maps in group theory, Fund. Math. 50 (1961/62), 207-221.

17. - Group-like structures in general categories. I, Math. Ann. 145 (1961/62), $227-255$.

18. - Group-like structures in general categories. II. Equalizers, limits, lengths, Math. Ann. 151 (1963), 150-186.

19. - Group-like structures in general categories. III. Primitive categories, Math. Ann. 150 (1963), 165-187.

20. Ch. Ehresmann, Catégories topologiques et catégories différentiables, Colloque de geometrie différentielle globale, Bruxelles, 1958.

21. - Catégories inductives et pseudogroupes, Ann. Inst. Fourier (Grenoble) 10 (1960), 307-332.

22. - Categorie des foncteurs types, Rev. Un. Mat. Argentina 20 (1960), 194-209.

23. — Catégories doublées et catégories structurées, C. R. Acad. Sci. Paris 256 (1963), 1198-1201. 
24. - Catégories structurées d'operateurs, C. R. Acad. Sci. Paris 256 (1963), 2080-2083.

25. - Sous-structures et applications KK-covariantes, C. R. Acad. Sci. Paris 256 (1963), 2280-2283.

26. — , Product croisé des catégories, C. R. Acad. Sci. Paris 258 (1964), 24612464.

27. - Structures quotients, Comment. Math. Helv. 38 (1964), 219-283.

28. - Catégories structurées, Ann. Sci. Ecole Norm. Sup. 80 (1963), 349426.

29. - Sous-structures et catégories ordonnées, Fund. Math. 54 (1964), 211228.

30. S. Eilenberg and S. MacLane, Natural isomorphisms in group theory, Proc. Nat. Acad. Sci. U.S.A. 28 (1942), 537-543.

31. D. B. A. Epstein, Steenrod operations in abelian categories (to appear).

32. I. Fleischer, Sur le probleme d'application universelle de M. Bourbaki, C. R. Acad. Sci. Paris 254 (1962), 3161-3163.

33. P. Freyd, Relative homological algebra made absolute, Proc. Nat. Acad. Sci. U.S.A. 49 (1963), 19-20.

34. - Abelian categories, Harper and Row, New York, 1964.

35. D. B. Fuks, On duality in homotopy theory, Soviet Math. Dokl. 2 (1961), 15751578.

36. - Natural transformations of functors in the category of topological spaces, Mat. Sb. (N.S.) 62 (1963), 160-179. (Russian)

37. D. B. Fuks and A. B. Švarc, On the homotopy theory of functors in the category of topological spaces, Soviet Math. Dokl. 3 (1962), 444-447.

38. I. M. Gelfand and G. E. Silov, Categories of finite-dimensional spaces, Vestnik Moskov Univ. Ser. I Mat. Meh. 1963, no. 4, 27-48. (Russian, German summary.)

39. R. Godement, Théorie des faisceaux, Hermann, Paris, 1958.

40. J. M. Gray, Sheaves with values in a category, Topology (to appear).

41. A. Grothendieck, Sur quelques points d'algèbre homologique, Tôhoku Math. J. 9 (1957), 119-221.

42. - Technique de descente et thêoremes d'existence en gêométrie algêbrique. II, Séminaire Bourbaki 12 (1959/1960), exp. 195, Secrétariat Mathématique, Paris, 1961.

43. - Technique de construction en géométrie analytique. IV. Formalisme général des foncteurs représentables, Séminaire H. Cartan 13 (1960/1961), exp. 11, Secrétariat Mathématique, Paris, 1962.

44. A. Grothendieck and J. Dieudonné, Eléments de géométrie algébrique, Vol. III, Publ. Math. Inst. des Hautes Etudes 11 (1961), 1-167.

45. A. Ja. Helemskii, On algebras of nilpotent operators and categories associated with them, Vestnik Moskov Univ. Ser. I. Mat. Meh. 1963, no. 4, 49-55. (Russian, English summary)

46. P. J. Higgins, Presentations of groupoids, with applications to groups, Proc. Cambridge Philos. Soc. 60 (1964), 7-20.

47. P. J. Hilton, Note on free and direct products in general categories, Bull. Soc. Math. Belg. 13 (1961), 38-49.

48. P. J. Hilton and W. Ledermann, On the Jordan-Hölder theorem in homological monoids, Proc. London Math. Soc. 10 (1960), 321-334.

49. H. J. Hoehnke, Zur Theorie der Gruppoide. I, II, Math. Nachr. 24 (1962), 137168, 169-179; III, Acta Math. Acad. Sci. Hungar. 13 (1962), 91-100; IV, V, Monatsb. Deutsche Akad. Wiss. Berlin 4 (1962), 337-342, 539-544. 
50. - Einige Bemerkungen zur Einbettbarkeit von Kategorien in Gruppen, Math. Nachr. 25 (1963), 179-190.

51. F. Hofmann, Ueber eine die Kategorie der Gruppen umfassende Kategorie, S.-B. Bayer. Akad. Wiss. Math.-Nat. Kl. (1960), 163-204.

52. P. J. Huber, Standard constructions in abelian categories, Math. Ann. 146 (1962), 321-325.

53. M. Hušek, S-categories, Comment. Math. Univ. Carolinae 5 (1964), 37-46.

54. J. R. Isbell, Natural sums and direct decompositions, Duke Math. J. 27 (1960), $507-512$.

55. - Subobjects, adequacy, completeness, and categories of algebras, Rozprawy Mat. 36 (1964), 3-32. 49.

56. - Two set-theoretical theorems in categories, Fund. Math. 53 (1963), 43-

57. J. P. Jans, Rings and homology, Holt, Rinehart and Winston, New York, 1964.

58. D. M. Kan, Adjoint functors, Trans. Amer. Math. Soc. 87 (1958), 294-329.

59. G. M. Kelly, Tensor products in categories. I, J. Algebra (to appear).

60. - Complete functors in homology. I, II, Proc. Cambridge Philos. Soc. 60 (1964), 721-735; 737-749.

61. - On MacLane's conditions for coherence of natural associativities, commutativities, etc., J. Algebra (to appear).

62. J. F. Kennison, Reflective functors in general topology and elsewhere, Trans. Amer. Math. Soc. (to appear).

63. H.-J. Kowalsky, Kategorien topologischer Räume, Math. Z. 77 (1961), 249-272.

64. A. G. Kuroš, Direct decomposition in algebraic categories, Trudy Moskov Mat. Obšč. 8 (1959), 319-421; Amer. Math. Soc. Transl. (2) 27 (1963), 231-255.

65. A. G. Kuros, A. H. Livšic and E. G. Sulgelfer, Foundations of the theory of categories, Uspehi Mat. Nauk 15 (1960), No. 6, 3-52 (Russian), Russian Math. Surveys 15 (1960), 1-45 (English transl.), Zur Theorie der Kategorien, VEB Deutscher Verlag der Wissenschaften, Berlin, 1963.

66. F. W. Lawvere, Functorial semantics of algebraic theories, Dissertation, Columbia University, New York, 1963.

67. - Functorial semantics of algebraic theories, Proc. Nat. Acad. Sci. U.S.A. 50 (1963), 869-872.

68. J. B. Leicht, On commutative squares, Canad. J. Math. 15 (1963), 59-79.

69. - Remarks on axiomatic theory of relations (to appear).

70. F. E. J. Linton, The functorial foundations of measure theory, Dissertation, Columbia University, New York, 1963.

71. - Autonomous categories and duality, J. Algebra (to appear).

72. A. H. Livšic, Direct decompositions in algebraic categories, Trudy Moskov. Mat. Obšč. 9 (1960), 129-141. (Russian)

73. - Direct decompositions with indecomposable components in algebraic categories, Mat. Sb. (N.S.) 51 (1960), 427-458. (Russian)

74. - The addition of mappings and the concept of center in categories, Mat. Sb. (N.S.) 60 (1963), 159-184. (Russian)

75. Saunders MacLane, Duality for groups, Bull. Amer. Math. Soc. 56 (1950), 485-516.

76. - Locally small categories and the foundations of set theory. Infinitistic Methods, Oxford, 1961; pp. 25-43.

77. —- Homology, Springer, Berlin; Academic Press, New York, 1963.

78. - Natural associativity and commutativity, Rice University Studies 49 (1963), No. 4, 28-46. 
79. J. Milnor and J. C. Moore, On the structures of hopf algebras (to appear).

80. B. Mitchell, The full embedding theorem, Amer. J. Math. 86 (1964), 619-637.

81. B. S. Mitjagin and A. S. Svarc, Functors in categories of Banach spaces, Uspehi Mat. Nauk 19 (1964), 65-130. (Russian)

82. K. Morita, Category isomorphisms and endomorphism rings of modules, Trans. Amer. Math. Soc. 103 (1962), 451-469.

83. G. Nöbeling, Ueber die Derivierten des Inversen und des dirketen Limes einer Modulfamilie, Topology 1 (1962), 47-61.

84. Yvon Nouazé, Catégories localement de type fini et catégories localement noethériennes, C. R. Acad. Sci. Paris 257 (1963), 823-824.

85. T. Ohkuna, Duality in mathematical structure, Proc. Japan Acad. 34 (1958), 6-10.

86. F. Oört, Natural maps of extension functors, Nederl. Akad. Wetensch. Ser. A 66, No. $4=$ Indag. Math. 25 (1963), 559-566. 235.

87. — , Yoneda extensions in abelian categories, Math. Ann. 153 (1964), 227-

88. J. E. Roos, Sur les foncteurs dérivées de lim๘. Applications, C. R. Acad. Sci. Paris 252 (1961), 3702-3704.

89. P. Samuel, On universal mappings and free topological groups, Bull. Amer. Math. Soc. 54 (1948), 591-598.

90. J. A. Schafer, The dual of the flabby is the bar, Proc. Amer. Math. Soc. (to appear).

91. S. K. Seghal, Ringoids with minimum conditions, Math. Z. 83 (1964), 395-408.

92. Z. Semadeni, Free and direct objects, Bull. Amer. Math. Soc. 69 (1963), 63-65.

93. - Projectivity, injectivity, and duality, Rozprawy Mat. 35 (1963), 1-47.

94. W. Shih, Ensembles simpliciaux et operations cohomologiques, Séminaire H. Car$\tan (1958 / 1959)$, exp. 7, Secrétariat Mathématique, Paris.

95. J. Sonner, On the formal definition of categories, Math. Z. 80 (1962), 16.3-176.

96. —_, Universal and special problems, Math. Z. 82 (1963), 200-211.

97. J. D. Stasheff, Homotopy associativity of $H$-spaces. I, Trans. Amer. Math. Soc. 108 (1963), 275-292.

98. E. G. Sulgeffer, On the general theory of radicals in categories, Mat. Sb. (N.S.) 51 (1960), 487-500. (Russian)

99. - On the lattice of ideals in an object of a category. I, II, Mat. Sb. (N.S.) 54 (1961), 209-224, ibid. 62 (1963), 335-344. (Russian)

100. - Tame embeddings of categories. Mat. Sb. (N.S.) 61 (1963), 467-503.

101. A. S. Švarc, Duality of functors, Soviet Math. Dokl. 4 (1963), 89-92.

102. - Functors in the categories of Banach spaces, Soviet Math. Dokl. 4 (1963), 317-320.

103. R. G. Swan, Theory of sheaves, Univ. Chicago Press, Chicago, Ill., 1964.

104. C. L. Walker and E. A. Walker, Quotient categories and rings of quotients (to appear).

105. C. E. Watts, Homological algebra of categories. I. (to appear).

106. J. A. Zilber, Categories in homotopy theory, Dissertation, Harvard University, Cambridge, Mass., 1963.

The University of Chicago 